\title{
MUSE integral-field spectroscopy towards the Frontier Fields cluster Abell S1063
}

\section{Properties of low luminosity Lyman $\alpha$ emitters at $z>3$}

\author{
W. Karman ${ }^{1}$, K. I. Caputi ${ }^{1}$, G. B. Caminha ${ }^{2}$, M. Gronke ${ }^{3}$, C. Grillo, ${ }^{4,5}$, I. Balestra ${ }^{6,7}$, P. Rosati ${ }^{2}$, E. Vanzella ${ }^{8}$, D. Coe ${ }^{9}$, \\ M. Dijkstra ${ }^{3}$, A. M. Koekemoer ${ }^{9}$, D. McLeod ${ }^{10}$, A. Mercurio ${ }^{11}$, and M. Nonino ${ }^{7}$
}

\author{
${ }^{1}$ Kapteyn Astronomical Institute, University of Groningen, Postbus 800, 9700 AV Groningen, The Netherlands \\ e-mail: karman@astro.rug.nl \\ 2 Dipartimento di Fisica e Scienze della Terra, Università degli Studi di Ferrara, via Saragat 1, 44122 Ferrara, Italy \\ 3 Institute of Theoretical Astrophysics, University of Oslo, Postboks 1029 Blindern, 0315 Oslo, Norway \\ 4 Dark Cosmology Centre, Niels Bohr Institute, University of Copenhagen, Juliane Maries Vej 30, 2100 Copenhagen, Denmark \\ 5 Dipartimento di Fisica, Università degli Studi di Milano, via Celoria 16, 20133 Milano, Italy \\ ${ }^{6}$ University Observatory Munich, Scheinerstrasse 1, 81679 Munich, Germany \\ INAF-Osservatorio Astronomico di Trieste, via G. B. Tiepolo 11, 34143 Trieste, Italy \\ INAF-Bologna Astronomical Observatory, via Ranzani 1, 40127 Bologna, Italy \\ Space Telescope Science Institute, 3700 San Martin Drive, Baltimore, MD 21208, USA \\ ${ }_{10}$ SUPA, Institute for Astronomy, University of Edinburgh, Royal Observatory, Edinburgh EH9 3HJ, UK \\ 11 INAF-Osservatorio Astronomico di Capodimonte, via Moiariello 16, 80131 Napoli, Italy
}

Received 3 June 2016 / Accepted 23 August 2016

\begin{abstract}
In spite of their conjectured importance for the Epoch of Reionization, the properties of low-mass galaxies are currently still very much under debate. In this article, we study the stellar and gaseous properties of faint, low-mass galaxies at $z>3$. We observed the Frontier Fields cluster Abell S1063 with MUSE over a $2 \operatorname{arcmin}^{2}$ field, and combined integral-field spectroscopy with gravitational lensing to perform a blind search for intrinsically faint Ly $\alpha$ emitters (LAEs). We determined in total the redshift of 172 galaxies of which 14 are lensed LAEs at $z=3-6.1$. We increased the number of spectroscopically-confirmed multiple-image families from 6 to 17 and updated our gravitational-lensing model accordingly. The lensing-corrected Ly $\alpha$ luminosities are with $L_{\mathrm{Ly} \alpha} \lesssim 10^{41.5} \mathrm{erg} / \mathrm{s}$ among the lowest for spectroscopically confirmed LAEs at any redshift. We used expanding gaseous shell models to fit the Ly $\alpha$ line profile, and find low column densities and expansion velocities. This is, to our knowledge, the first time that gaseous properties of such faint galaxies at $z \gtrsim 3$ are reported. We performed SED modelling to broadband photometry from the $U$ band through the infrared to determine the stellar properties of these LAEs. The stellar masses are very low $\left(10^{6-8} M_{\odot}\right)$, and are accompanied by very young ages of 1-100 Myr. The very high specific star-formation rates $\left(\sim 100 \mathrm{Gyr}^{-1}\right)$ are characteristic of starburst galaxies, and we find that most galaxies will double their stellar mass in $\$ 20$ Myr. The UV-continuum slopes $\beta$ are low in our sample, with $\beta<-2$ for all galaxies with $M_{\star}<10^{8} M_{\odot}$. We conclude that our low-mass galaxies at $3<z<6$ are forming stars at higher rates when correcting for stellar mass effects than seen locally or in more massive galaxies. The young stellar populations with high star-formation rates and low H I column densities lead to continuum slopes and LyC-escape fractions expected for a scenario where low mass galaxies reionise the Universe.
\end{abstract}

Key words. galaxies: high-redshift - galaxies: distances and redshifts - galaxies: clusters: individual: Abell S1063 gravitational lensing: strong - galaxies: evolution - techniques: imaging spectroscopy

\section{Introduction}

The evolution of the brightest galaxies in the Universe has now been studied in significant detail out to $z \sim 8$ (e.g Bouwens et al. 2014; Salmon et al. 2015; Caputi et al. 2015), and is in accordance with the now well-established $\Lambda \mathrm{CDM}$ model. The study of low-mass, faint galaxies at high- $z$ is, instead, almost a completely unknown territory. Gaining a greater knowledge of these faint galaxies is important as they are the building blocks of the observed more massive galaxies at lower redshifts, and they are currently seen as the main candidates for reionizing the Universe at $z=6-10$ (Wise et al. 2014; Kimm \& Cen 2014, but see Sharma et al. 2016).

Observationally, high-redshift, low-mass galaxies have been elusive to date. The Lyman break technique (e.g.
Steidel et al. 1996, 2003; Bouwens et al. 2011) and spectralenergy-distribution (SED) fitting codes (e.g. Caputi et al. 2011; Ilbert et al. 2013), which are well proven for intermediate-mass and massive galaxies, are not applicable to these faint sources in all but the deepest multiwavelength studies (e.g. Ouchi et al. 2010; Schenker et al. 2013) or until The James Webb Space Telescope is operating (e.g. Gardner et al. 2009; Bisigello et al. 2016). Therefore, other approaches are needed to understand the faint end of the galaxy population. One possible approach is looking for counterparts of absorbers in quasar lines-ofsights (e.g. Arrigoni Battaia et al. 2016), but this is only feasible for bright quasars with long spectroscopic observations (e.g Rauch et al. 2008). Fortunately, the Ly $\alpha$ line is redshifted in the optical domain for galaxies at $z \gtrsim 3$. Although stars in massive galaxies are often surrounded by a dusty inter-stellar 
and circum-galactic medium which absorbs all Ly $\alpha$ photons (e.g. Laursen et al. 2009), less-massive star-forming galaxies are often found with significant Ly $\alpha$ emission (e.g. Oyarzún et al. 2016). Therefore, searching for galaxies with strong emission lines in the optical can be used to identify low-mass high-redshift galaxies.

Another possibility are optical narrowband studies, which search for galaxies with strong emission lines (e.g. Nilsson et al. 2009; Nakajima et al. 2012; Matthee et al. 2016) by looking for sources with strong colours between the narrowbands and overlapping broadband observations. By applying additional colour cuts representative of high-redshift galaxies, reliable candidates for Ly $\alpha$ emitters (LAEs) can be found. However, it has been shown that low-redshift extreme line-emitters can contaminate this sample (e.g. Atek et al. 2011; Pénin et al. 2015), and galaxies with intermediate $\operatorname{Ly} \alpha$ line strengths will not survive the colour cuts. Another disadvantage of using narrow-band studies is that these selections are only useful for very narrow redshift ranges.

Although Ly $\alpha$ has become the most important line to identify galaxies with redshifts between $2.5<z<7$ (e.g. Shimasaku et al. 2006; Dawson et al. 2007; Díaz et al. 2015; Trainor et al. 2015), it is still unclear what governs whether a galaxy is a LAE or not. It has been found that LAEs are in general less dusty than LBGs (e.g. Atek et al. 2014), but they have very similar stellar properties at fixed luminosity (Shapley et al. 2001; Kornei et al. 2010; Yuma et al. 2010; Mallery et al. 2012; Jiang et al. 2016). There is evidence however, that the prevalence of Ly $\alpha$ emission is much higher in less luminous systems (Stark et al. 2010; Forero-Romero et al. 2012) and less massive systems (Oyarzún et al. 2016). A similar trend is found for the equivalent width (EW) of $\operatorname{Ly} \alpha$, which anticorrelates with UV luminosity (e.g. Shapley et al. 2003; Gronwall et al. 2007; Kornei et al. 2010). Further, the fraction of LBGs with $\mathrm{Ly} \alpha$ emission increases with redshift out to $z \sim 6$ (e.g. Ouchi et al. 2008; Cassata et al. 2011, 2015; Pentericci et al. 2011; Curtis-Lake et al. 2012; Schenker et al. 2012; Henry et al. 2012), but experiences a rapid decrease afterwards (e.g. Kashikawa et al. 2011; Caruana et al. 2012, 2014; Ono et al. 2012; Schenker et al. 2012; Stark et al. 2010; Pentericci et al. 2014). This drop has theoretically only been explained succesfully as arising from reionization (Dijkstra et al. 2011; Jensen et al. 2013; Mesinger et al. 2015; Choudhury et al. 2015), although additional processes might be involved (e.g. Dijkstra 2014; Choudhury et al. 2015).

While the broadband photometry can reveal much about the stellar and dust properties of galaxies, the Ly $\alpha$ line profile provides important information on the properties of the gas (e.g. Verhamme et al. 2006, 2008; Sawicki et al. 2008). Since only Ly $\alpha$ photons shifted out of resonance can effectively escape the galaxy, moving gas clouds such as outflows allow Ly $\alpha$ photons to escape (e.g. Schaerer et al. 2011; Laursen et al. 2013; Dijkstra 2014). Dust absorbs the Ly $\alpha$ photons and emits them at longer wavelengths, while a patchy distribution of the surrounding medium allows the photons to escape. Therefore, by careful modelling of the $\operatorname{Ly} \alpha$ line, one can learn about the properties of the gaseous medium in and surrounding galaxies. Recently, it has been demonstrated that galaxies with extreme optical and near-UV emission lines are often exhibiting narrow Ly $\alpha$ emission (Cowie et al. 2011; Henry et al. 2015; Izotov et al. 2016; de Barros et al. 2016; Vanzella et al. 2016a). The fact that these galaxies are found to have $\operatorname{Ly} \alpha$ emission both at low and high redshift, indicates that these so-called "Green Peas" might be good analogues of the high-redshift
LAEs (e.g. Amorín et al. 2010, 2015). Another indication for a close resemblance between these galaxies is the finding that low stellar mass, high SFR, and low dust content correlate with Ly $\alpha$ emission both at low (e.g. Cowie et al. 2011; Henry et al. 2015) and high redshift (e.g. Jiang et al. 2016). In addition, Vanzella et al. (2016b) and Izotov et al. (2016) found Lyman continuum leakage for two of these galaxies, making them important candidates for reionization.

The Frontier Fields programme (hereafter FF, PI: J. Lotz; see Lotz et al. 2016; and Koekemoer et al. 2016) provides an excellent opportunity to study intrinsically faint galaxies at high redshifts. Massive galaxy clusters provide a boost in depth thanks to the effect of gravitational lensing. The deep HST coverage over 7 different bands provides photometry for intrinsically faint sources which allows us to study their properties. Combining this deep gravitionally-lensed photometric survey with spectroscopy allows us to determine accurate stellar and gaseous properties down to an intrinsic faintness which is otherwise currently unachievable within a reasonable observing time. Abell S1063 (AS1063), the cluster studied here, is among the best studied FF clusters for which we have one of the best constrained and most precise strong lensing model available so far (e.g. Monna et al. 2014; Johnson et al. 2014; Richard et al. 2014; Caminha et al. 2016b; Diego et al. 2016).

In Karman et al. (2015, hereafter Paper I), we showed that using gravitational lensing in combination with the integral field spectrograph Multi Unit Spectroscopic Explorer (MUSE) we have been able to identify previously undetected, intrinsically faint LAE. In this work we expand on our previous results by adding observations of a second MUSE pointing covering the second half of the cluster, and using Ly $\alpha$ line profile modelling in combination with broadband photometry to study the properties of LAEs at $3<z<6$. In addition, we present an updated redshift catalogue using the full MUSE dataset.

The layout of this paper is as follows. In Sect. 2 we give a brief overview of the MUSE performance and the obtained data, followed by the data reduction process. In Sect. 3, we describe our spectroscopic results, including the determined redshifts and emission line properties. We used spectral energy distribution (SED) fitting to the broadband photometry to study the stellar properties of these objects in Sect. 4. We summarise and discuss our findings in Sect. 5, and present our conclusions in Sect. 6. Throughout this paper, we adopt a cosmology with $H_{0}=70 \mathrm{~km} \mathrm{~s}^{-1} \mathrm{Mpc}^{-1}, \Omega_{\mathrm{M}}=0.3$, and $\Omega_{\Lambda}=0.7$. Unless we specify otherwise, all given star formation rates (SFRs) are derived from spectral energy distribution (SED) modelling. All magnitudes refer to the $\mathrm{AB}$ system, and we use a Chabrier initial mass function (IMF) over stellar masses in the range $0.1-100 M_{\odot}$.

\section{Observations}

\subsection{Photometry}

The Hubble Frontier Fields programme ${ }^{1}$ (FF, PI: J. Lotz; see Lotz et al. 2016; and Koekemoer et al. 2016) targets six galaxy clusters with large magnification factors, among which is AS1063. The programme targets each cluster for a total of 140 orbits, divided over 7 bands in the optical and near infrared (NIR), reaching a $5 \sigma$ depth of $\sim 29$ mag in each of these bands. We used the available public HST data from this programme, retrieved from the Frontier Fields page at the STScI MAST

\footnotetext{
https://archive.stsci. edu/prepds/frontier/
} 
Archive, to detect sources and measure locations and magnitudes of sources, adopting the current zeropoints, provided by the ACS and WFC3 teams at STScI, which are tabulated on the same MAST Archive page for these specific FF filters. At the time of writing, the optical bands were fully observed for AS1063, while the NIR observations have had only a single orbit exposure. We used the v0.5 data products, which do not contain self calibration for this cluster. We used the images with a spatial resolution of $0.060^{\prime \prime}$, in order to have a uniform pixel scale, without oversampling the NIR images.

In addition to being a FF cluster, AS1063 is also part of the Cluster Lensing and Supernova Survey with Hubble (CLASH, Postman et al. 2012) survey, which targets 25 gravitationally lensing clusters with HST in 16 bands. We supplement our FF data with the CLASH data in 5 additional bands. These data are significantly less deep, but provide additional information for the brightest objects. For all these filters, which are in addition to those used in the FF programme, we adopt the current zeropoints provided by the ACS and WFC3 teams at $\mathrm{STScI}^{2,3}$.

As the LAEs discussed here all lie at $z>2.8$, the NIR images from HST do not cover the wavelength range above $4000 \AA$ restframe. Information at longer wavelengths is therefore crucial to better constrain older stellar populations. We collected Hawk-I data in order to complement our data at longer wavelengths. The Hawk-I images ${ }^{4}$ were retrieved from the ESO Archive ${ }^{5}$. The whole dataset includes 997 images obtained in September 2015. After dark and flat correction, a first sky subtraction was performed without source masking. Sources extracted from these background subtracted images were used to solve the astrometry, where we used Scamp (Bertin 2006) in combination with a catalogue from an ESO-WFI-Rc stacked image as reference. Using SWARP (Bertin et al. 2002) we created a coadded image, which was used to create a segmentation map. We masked all source pixels in the original frame using the single-frame astrometric solution and the segmentation map, and estimated a new background from the masked image. Finally, we subtracted the estimated background and created a new final coadded image, with a $3 \sigma$ depth of $25.9 \operatorname{mag}_{\mathrm{AB}}{ }^{6}$.

We extracted magnitudes from the optical and NIR images using SEXTRACTOR. As most of these images have irregular morphologies due to lensing, see Fig. 2, we adopt Kron-like apertures rather than spherical apertures. We constructed a detection image for the FF photometry by combining the $F 435$, $F 606$, and $F 814 W$ images, and required that each source is detected at more than $1 \sigma$ in more than eight connected pixels. For the CLASH images, we used the detection image provided by the CLASH collaboration as a detection image, due to a different spacing and resolution. We note that this might introduce an offset in the colours of the galaxies between CLASH and FF detections, but this effect will be small compared to the error bars obtained from the shallower CLASH observations. We tested the validity of using Kron-radii, different detection images resulting in possible colour differences due to our approach in Appendix C. We used 32 deblending sub-threshold levels, with a relative minimum contribution of $0.1 \%$. The background

\footnotetext{
2 ACS zeropoints: http://wWw.stsci.edu/hst/acs/analysis/ zeropoints

WFC3 zeropoints: http://www.stsci.edu/hst/wfc3/phot_ zp_lbn

4 ESO Programme 095.A-0533, PI Brammer.

5 http://archive.eso.org

6 After submission of this paper, Brammer et al. (2016) released a public version of the Hawk-I data. We performed a comparison of the data, and found a similar quality.
}

is calculated locally using the weight maps provided by the FF team. We checked each individual detection if it was contaminated by other closeby galaxies, and removed detections when dubious, however we note that some galaxies might still suffer from contamination due to inaccurate background estimates. We noted that visually detected sources remained undetected by SExtractor in the $F 814 W$ and Hawk-I Ks observations. We used more aggressive detection settings for these bands, and added the relevant detections to our catalogue. For the HST images, we compared the errors provided by SEXTRACTOR with those measured from the RMS images provided by the FF team. We found that multiplying the SEXTRACTOR errors by a factor of 1.4 reconciled the different methods.

We also measured photometry in the available Spitzer Infrared Array Camera (IRAC) imaging in channel $1(\lambda=3.6 \mu \mathrm{m})$ and channel $2(\lambda=4.5 \mu \mathrm{m})^{7}$, which we mosaiced. This imaging covers a depth of typically $\sim 24.9$ magnitudes at $5 \sigma$, although this is inhomogeneous across the imaging as a result of the increased crowding and intracluster light near the centre of the field of view. These depths are also subject to being able to extract reliable photometry via deconfusion techniques.

The photometry in this imaging was measured using the deconfusion code TPHOT $^{8}$. Briefly, the user provides the code with spatial and surface brightness information for a catalogue of objects as detected in the high-resolution imaging (in this case, the HST F160W imaging). The code convolves galaxy templates taken from the high-resolution image with a transfer kernel in order to create the corresponding template in the low-resolution image. The fluxes of these low-resolution templates are all fitted together, in order to produce a best fitting model of the lowresolution image. For further details, the reader is referred to Merlin et al. (2015).

With this approach, we found two clear IRAC detections among our LAE sample, and an additional six with $\sim 2-4 \sigma$ detections. For the remaining objects, we used the locally estimated depth of the image to set an upper limit at 3 times the depth of the observation to better constrain the restframe optical properties. A caveat to our approach is the issue of excessive crowding in the cluster centre, where some of the candidates are situated. For these objects, extracting reliable photometry was particularly challenging, even when additionally attempting to fit the background to account for the cluster light, but we found that given their relatively large uncertainties, they had little effect on our results.

\subsection{Integral field spectroscopy}

The MUSE instrument mounted on the VLT (Bacon et al. 2012) is a powerful tool to blindly look for LAEs behind clusters. Its relatively large field of view $\left(1 \operatorname{arcmin}^{2}\right)$, spectral range (4750-9350 $\AA$ ), relatively high spatial $\left(0.2^{\prime \prime}\right)$ and spectral $(\sim 3000)$ resolution, and stability allowed us to find LAEs down to an observed flux of $10^{-18} \mathrm{erg} / \mathrm{s} / \mathrm{cm}^{2}$ in a $1 \times 1^{\prime}$ field with only $4 \mathrm{~h}$ of exposure.

AS1063 was targeted with MUSE to search for high-redshift galaxies (Paper I) and simultaneously aid in constraining the lens properties, see Caminha et al. (2016b, hereafter C16) for a detailed description of the used lensing models. The data on the south-western half of AS1063 was described in Paper I and

\footnotetext{
7 PI Soifer, programme ID 10170.

8 TPHOT is publicly available for downloading from www . astrodeep. eu/t-phot/, see also Merlin et al. (2015).
} 

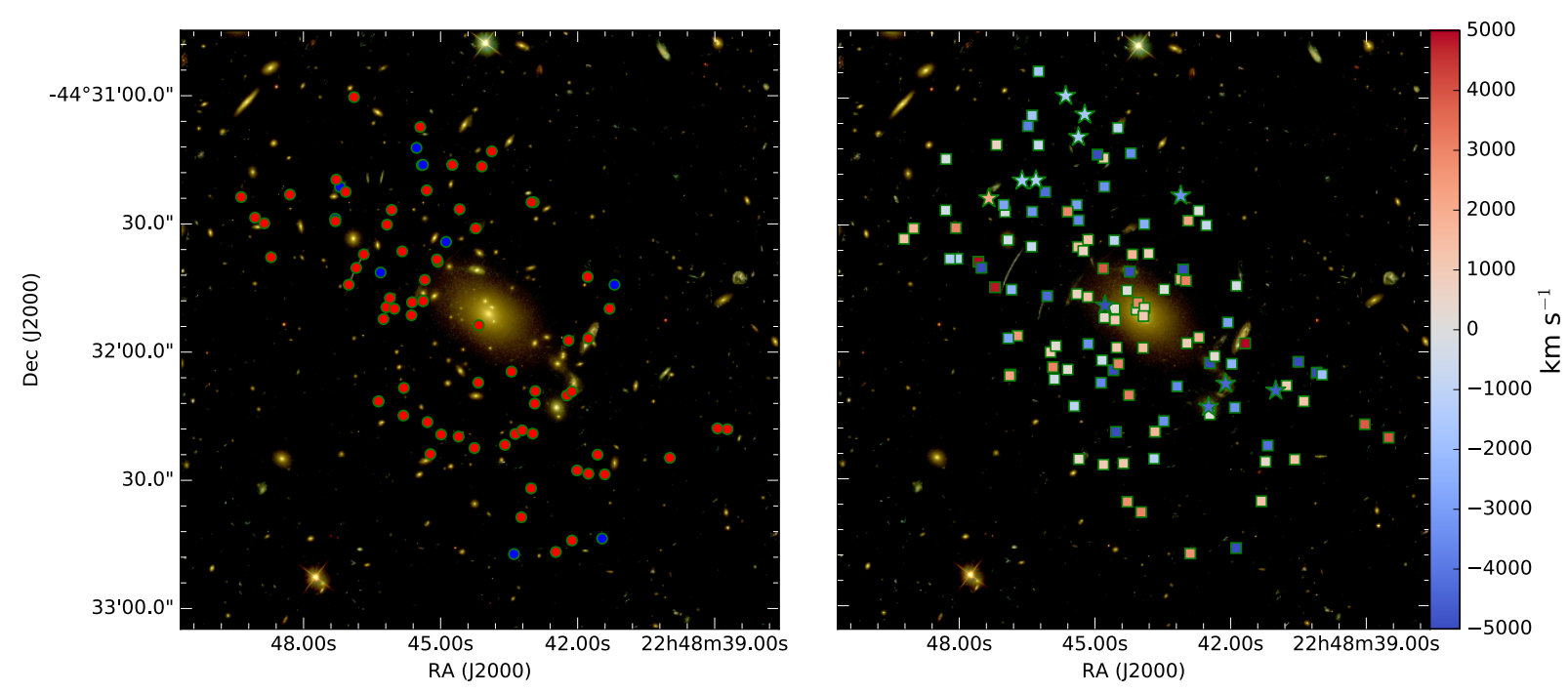

Fig. 1. Distribution map of the identified galaxies, in the fore and background (left) and in the cluster (right). The galaxies are overplotted on an HST-RGB image, consisting of the $F 435 W$ (blue), $F 606 W$ (green), and $F 814 W$ (red) filters from the FF programme. In the left panel, the background galaxies are shown with red circles, while foreground objects are shown with blue circles. In the right panel, squares correspond to passive cluster galaxies, while stars indicate active cluster galaxies, where their classification is based on the presence or absence of optical emission lines. The galaxies have been coloured according to their velocity relative to the cluster $(z=0.3475)$, with bluer colours meaning higher velocities towards us, and redder colours corresponding to higher velocities away from us, see also the colour bar on the right.

consists of 8 exposures of $1400 \mathrm{~s} \mathrm{each}^{9}$, or a total integration time of $3.1 \mathrm{~h}$. In this paper we add the north-eastern half of AS1063 to the available data, which has 12 exposures of $1440 \mathrm{~s}$, or a total integration time of $4.8 \mathrm{~h}^{10}$. We note that 4 of the later exposures were rated with a grade $\mathrm{C}$, meaning the observational requirements were not met. However, we did include these exposures to our datacube, as they did not decrease the spatial resolution, and did improve the depth of the final datacube.

Each pointing employed the same observing strategy, where we used observation blocks of 1440s which followed a dither pattern with offsets of a fraction of an arcsecond and rotations of 90 degrees to better remove cosmic rays and to obtain a better noise map. We followed the data reduction as described in Paper I for both pointings, and refer to that paper for details. Here we provide only a brief description of the data reduction. We used the standard pipeline of MUSE Data Reduction Software version 1.0 on all of the raw data. This pipeline includes the standard reduction steps like bias subtraction, flatfielding, wavelength calibration, illumination correction, and cosmic ray removal. We checked all wavelength calibrations for accuracy and verified the wavelength solutions. The pipeline then combines the raw data into a datacube that includes the variance of every pixel at every wavenlength. Consequently, we subtracted the remainder of the sky at every wavelength by measuring the median offset in 11 blank areas at every wavelength, and subtracting this from the entire field. We measured a spatial FWHM of $1.1^{\prime \prime}$ in the south-western datacube and a FWHM of $<1.0^{\prime \prime}$ in the north-eastern datacube on a point like source selected from HST images in both pointings.

For each pointing, we used a spectrally collapsed image of the datacube to find sources. In addition to this, we visually inspected the datacube to find sources with emission lines that were not visible in the stacked image. Further, we used the HST images to look for bright galaxies not included in our list, or galaxies that were only visible in either or both of the $F 606 \mathrm{~W}$ and $F 814 \mathrm{~W}$ bands, as this is often a good indication that the source is

\footnotetext{
9 ESO Programme 060.A-9345, PI Caputi \& Grillo.
}

10 ESO Programme 095.A-0653, PI Caputi. at high redshift. Finally, we used the predictions from our lensing models to search for additional images of lensed LAEs in MUSE observations. At each of these positions, we then extracted a spectrum with an aperture of $1^{\prime \prime}$ radius to determine the redshift of the galaxy.

\section{Spectral analysis}

We presented the redshifts obtained from the first south-western (SW) pointing in Paper I. Here we complement those measurements with the new redshifts determined for the north-eastern (NE) half of AS1063. In Appendix A we provide a complete compilation of all the redshifts obtained from our two MUSE pointings. We determined redshifts for three additional highredshift galaxies with multiple images, of which two were described in C16. The third system is a $z=3.606 \mathrm{LBG}$, with weak Ly $\alpha$ emission and two images within the south western MUSE pointing. This system, labelled as SW-70, has a clear continuum and several UV absorption features clearly visible in both images, see Fig. 4.

We selected all the LAEs that we found in the observations, see Table 1, resulting in 6 and 8 LAEs behind the south-western half and the north-eastern half of AS1063 respectively. Two of these LAEs were discussed in more detail in Vanzella et al. (2016a) and Caminha et al. (2016a). The first is an opticallythin, young, and low-mass galaxy that is a good candidate for a Lyman continuum emitter, which we studied using the expanded wavelength range and higher resolution spectroscopy of $\mathrm{X}$-SHOOTER. The second LAE is accompanied by an extended Ly $\alpha$ nebula, for which we found the most likely origin is scattered Ly $\alpha$ photons emitted by embedded star formation.

We determined the redshifts for 116 objects in the NE of AS1063, belonging to 102 individual galaxies. We found 6 foreground objects, 74 galaxies that belong to the cluster, and 22 galaxies behind the cluster. We identified 10 galaxies that show multiple images, for a total of 25 images, including the two images of the quintiply lensed $z=6.11 \mathrm{LAE}$ which still lacked spectroscopic confirmation. Combining these redshifts with the 
W. Karman et al.: Properties of low luminosity LAEs at $z>3$
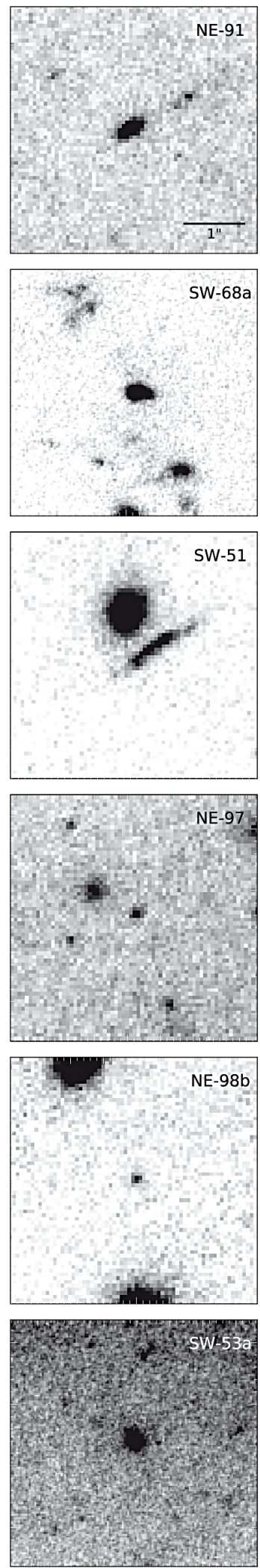
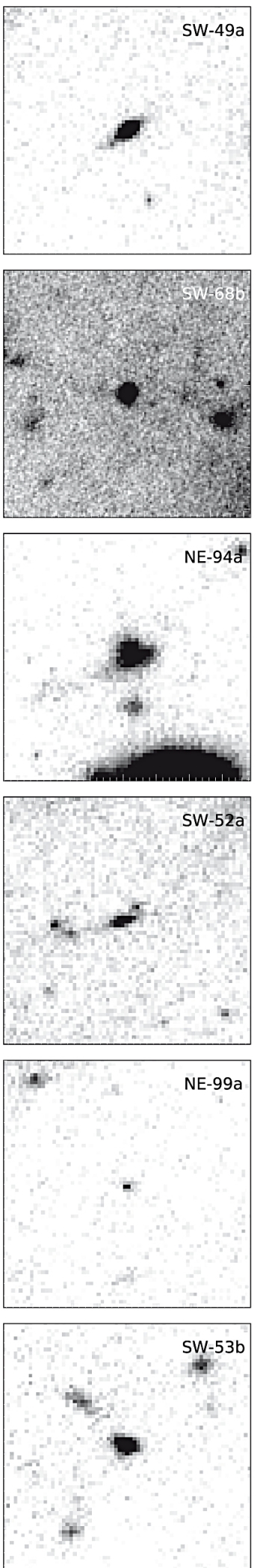
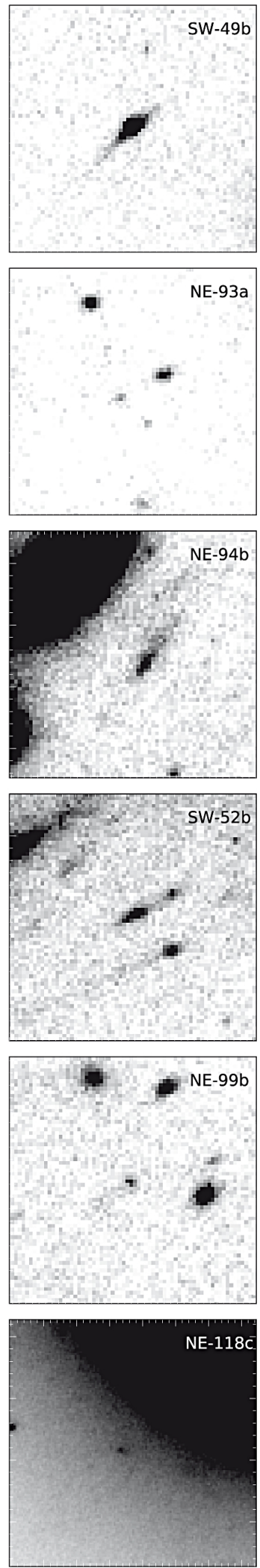
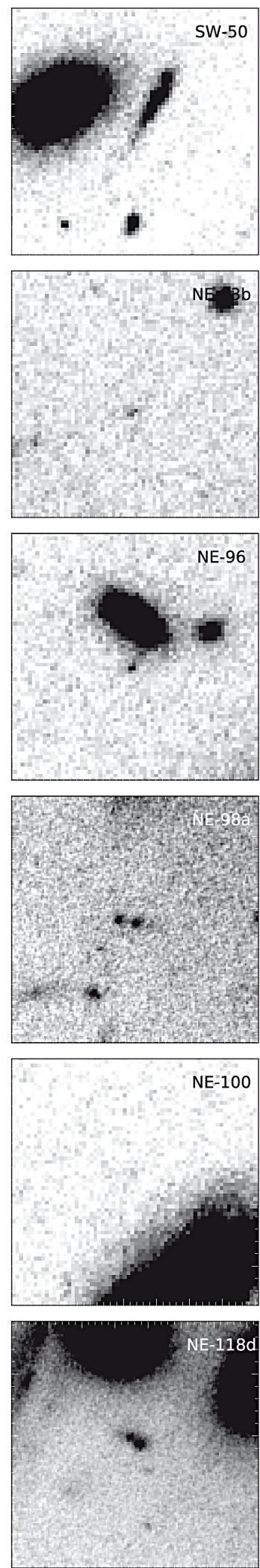

Fig. 2. HST $F 814 W$ stamps of all LAEs in the MUSE footprint. The ID of each LAE is plotted in the top right corner of each panel. Each stamp is $4^{\prime \prime}$ on each side, and a scalebar with a size of $1^{\prime \prime}$ is shown in the top left image.

SW MUSE observations, results in a total of 9 foreground objects, 121 cluster galaxies, and 42 background galaxies with MUSE redshifts in the central region of AS1063. We do not find any high-ionization UV emission lines for any of the new LAEs.
The total number of spectroscopically confirmed multiple image systems in AS1063 has been increased from 10 to 18, with 17 systems having at least 2 redshifts spectroscopically determined with MUSE, see Table B.1. We find one additional faint 
Table 1. LAEs behind AS1063, see Table A.1 for quality flags and a cross correlation with multiple images.

\begin{tabular}{cccc}
\hline \hline ID & RA (J2000) & Dec (J2000) & $z$ \\
\hline NE-91 & 342.19238 & -44.52505 & 2.9760 \\
SW-49a & 342.17505 & -44.54102 & $3.1169^{c, e}$ \\
SW-49b & 342.17315 & -44.53999 & $3.1169^{a, b, c, e, f}$ \\
SW-50 & 342.16225 & -44.53829 & 3.1160 \\
SW-68a & 342.18745 & -44.53869 & $3.1166^{a, h}$ \\
SW-68b & 342.17886 & -44.53587 & $3.1166^{a, h}$ \\
NE-93a & 342.18283 & -44.52028 & 3.1690 \\
NE-93b & 342.19196 & -44.52409 & 3.1690 \\
SW-51 & 342.17402 & -44.54124 & 3.2275 \\
NE-94a & 342.18935 & -44.51871 & 3.2857 \\
NE-94b & 342.19615 & -44.52291 & 3.2857 \\
NE-96 & 342.19709 & -44.52483 & 3.4514 \\
NE-97 & 342.19100 & -44.52679 & 3.7131 \\
SW-52a & 342.18150 & -44.53936 & $4.1130^{c}$ \\
SW-52b & 342.17918 & -44.53870 & $4.1130^{c}$ \\
NE-98a & 342.19015 & -44.53093 & 5.0510 \\
NE-98b & 342.19085 & -44.53566 & 5.0510 \\
NE-99a & 342.18378 & -44.52122 & 5.2373 \\
NE-99b & 342.18874 & -44.52276 & 5.2373 \\
NE-100 & 342.19701 & -44.52212 & 5.8940 \\
SW-53a & 342.18106 & -44.53462 & $6.1074^{b, c, g}$ \\
SW-53b & 342.19088 & -44.53747 & $6.1074^{b, c, g}$ \\
NE-118c ${ }^{\dagger}$ & 342.18402 & -44.53159 & 6.1074 \\
NE-118d ${ }^{\dagger}$ & 342.18904 & -44.53004 & 6.1074 \\
\hline SW-70a & 342.18586 & -44.53883 & 3.6065 \\
SW-70b & 342.17892 & -44.53668 & 3.6065 \\
\hline
\end{tabular}

Notes. The last galaxy, SW-70, is no LAE, but a Lyman Break galaxy with minimal Ly $\alpha$ emission, and is therefore not considered in the remainder of this paper. Previous redshift determinations by: ${ }^{(a)}$ C16; ${ }^{(b)}$ Balestra et al. (2013); ${ }^{(c)}$ Paper I; ${ }^{(d)}$ Richard et al. (2014); (e) Vanzella et al. (2016a); $(f)$ Johnson et al. (2014); ${ }^{(g)}$ Boone et al. (2013); and ${ }^{(h)}$ Caminha et al. (2016a). ${ }^{(\dagger)}$ NE-118 is the same image family as SW-53 but located in the NE rather than the SW. To avoid confusion with object NE-53, we listed the objects with the NE-118 identifier.

line emitter which we associate with Ly $\alpha$ emission at $z=5.894$, which has no clear counterpart in the FF images. The lensing model predicts additional images outside of the observed MUSE field, but their magnifications are too low to be detected in the HST images. The addition of 2 and possibly 3 spectroscopically confirmed systems at $z>5$ should help to further constrain the cosmological parameters, see $\mathrm{C} 16$, while the increased number of $z=3-4$ images will decrease the degeneracies and uncertainties in the models. We corrected all properties in the main body of this paper for gravitational lensing magnification, using the model described in Appendix B.

Due to lensing distortions, most of the galaxies discussed here have irregular morphologies in the image plane. To optimise the $\mathrm{S} / \mathrm{N}$ of the Ly $\alpha$ line, we created a spatial mask for each source, and extracted the spectrum within this mask. Each mask was constructed by collapsing the cube over the spectral width of the Ly $\alpha$ line, smoothing this stacked image by a 3 pixel wide boxcar function, and masking out every pixel with values $<5 \sigma$ off the background. We verified that this effectively masked out nearby contaminating sources, while also selecting the entire region of $\operatorname{Ly} \alpha$ emission.

In Fig. 3, we show the observed line profiles of all LAEs discovered in the datacubes, uncorrected for magnification. It is clear from this figure that the observed fluxes vary widely, from very bright (SW-49) to very faint (NE-97) Ly $\alpha$ lines. We see that all profiles have an asymmetry typical for Ly $\alpha$ lines, and most show a clear smaller blue peak or suggest the presence of a small blue peak. The width of the lines varies amongst our sample, but we find that most LAEs have narrow emission lines. Such narrow lines suggest the presence of low column density gas, and are suggested to be candidate Lyman continuum emitters (e.g. Jones et al. 2013; Verhamme et al. 2015; Vanzella et al. 2016a; Dijkstra et al. 2016).

\subsection{Physical properties deduced from Lyo}

The LAEs studied here belong to the intrinsically-faintest galaxies $\left(f_{\lambda}=36-2500 \times 10^{-20} \mathrm{erg} \mathrm{s}^{-1} \mathrm{~cm}^{-2}\right)$ spectroscopically confirmed at these redshifts, see Fig. 5. We measured the Ly $\alpha$ luminosity by summing the flux over the spectroscopic width of the $\operatorname{Ly} \alpha$ line, and subtracting the average of uncontaminated spectral regions redwards and bluewards of Ly $\alpha$. Subsequently, we used our lensing models to correct the luminosities for the magnifications due to the galaxy cluster, see Table C.1 for the adopted magnification factors. The errors on the magnifications are typically of the order of 5-10\%, which are generally larger than the photometric errors. For clarity, we have not propagated the errors on our magnification into our error estimates of other properties in any table or figure. An additional error based on the magnification is therefore applicable for flux derived properties, such as luminosities and stellar masses.

As we have multiple images for some galaxies, we can perform a test on our luminosities and magnifications. We compared the obtained luminosities for these objects, and used the mean luminosity when they agreed within $2 \sigma$. For those sources where a larger difference was found, we reinvestigated the datacube, and found that the lower-luminosity objects were underestimated. For two of these, the underestimation was due to the proximity of the edge, which resulted in only a partial coverage. For 3 other objects, contamination by nearby cluster members resulted in an oversubtraction of the continuum, while for 1 object a lower $\mathrm{S} / \mathrm{N}$ in combination with proximity to a skyline resulted in lower fluxes.

\subsubsection{Line profile modelling}

In addition to $L_{\mathrm{Ly} \alpha}$, we used our spectra to obtain physical properties of the gas surrounding these faint galaxies. We used the Ly $\alpha$ line fitting pipeline described in detail in Gronke et al. (2015) which consists of a pre-computed grid of Ly $\alpha$ radiative transfer models on an expanding shell and a Bayesian fitting framework.

The expanding shell model (first used by Ahn et al. 2003) consists of a central $\operatorname{Ly} \alpha$ (and continuum) emitting source surrounded by an outflowing shell of hydrogen and dust. Such a model has six free parameters: two describing the photon emitting source (the intrinsic line width $\sigma_{\mathrm{i}}$ and equivalent width $E W_{\mathrm{i}}$ ), three for the shell content (the neutral hydrogen column density $N_{\mathrm{HI}}$, the dust optical depth $\tau_{\mathrm{d}}$ and the effective temperature $T$ which includes the approximate effect of turbulence) and the outflow velocity $v_{\text {exp }}$.

The pre-computed grid mentioned above consists of 10800 models $^{11}$ covering the three parameters $T, N_{\mathrm{HI}}$ and $v_{\exp }$ as they shape the spectrum in a complex, non-linear fashion

\footnotetext{
${ }^{11}$ The spectra can be accessed online at http://bit.ly/ man-alpha/
} 
W. Karman et al.: Properties of low luminosity LAEs at $z>3$

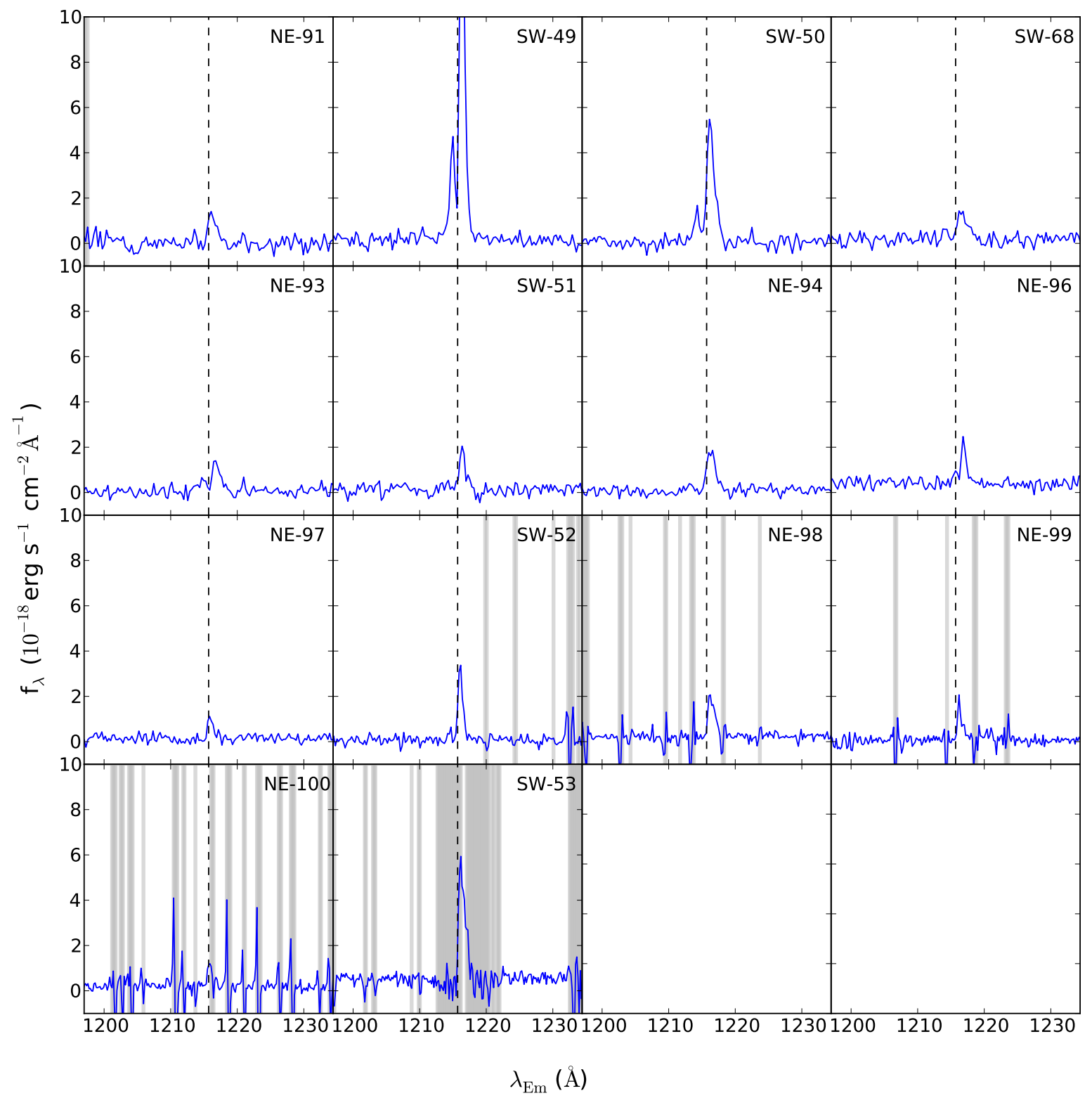

Fig. 3. Ly $\alpha$ lines for the LAEs extracted from the MUSE datacube, shown by the blue lines. The spectra are shifted to restframe wavelengths, and the fluxes are not corrected for the gravitational magnification. The grey bands show wavelengths with significant sky interference, while the black dashed line shows the restframe wavelength of Ly $\alpha$. The systemic redshifts of LAEs SW-49 and SW-68 have been determined from the narrow UV-emission lines, while we adopted the redshifts based on the Ly $\alpha$ line for the other objects.

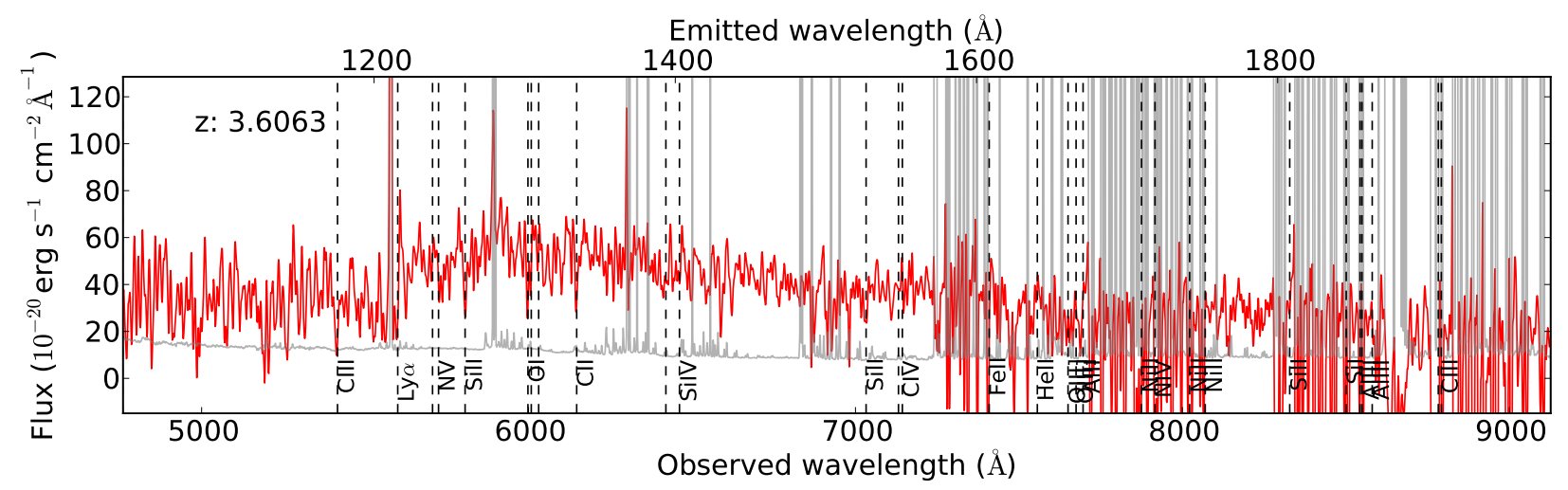

Fig. 4. Spectrum of object SW-70a, a newly identified LBG in the SW of AS1063. The spectrum has been smoothed for illustrative purposes, the grey bands correspond to wavelengths with significant sky interference. 


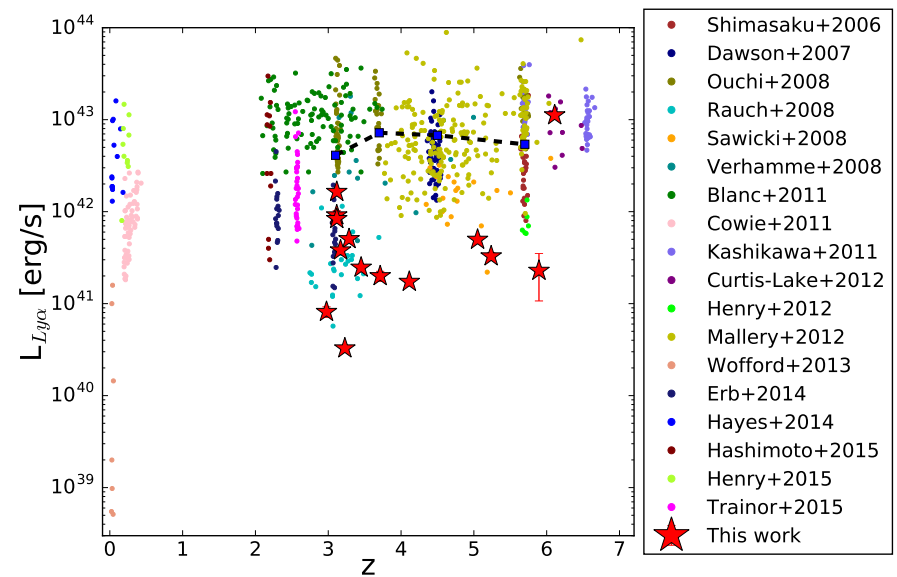

Fig. 5. Delensed luminosity of the Ly $\alpha$ line against the redshift of our targets, marked by the red stars. We compare this to previously large samples of spectroscopically-confirmed LAEs in the literature, which are shown by dots of different colours. We overplot the values of $L^{\star}$ at various redshifts from Ouchi et al. (2008) with a black dashed line.

(Verhamme et al. 2015). The grid was created using the radiative Monte Carlo code tlac (Gronke \& Dijkstra 2014) which traces individual photon packages in real- and frequency space (for a comprehensive review on $\operatorname{Ly} \alpha$ radiative transfer see, e.g., Dijkstra 2014).

The effect of the remaining three parameters is modelled in post-processing by assigning a weight to each individual photon packet, which means that the procedure affects the shape of the line and not only the normalization. This strategy does not only save computational time but allows to model these parameters continuously, and thus, leads to a more precise sampling of the likelihood when comparing the modelled data to observations.

The actual fitting procedure is done by sampling the Gaussian likelihood using the affine invariant Monte-Carlo sampler emcee (Foreman-Mackey et al. 2013) using 400 walkers and 600 steps $^{12}$. In addition to the minimal set of the six shell-model parameters, we also fit simultaneously for the redshift $z$ and the full-width at half maximum of the Gaussian smoothing kernel FWHM. Note that the former adds immense complexity to the fitting procedure as shifting $z$ by a small fraction can alter the quality of the fit tremendously. We used the redshift estimate from UV emission lines if available or otherwise the redshift of Ly $\alpha$ with an intrinsic uncertainty of $\sim 200 \mathrm{~km} \mathrm{~s}^{-1}$ (see Sect. 3.1) as a prior. Alternatively, the latter, i.e. smoothing the spectrum, makes the likelihood function better behaved. However, the width of the smoothing kernel is a function of the actual size of the Ly $\alpha$ halo as well as the measurement aperture. Therefore, we used an allowed range for FWHM corresponding to the wavelength-dependent spectral resolution of the MUSE instrument.

Gronke et al. (2015) discussed the uncertainties of using Ly $\alpha$ line profile fitting for various effects, for example morphology and signal-to-noise ratio. They showed that the expansion velocity and column density can be recovered reasonably well in most cases, while degeneracies and uncertainties are more prominent among the other parameters. Therefore, we focus in this paper on these two quantities, although we give the full fitting results in Appendix D. This is, to our knowledge, the first

\footnotetext{
${ }^{12}$ For particularly difficult, multi-modal cases we used a parallel tempered ensemble MCMC sampler (for a review see, Earl \& Deem 2005) with 20 temperatures, 50 walkers and 3000 steps.
}

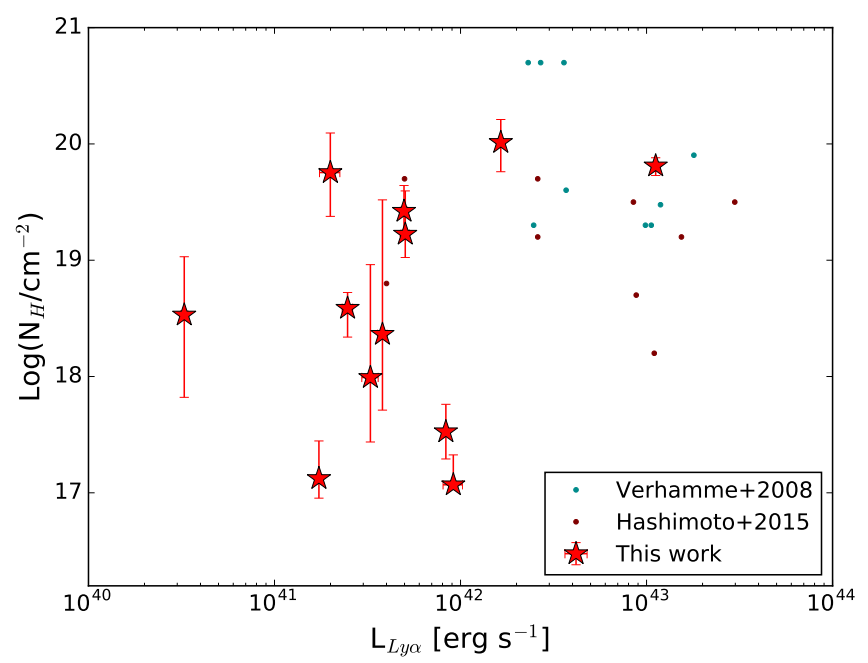

Fig. 6. Delensed luminosity in Ly $\alpha$ against the column density determined from modelling the $\operatorname{Ly} \alpha$ profile. Legend and symbols are as in Fig. 5.

time that the gaseous properties of such faint sources are studied through Ly $\alpha$ modelling. Therefore, this presents one of the first studies to determine the effect star formation has on gas in these faint galaxies.

\subsubsection{Shell properties}

We modelled the Ly $\alpha$ profiles of 12 LAEs using the approach described above yielding excellent fits to the observed Ly $\alpha$ spectra (see Appendix D). We present the properties based on the Ly $\alpha$ line in Table 2, where we already combined the multiple images into a single result. We modelled the Ly $\alpha$ line profile of each image, and combined the results of each modelling into a single result per LAE. We used the average of all images, after discarding multiple images which were possibly affected by close galaxies or artefacts in the datacube. We did not include 2 LAEs in the modelling, as their Ly $\alpha$ lines were too faint and spectrally unresolved.

In Fig. 6, we show that the column density of the expanding shell is low in all of the galaxies. In Vanzella et al. (2016a) we reported the results of Ly $\alpha$ line modelling for SW-49 using higher resolution X-shooter spectra and an updated shellmodel fitting pipeline. We find that the MUSE and X-shooter results are broadly consistent within the error bars, although we used a larger database reaching lower column densities for the $\mathrm{X}$-shooter spectrum.

We compare the best fit column densities in our sample to those found by Verhamme et al. (2008) and Hashimoto et al. (2015), who also fit Ly $\alpha$ profiles with expanding shell models. With all except one LAE best fit with $\log \left(N_{\mathrm{H}} / \mathrm{cm}^{2}\right)<20$ and only five LAEs with $\log \left(N_{\mathrm{H}} / \mathrm{cm}^{2}\right)>19$, we find lower column densities than the other studies. However, the galaxies studied here are also $\sim 1$ dex fainter than the ones presented in Verhamme et al. (2008) and Hashimoto et al. (2015).

These low column densities support the idea that for these faint galaxies, the Ly $\alpha$ is not significantly broadened by scattering. This is consistent with the picture that Ly $\alpha$ escape becomes easier for fainter galaxies with significant $\operatorname{Ly} \alpha$ emission.

We find no trends in the velocity of the expanding shell with the Ly $\alpha$ luminosity, but we note that we find relatively low outflow velocities in all galaxies. The absence of a correlation 
W. Karman et al.: Properties of low luminosity LAEs at $z>3$

Table 2. Properties derived from Ly $\alpha$ spectroscopy, after averaging results for multiple images.

\begin{tabular}{llllll}
\hline \hline ID & $z$ & $\begin{array}{l}f_{\text {Ly } \alpha}{ }^{a} \\
10^{-20} \mathrm{erg} / \mathrm{s}^{-\mathrm{cm}^{-2}}\end{array}$ & $\log \left(L_{\mathrm{Ly} \alpha} /(\mathrm{erg} / \mathrm{s})\right)^{b}$ & $\log \left(N_{\mathrm{H}} / \mathrm{cm}^{-2}\right)^{c}$ & $v_{\text {exp }}{ }^{d}$ \\
\hline NE-91 & 2.9760 & $108 \pm 14$ & $40.91 \pm 0.06$ & - & - \\
SW-49 & 3.1166 & $1080 \pm 129$ & $41.91 \pm 0.06$ & $17.07_{-0.03}^{+0.26}$ & $66.78_{-6.11}^{+7.07}$ \\
SW-50 & 3.1169 & $984 \pm 18$ & $41.92 \pm 0.01$ & $17.53_{-0.23}^{+0.24}$ & $123.63_{-5.85}^{+6.57}$ \\
SW-68 & 3.1166 & $1942 \pm 16$ & $42.22 \pm 0.01$ & $20.01_{-0.25}^{+0.20}$ & $463.18_{-48.58}^{+20.40}$ \\
NE-93 & 3.1690 & $431 \pm 26$ & $41.58 \pm 0.02$ & $18.36_{-0.65}^{+1.16}$ & $44.65_{-1.79}^{+27.91}$ \\
SW-51 & 3.2271 & $36 \pm 2$ & $41.24 \pm 0.01$ & $18.53_{-0.71}^{+0.50}$ & $97.99_{-17.76}^{+14.63}$ \\
NE-94 & 3.2857 & $524 \pm 27$ & $41.70 \pm 0.02$ & $19.22_{-0.20}^{+0.37}$ & $183.33_{-8.92}^{+23.37}$ \\
NE-96 & 3.4514 & $228 \pm 15$ & $41.39 \pm 0.03$ & $18.59_{-0.25}^{+0.14}$ & $56.68_{-8.85}^{+7.35}$ \\
NE-97 & 3.7131 & $155 \pm 19$ & $41.30 \pm 0.06$ & $20.39_{-0.14}^{+0.09}$ & $180.58_{-13.00}^{+13.00}$ \\
SW-52 & 4.1130 & $106 \pm 4$ & $41.24 \pm 0.02$ & $17.12_{-0.17}^{+0.32}$ & $98.10_{-10.29}^{+12.93}$ \\
NE-98 & 5.0510 & $186 \pm 8$ & $41.70 \pm 0.02$ & $19.42_{-0.21}^{+0.22}$ & $149.27_{-11.83}^{+11.83}$ \\
NE-99 & 5.2373 & $113 \pm 11$ & $41.47 \pm 0.05$ & $17.99_{-0.56}^{+0.97}$ & $107.87_{-127.68}^{+223.75}$ \\
NE-100 & 5.8940 & $60 \pm 32$ & $41.36 \pm 0.33$ & - & - \\
SW-53 & 6.1074 & $2694 \pm 67$ & $43.04 \pm 0.01$ & $19.81_{-0.08}^{+0.07}$ & $150.56_{-4.1}^{+3.69}$ \\
\hline
\end{tabular}

Notes. The columns are ${ }^{(a)}$ lens-corrected Ly $\alpha$ flux; ${ }^{(b)}$ luminosity; ${ }^{(c)}$ the hydrogen column density, and ${ }^{(d)}$ expansion velocity.

Table 3. Parameter space used for constructing stellar templates used in our SED fitting.

\begin{tabular}{lll}
\hline & Range & Nr. steps \\
\hline Age & $0.01 \mathrm{Myr}-2.3 \mathrm{Gyr}$ & 50 \\
$E(B-V)$ & $0-1.5$ & 20 \\
$Z$ & $0.0004-0.02\left(Z_{\odot}\right)$ & 4 \\
$\tau$ & $0.001 \mathrm{Gyr}-5 \mathrm{Gyr}$ & 5 \\
\hline
\end{tabular}

Notes. The stepsizes are logarithmic distributed for the ages, while we use an irregular spacing for the stepsizes of $E(B-V)$ where we finely sample the low values, and use larger steps for the higher values. The metallicities correspond to the $m 32, m 42, m 52$ and $m 62$ models of BC03.

between the Ly $\alpha$ luminosity and the expansion velocity of the shell is unexpected, as both are considered to correlate with the SFR (e.g. Weiner et al. 2009; Bradshaw et al. 2013; Chisholm et al. 2015). A possible explanation could be that the outflow speed does not follow the SFR at low masses, see below, or that the Ly $\alpha$ luminosity depends more strongly on the escape fraction of Ly $\alpha$ photons than on the SFR.

\section{Stellar properties}

We used the constructed photometric catalogue in combination with the spectroscopic redshifts to perform a spectral energy distribution (SED) fitting on our selected sample. We used LePhare (Arnouts et al. 1999; Ilbert et al. 2006) in combination with Bruzual \& Charlot (2003, hereafter BC03) templates to fit the photometry with stellar population models (see Table 3 for our model parameters). The set of stellar populations consists of an exponentially declining star formation histories, $S F R(t)=$ $S F R_{0} \times \mathrm{e}^{-t / \tau}$, with different values for $\tau$. In addition, we created templates with three different metallicities, and ages up to the age of the Universe. We used a Calzetti et al. (2000) extinction curve to attenuate all stellar templates, with $0<E(B-V)<$ 1.5. We enabled adding nebular emission lines to the templates based on their UV luminosity as described in Ilbert et al. (2009), where the line fluxes of [O II] $\lambda 3727$ are derived using the current SFR, and [O III] $\lambda \lambda 4959,5007, \mathrm{H} \alpha$, and $\mathrm{H} \beta$ are then scaled from locally derived line ratios. The addition of emission lines to stellar-population templates is shown to be important in the high-redshift Universe (e.g. Schaerer \& de Barros 2009; de Barros et al. 2014).

We fitted the SED of each image and combined the results of the multiple images in a similar method as for the Ly $\alpha$ luminosities and $\operatorname{Ly} \alpha$ line fitting. We used the average results of multiple images when the quality of the photometric data of each image is similar, but adopt the results of only the best constrained image if the difference is significant, for example for NE-94 we only used image a. For galaxies where we suspect contamination from nearby galaxies, for example SW-68b, we used only the images without contamination. We performed tests on the reliability of our results in Appendix C, and found that although there are few constraints in the restframe optical, our results do not change significantly.

We present the results from the SED fitting in Table 4. We remind the reader that most of the photometry is in the restframe UV, but that the Hawk-I and IRAC filters trace the restframe optical. We detected 6 LAEs in the $K$-band and $1 \mathrm{LAE}$ in the IRAC filters, but the non-detections provide important upperlimits when determining stellar masses and show that there is no hidden dominant old population of stars.

The masses we derived from our SED fitting are very low, varying from $\sim 10^{6} M_{\odot}$ to $\sim 10^{8} M_{\odot}$, significantly lower than the stellar masses explored in previous studies of LAEs. This is not surprising, as these galaxies are among the intrinsically faintest discovered so far with absolute UV-magnitudes ranging form 19 to -14 , and illustrates once more the advantages of gravitational lensing. We note that for 2 LAEs discovered here (NE-99 
Table 4. Stellar properties derived from SED modelling using LEPHARE in combination with BC03 templates.

\begin{tabular}{|c|c|c|c|c|c|c|c|}
\hline ID & $\log \left(M_{\star} / M_{\odot}\right)$ & $\log \left(\mathrm{Age}_{\mathrm{SSP}} / \mathrm{yr}\right)$ & $E(B-V)$ & $\log \left(S F R_{\mathrm{SED}} /\left(M_{\odot} \mathrm{yr}^{-1}\right)\right)$ & $\log (\mathrm{s} S F R / \mathrm{yr})$ & $\beta$ & $M_{\mathrm{UV}}$ \\
\hline NE-91 & $8.02_{-0.19}^{+0.20}$ & $8.26_{-0.67}^{+0.45}$ & $0.03_{-0.02}^{+0.03}$ & $-0.38_{-1.32}^{+0.16}$ & $-8.56_{-1.10}^{+0.39}$ & $-2.42 \pm 0.09$ & -17.77 \\
\hline SW-49 & $6.91_{-0.08}^{+0.18}$ & $6.70_{-0.06}^{+0.89}$ & $0.045_{-0.015}^{+0.03}$ & $-0.16_{-0.30}^{+0.90}$ & $-7.00_{-0.30}^{+0.80}$ & $-2.79 \pm 0.08$ & -17.09 \\
\hline SW-50 & $8.23_{-0.06}^{+0.05}$ & $6.28_{-0.63}^{+1.28}$ & $0.30_{-0.20}^{+0.00}$ & $1.88_{-1.99}^{+0.69}$ & $-6.34_{-2.82}^{+0.69}$ & $-1.95 \pm 0.05$ & -18.05 \\
\hline SW-68 & $7.78_{-0.16}^{+0.26}$ & $7.12_{-0.48}^{+0.69}$ & $0.10_{-0.05}^{+0.10}$ & $0.36_{-0.51}^{+0.67}$ & $-7.39_{-0.90}^{+0.66}$ & $-2.09 \pm 0.08$ & -18.18 \\
\hline NE-93 & $6.24_{-0.03}^{+0.27}$ & $6.49_{-0.03}^{+0.84}$ & $0.035_{-0.005}^{+0.036}$ & $-0.47_{-0.07}^{+0.91}$ & $-6.61_{-0.03}^{+0.80}$ & $-2.85 \pm 0.46$ & -15.63 \\
\hline SW-51 & $6.81_{-0.34}^{+0.49}$ & $7.59_{-0.65}^{+0.82}$ & $0.05_{-0.03}^{+0.05}$ & $-0.96_{-0.27}^{+0.30}$ & $-7.87_{-0.69}^{+0.63}$ & $-2.31 \pm 0.05$ & -15.83 \\
\hline NE-94 & $8.50_{-0.16}^{+0.16}$ & $6.70_{-0.66}^{+0.66}$ & $0.30_{-0.05}^{+0.05}$ & $1.61_{-0.59}^{+0.93}$ & $-6.74_{-0.75}^{+0.64}$ & $-1.77 \pm 0.08$ & -18.30 \\
\hline NE-96 & $7.33_{-0.44}^{+0.41}$ & $7.24_{-0.96}^{+0.87}$ & $0.10_{-0.07}^{+0.15}$ & $-0.36_{-0.64}^{+1.34}$ & $-7.46_{-1.26}^{+1.12}$ & $-2.14 \pm 0.22$ & -16.60 \\
\hline NE-97 & $6.35_{-0.17}^{+0.18}$ & $6.40_{-0.75}^{+0.72}$ & $0.03_{-0.02}^{+0.03}$ & $-0.18_{-0.69}^{+0.81}$ & $-6.47_{-0.85}^{+0.71}$ & $-2.90 \pm 0.15$ & -15.98 \\
\hline SW-52 & $6.36_{-0.25}^{+0.38}$ & $7.12_{-0.66}^{+0.53}$ & $0.03_{-0.02}^{+0.03}$ & $-0.90_{-0.33}^{+0.61}$ & $-7.26_{-0.88}^{+0.74}$ & $-2.50 \pm 0.28$ & -15.33 \\
\hline NE-98 & $6.46_{-0.09}^{+0.10}$ & $6.16_{-0.66}^{+0.36}$ & $0.01_{-0.01}^{+0.02}$ & $0.28_{-0.54}^{+0.66}$ & $-6.19_{-0.45}^{+0.68}$ & $-3.17 \pm 0.02^{a}$ & -16.19 \\
\hline NE-99 & - & - & - & - & - & - & -14.42 \\
\hline NE-100 & - & - & - & - & - & - & $>-14.70$ \\
\hline SW-53 & $7.47_{-0.00}^{+0.00}$ & $6.04_{-0.54}^{+0.30}$ & $0.00_{-0.00}^{+0.00}$ & $1.37_{-0.30}^{+0.60}$ & $-6.10_{-0.30}^{+0.60}$ & $-3.17 \pm 0.02^{a}$ & -18.97 \\
\hline
\end{tabular}

Notes. The properties were derived after averaging the results of multiple images of the same source, if applicable. See Appendix C for results of all individual LAEs. ${ }^{(a)}$ This is the maximal UV slope in our used templates, the photometric UV-slope is steeper and the small errors are therefore not representative but a result of our method of calculating $\beta$.

and NE-100), we recovered only a single detection through all deep FF filters, which is in the filter containing Ly $\alpha$. The completion of the NIR-imaging of AS1063 in the summer of 2016 should add more detections to these objects, and will better constrain the properties of these possibly even less massive galaxies.

In Fig. 7, we compare the stellar masses of our galaxies to their Ly $\alpha$ luminosities. We see that the lower masses are paired with lower luminosities. The low luminosities and masses found here in comparison to previous literature results confirm again that these objects probe a new region of parameter space.

The presence of such narrow and strong Ly $\alpha$ emission is already a clear indication that there is little dust present. We find that the median marginalised $E(B-V)$ values are all very small, with only 3 galaxies having $E(B-V)>0.1$, in agreement with previous studies (e.g. Atek et al. 2014).

The ages of these very low mass objects are relatively low, that is $1-100 \mathrm{Myr}$, see Fig. 8 . We find that only two galaxies have an age $>100 \mathrm{Myr}$, and age seems to decrease with redshift. The young stellar ages indicate that these are systems that are rapidly building up their mass. This is confirmed by the SFR that we obtain given the low stellar masses, see Fig. 9, as with the current SFR, most galaxies will double their mass within $10^{7}$ yrs. As a consequence of these young ages, the models produced by different values of $\tau$ are very similar. Therefore, the current data are unable to distinguish between the different star formation histories.

We compare the SED-derived SFR to the SFR extrapolated from the SFR-stellar mass relation determined for more massive galaxies, both at lower and similar redshift. We find that most of the LAEs fall above this relation if an extrapolation of the power-law relation described by Whitaker et al. (2014) is considered. Because very little is known about the SFR in

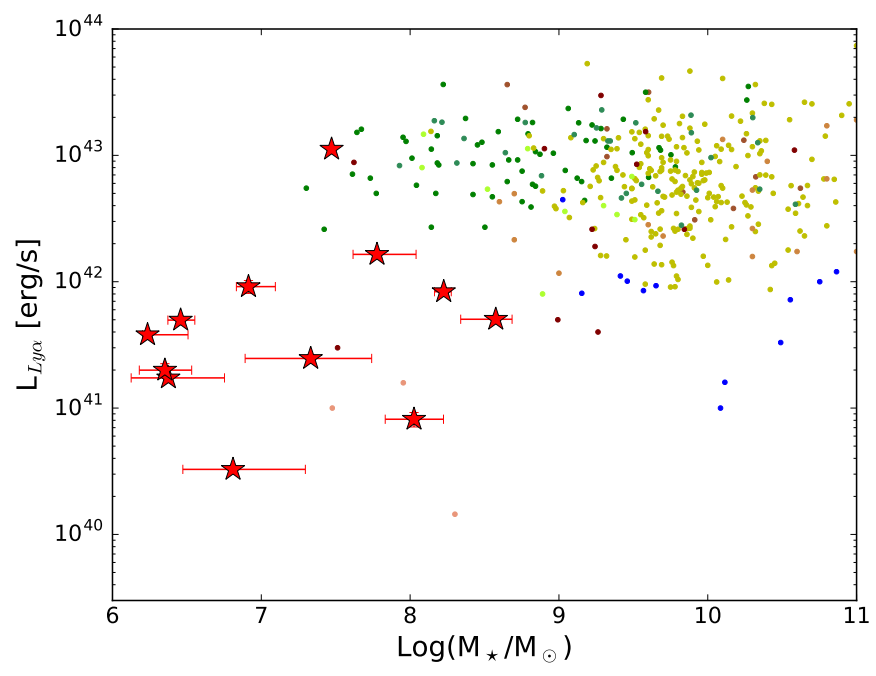

Fig. 7. Stellar mass versus the lens-corrected Ly $\alpha$ luminosity. The LAEs described here are compared to a collection of previous LAE studies, with the colours identical to Fig. 5, where we have supplemented the results from Blanc et al. (2011) with those of Hagen et al. (2014).

low mass galaxies, this extrapolation is rather uncertain due to a degeneracy between the slope of the power-law and its zeropoint. If we use the steep power-law relation described for $z \sim 4$ galaxies by Salmon et al. (2015), the number of LAEs above this relation will decrease by a factor 2 . We note however, that many studies favour a slope of $\alpha=1$ (e.g. González et al. 2010; Whitaker et al. 2014; Ilbert et al. 2015), which would make most of these LAE starbursting galaxies. 


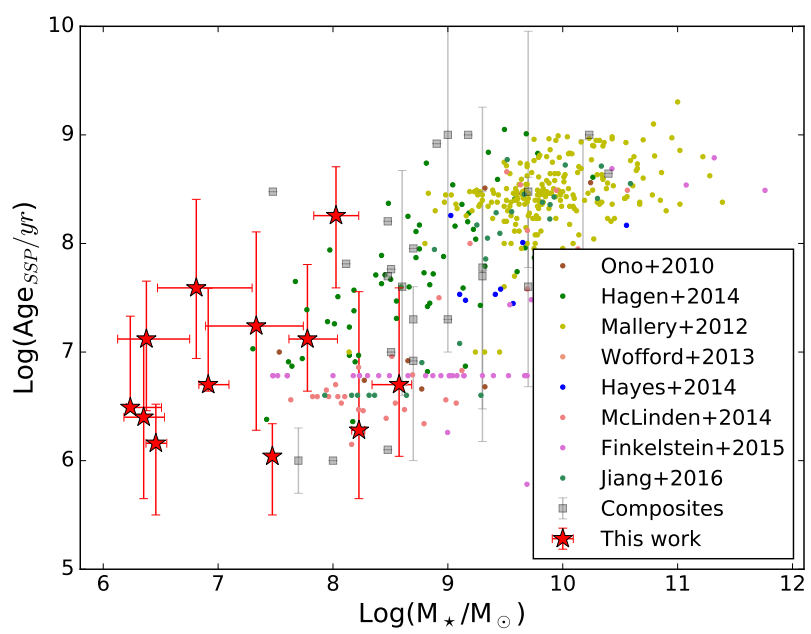

Fig. 8. Stellar mass versus the age of the stellar population, as determined by SED-fitting of exponentionally declining star formation histories. The coloured dots correspond to a variety of results from previous spectroscopically-confirmed LAE studies, while the grey squares correspond to the collection of composites assembled by McLinden et al. (2014). For comparison, we also plot a sample of non-LAE, represented by the pink dots (Finkelstein et al. 2015).

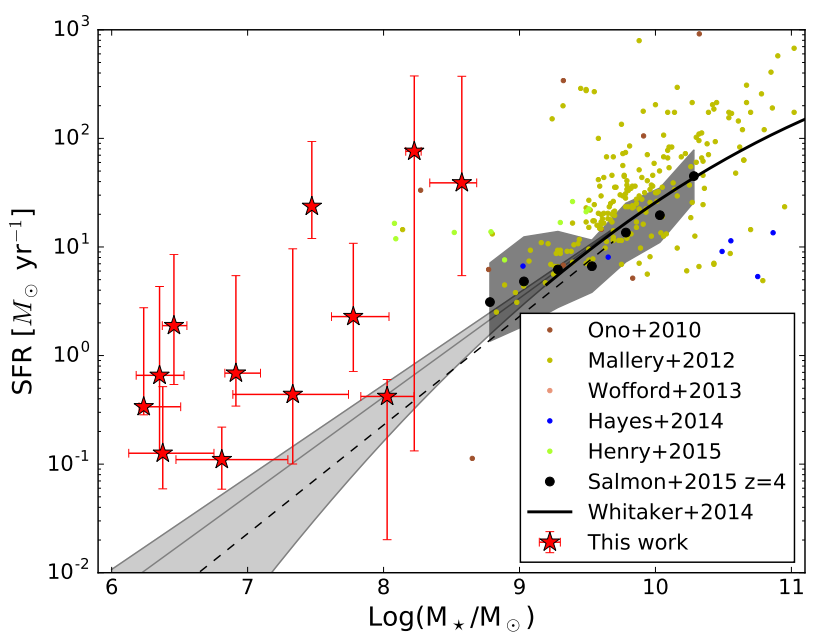

Fig. 9. Stellar mass versus the star formation rate. See Fig. 10 for a description of the different studies. The black dots and dark grey region correspond to the SFR for galaxies in CANDELS at $z \sim 4$ as determined by Salmon et al. (2015), while the black dashed line presents an extrapolation to lower masses based on these data. We show the SFR for a sample of $z=2.0-2.5$ galaxies from Whitaker et al. (2014) and three possible extrapolations to lower masses with the black line and three grey lines respectively.

To have a clearer understanding of whether these faint LAEs are normally star forming or starbursting, we looked at the specific SFR $\left(\mathrm{s} S F R=S F R / M_{\star}\right)$. We find that in our sample of LAEs the average sSFR is significantly higher than seen in other LAE samples, see Figs. 10 and 11. This shows that these galaxies are not only young, but are still actively forming stars. The sSFR classifies these galaxies as starburst galaxies, if one assumes the flat $\mathrm{SSFR}-M_{\star}$ correlation found for stellar masses $M_{\star}<10^{9}$ (González et al. 2010; Whitaker et al. 2014; Ilbert et al. 2015). However, whether this relation is flat at these redshifts is unknown, as this mass range has not been explored before even at low redshift.

Similarly high sSFRs are rarely found in any other sources. For more massive sources at high-redshift with young ages,

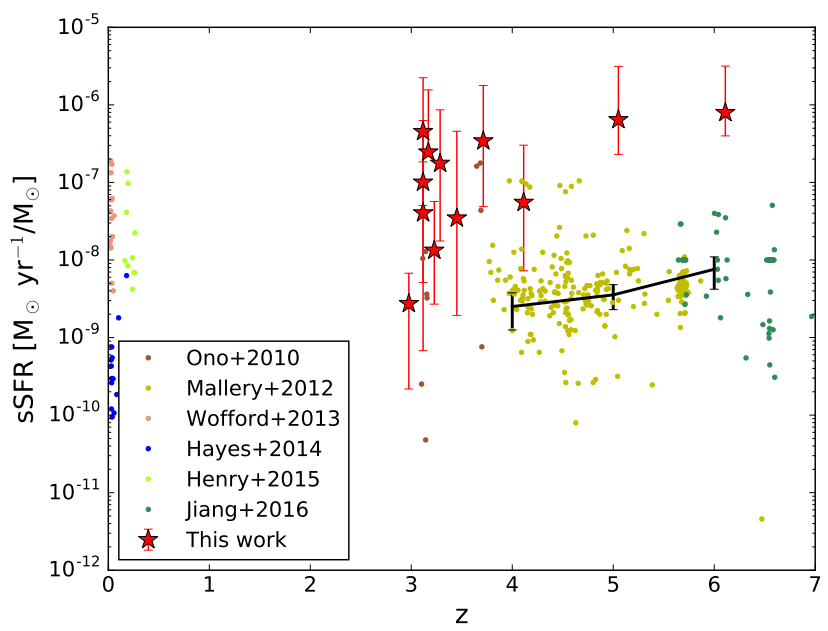

Fig. 10. Specific SFR as a function of redshift. The sSFR of our sample, Ono et al. (2010), Mallery et al. (2012), and Wofford et al. (2013) is calculated using only SED fitting, for Hayes et al. (2014), Henry et al. (2015) the sSFR is calculated using the SFR based on $\mathrm{H} \alpha$, and the sSFR of Jiang et al. (2016) is calculated using SFR $\mathrm{Uv}_{\mathrm{UV}}$. The black line shows the sSFR of the CANDELS galaxies from Salmon et al. (2015).

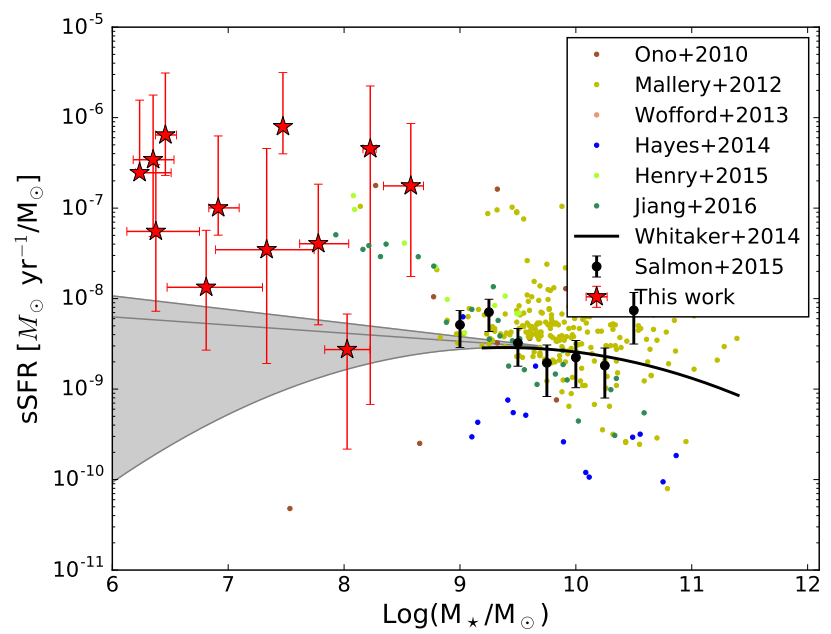

Fig. 11. Stellar mass versus the specific star formation rate. See Figs. 10 and 9 for a description of the different studies and legend.

a typical $\log (\mathrm{s} S F R)=-8$ is found (e.g González et al. 2010; Stark et al. 2013; Oesch et al. 2015, 2016; Tasca et al. 2015), although Ono et al. (2012) and Finkelstein et al. (2013) report a similarly high sSFR at $z>7$. Extreme emission line galaxies (EELGs) at lower redshift show similar values on average, but the spread is large enough that the upper envelope contains some galaxies with $\log (\mathrm{s} S F R)>-7.5$ (Cardamone et al. 2009; Amorín et al. 2014, 2015; Maseda et al. 2014; Ly et al. 2014). A comparable evolution of the sSFR with redshift as seen in normal galaxies (see Speagle et al. 2014, for a recent compilation), could bring the EELGs up to a similar sSFR found in our sample, strengthening the possible link between EELGs and highredshift LAEs.

We compared the sSFR to all other parameters, but find no further correlations with neither any gaseous nor any stellar property. Even the apparent trend of an increasing SSFR with increasing $L_{\mathrm{Ly} \alpha}$ within our sample disappears when other studies are added, see Fig. 12. This suggests that the sSFR has no influence on the $\operatorname{Ly} \alpha$ line profile or the physics that shape the line, 


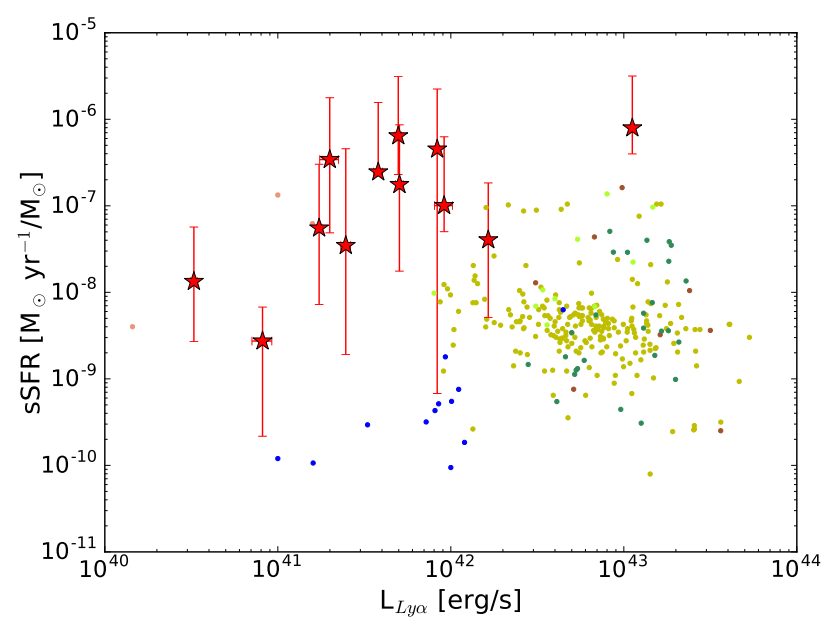

Fig. 12. Ly $\alpha$ luminosity versus the specific star formation rate. See Fig. 10 for a description of the different studies.

nor is the sSFR influenced by any other stellar parameter than the stellar mass and age. It is noteworthy that in several of these plots we find higher sSFR within our sample when compared to other samples at a given property. This further suggests that the high sSFR is driven by the low stellar mass and young age.

Most galaxies, except two (SW-50 and SW-51), are best fit with a low metallicity, however a solar metallicity falls within our $1 \sigma$ certainty range for most of the LAEs. A low metallicity for most of these galaxies would be in agreement with all previous results that these are recently formed, low-mass galaxies which are rapidly building up mass and have not been able to produce a large amount of metals.

\subsection{Ly $\alpha$ escape fraction}

To understand the evolution and formation of the Ly $\alpha$ luminosity function and the process of reionization it is important to determine how much of the Ly $\alpha$ flux escapes the galaxy. To estimate this, we follow previous studies, using the $\mathrm{UV}$ or SED derived $\mathrm{SFR}_{\mathrm{UV} / \mathrm{SED}}$ and compare these to the SFR calculated from $\operatorname{Ly} \alpha\left(\mathrm{SFR}_{\mathrm{Ly} \alpha}\right)$ to define the escape fraction $f_{\mathrm{esc}}=S F R_{\mathrm{Ly} \alpha} / S F R_{\mathrm{SED}}$. We determine $\mathrm{SFR}_{\mathrm{Ly} \alpha}$ following Kennicutt (1998) and assuming case B recombination:

$S F R_{\mathrm{Ly} \alpha}\left(M_{\odot} \mathrm{yr}^{-1}\right)=L_{\mathrm{Ly} \alpha}\left(\mathrm{erg} \mathrm{s}^{-1}\right) / 8.7 \times 7.9 \times 10^{-42}$.

We caution the reader that this relation has been calibrated on stable star forming galaxies and that this may not be applicable at very young ages.

In Fig. 13 we show the $\operatorname{Ly} \alpha$ escape fraction as a function of stellar mass. We find higher escape fraction for lower stellar masses, a trend which remains visible after other studies are included. We note, however, that this trend is also a natural effect of the selection bias when studying LAEs. Galaxies with low escape fractions will have very little or no Ly $\alpha$ emission, and will therefore remain undetected in emission line studies, whereas low mass galaxies will evade detection in the continuum. These effects would produce a trend similar to the one observed here, however, the dearth of massive galaxies with high-escape fractions is genuine and cannot be explained by selection effects.

\subsection{UV-continuum slope}

We used the best-fitting SED model to measure $\beta$, the slope of the UV spectrum defined as $f_{\lambda} \propto \lambda^{\beta}$ (e.g. Meurer et al. 1999),

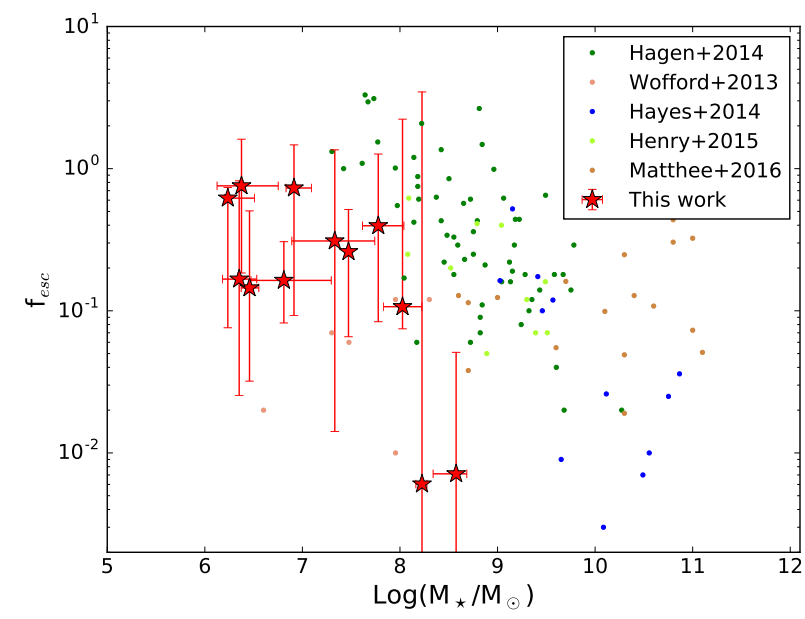

Fig. 13. Ly $\alpha$ escape fraction versus the stellar mass. We followed most studies and used the indirect $f_{\text {esc }}=S F R_{\mathrm{Ly} \alpha} / S F R_{\mathrm{SED}}$, while Hayes et al. (2014) and Henry et al. (2015) used the flux ratio of Ly $\alpha$ to $\mathrm{H} \alpha$ to directly derive $f_{\text {esc }}$.

following Finkelstein et al. (2012). We chose to use the bestfitting SED model to measure $\beta$ rather than observed colours as this was shown to be a more stable approach (e.g. Finkelstein et al. 2012; Bouwens et al. 2014). We adopted the spectral windows defined by Calzetti et al. (1994), and directly fitted a power law to the best-fitting template in these windows. We find a clear relation between the stellar mass and $\beta$, see Fig. 14, in agreement with previous results (e.g. Bouwens et al. 2014). For each galaxy, we derive the uncertainty of $\beta$ by repeating the full-SED modelling for 1000 mock galaxies, created by randomly disturbing its photometry taken from a normal random distribution based on the photometric errors. Because the minimum value of $\beta \approx-3.2$ for BC03 models with a Chabrier IMF, galaxies with photometric slopes bluer than this will almost always be best fitted with $\beta=-3.2$, even after scattering the photometry. This leads to unrealistically small errors for some of our UV-slopes, for example NE-98. We then fitted a Gaussian to the distribution of $\beta$ of all mock galaxies, and report the resulting $\sigma$ as the uncertainty in $\beta$. We calculated the slope of the $\beta$-mass relation to be 0.43 in our sample, comparable to the maximum slope of 0.46 at $z=7$ by Finkelstein et al. (2012). We note however, that the majority of our samples lies at $3<z<4$, for which Finkelstein et al. (2012) found a slope of only 0.17. An important factor in this difference could be our selection bias, meaning that we only find galaxies with strong Ly $\alpha$ emission.

Dunlop et al. (2012) argued that very blue UV slopes could be caused by low $\mathrm{S} / \mathrm{N}$ photometry. In this study however, we do not suffer from uncertainties in redshift, which are the largest factor in the scatter of $\beta$ in photometric studies. Therefore, we do not expect to be affected by the same bias. The very blue slopes are also in agreement with the low stellar masses, low dust extinction, and young ages found in these LAEs, as also discussed for example by Dunlop et al. (2012).

Jiang et al. (2016) measured the UV-slope of their sample of LAEs, and they find a significant number of more massive LAEs with very blue slopes. This seems to be in disagreement with our and other previous results, as they would predict that more massive galaxies have redder continua due to a higher metallicity and larger amounts of dust. This is possibly due to low $\mathrm{S} / \mathrm{N}$ photometric data available for the relevant sources in Jiang et al. (2016), leading to a larger scatter found in the $\beta$ slope (Dunlop et al. 2012). Another possible explanation for this is 


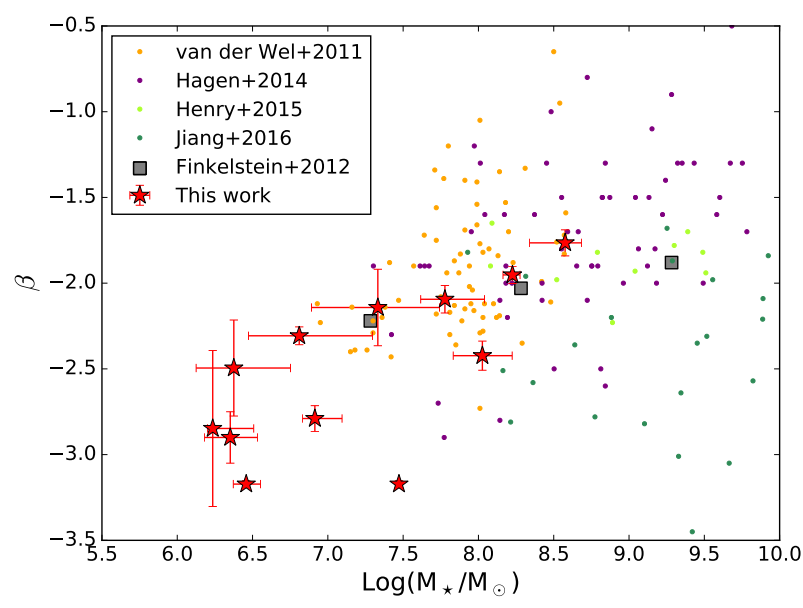

Fig. 14. Stellar mass versus the UV-continuum slope $\beta$. We overplotted a number of LAEs from Hagen et al. (2014), Henry et al. (2015), and Jiang et al. (2016), the median of CANDELS galaxies at $z=4$ from Finkelstein et al. (2012), and a sample of EELGs from van der Wel et al. (2011).

that the number density of blue more-massive LAEs is significantly lower than those of slightly redder LAEs. Because we are only observing a rather small volume, this means that the very low number of blue massive LAEs in our sample is expected.

We note that for a few of our LAEs, we find the steepest theoretically-allowed $\beta$ for pure stellar populations without a nebular contribution, that is $\beta=-3.17$. In these cases, the observations are representative of an even steeper slope, and, naturally, models with flatter continuum slopes are disfavoured by SED fitting. A steeper UV continuum than the theoretical maximum value for stellar templates $\beta<-3.2$ can be achieved by including the effects of a nebular continuum into the SED fitting (e.g. Schaerer \& de Barros 2009; Zackrisson et al. 2013) or by using top-heavy IMFs or Pop III stars (e.g. Bouwens et al. 2010; Zackrisson et al. 2011).

A steep UV-continuum slope is associated with young and rapidly star forming galaxies without a significant amount of dust. Therefore, one would expect that $\beta$ might correlate with the SFR. We show the comparison between SFR and $\beta$ in Fig. 15, but we see that there is no obvious correlation between the two either in our sample, or in either of the other samples. The lack of a clear relation between SFR and $\beta$ in our sample could possibly be due to the large uncertainty in the SFR derived from SED modelling, however, it has also been found in other studies. A physical reason for the absence of a correlation could be that the metallicity, and therefore the dust opacity, is not as strongly correlated with SFR as with stellar mass.

\subsection{Linking gas to stars}

We now compare the stellar properties derived from the broadband photometry to the gaseous properties derived from the Ly $\alpha$ line profile modelling. First, we compare the expansion velocity to the sSFR in Fig. 16. We find no clear relation between these two quantities, see Sect. 5 for a discussion on this.

A more interesting result is found when we compare the column density to the stellar mass, see Fig. 17. On the one hand, we see that the column density found by Hashimoto et al. (2015) using shell model fitting to $z \sim 2.2$ LAEs is similar to those found here, but at significantly higher stellar mass. On the other hand, the stellar masses of the $z \sim 0.03$ LAEs in Wofford et al. (2013), who use UV absorption line modelling for LBGs, are similar to

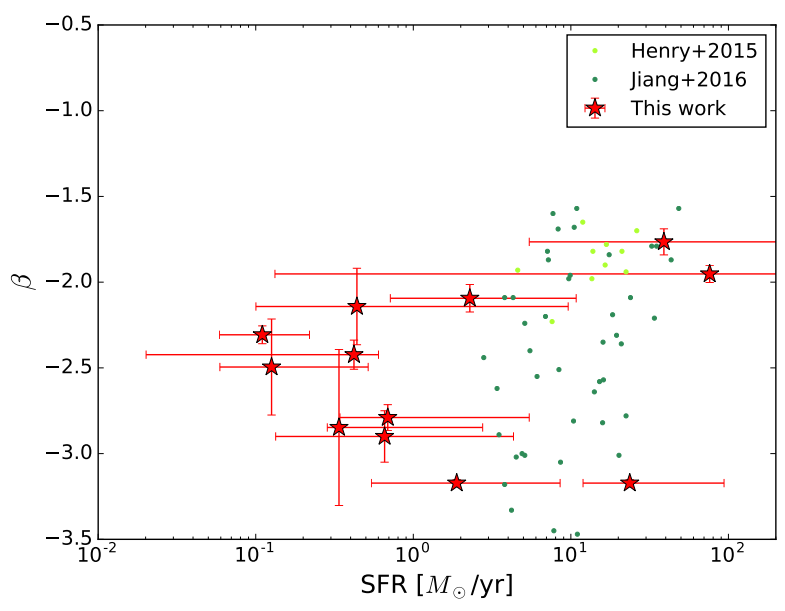

Fig. 15. SFR versus the UV-continuum slope $\beta$. We overplotted a number of LAEs from Henry et al. (2015) and Jiang et al. (2016).

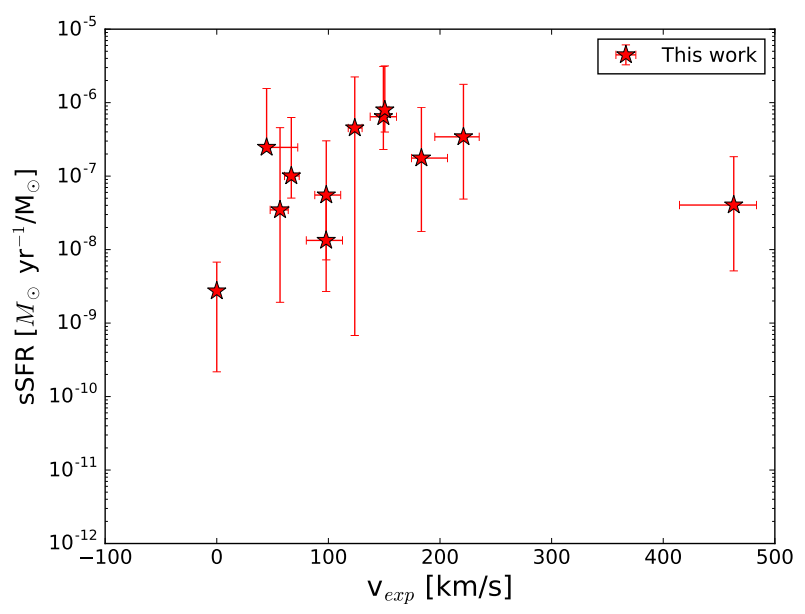

Fig. 16. Expansion velocity, derived from $\operatorname{Ly} \alpha$ line modelling, against the sSFR, derived from SED fitting.

the ones discussed here, but the column densities are two orders of magnitude larger. The difference could be a consequence of the different redshifts, as low column density objects are at relatively high-redshift when cosmic star formation was peaking, while the high column density refers to galaxies at $z<0.06$. This suggests an evolution of the column density of galaxies, although there are several caveats to be considered, see Sect. 5. We note, however, that none of the galaxies with column density measures from Wofford et al. (2013) are LAEs, opposed to the sample of Hashimoto et al. (2015) and the one discussed here. The difference in column densities at the same mass could therefore simply mean that selecting by Ly $\alpha$ luminosity sets a maximum value on the column density, which is in agreement with the finding of Hashimoto et al. (2015) that the expansion velocity and column density in LAEs are significantly lower than those in LBGs. It was shown by Schaerer et al. (2011) that a high expansion velocity $v_{\exp }>300 \mathrm{~km} \mathrm{~s}^{-1}$ is required in galaxies with a high column density in order to allow the escape of Ly $\alpha$ photons. These high velocities are significantly higher than expected for $M_{\star}<10^{8} M_{\odot}$ galaxies.

\section{Discussion}

The absence of a relation between the outflow velocity and the SFR, stellar mass, and sSFR is apparently in contradiction with previous studies (e.g. Weiner et al. 2009; Bradshaw et al. 2013; Erb et al. 2014). The difference in stellar masses could be the 


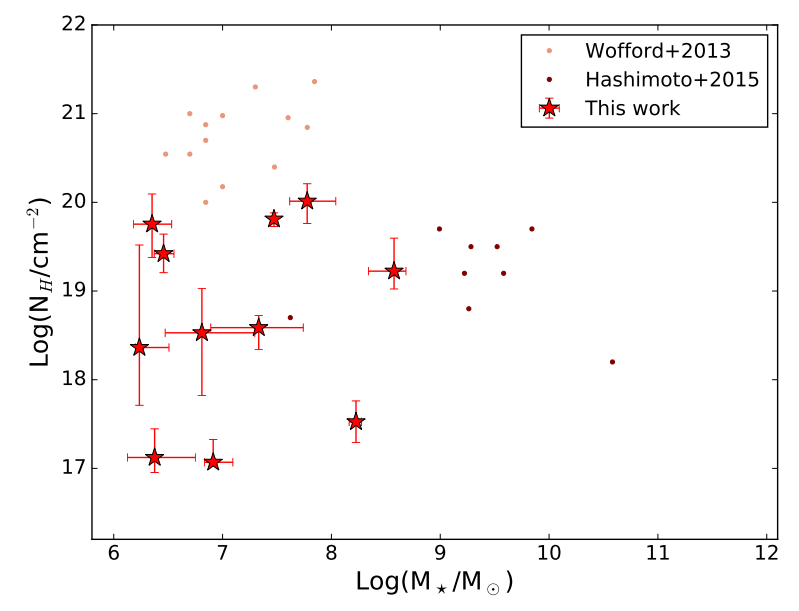

Fig. 17. Comparison of the stellar mass to the column density based on Ly $\alpha$ line modelling. We also plot results from a sample of LAEs analysed in a similar manner by modelling Ly $\alpha$ lines using shell models (Hashimoto et al. 2015), and a sample of LBGs, where the column density is derived from UV absorption line modelling (Wofford et al. 2013).

prime factor between these results, as a burst of star formation can have a much more destructive effect on a low mass galaxy than on a massive galaxy. The low outflow velocities and young ages are in agreement with a scenario where a short violent episode of star formation blows away a shell of gas at moderate velocity. In more massive galaxies, the star formation is less episodic, and the velocity needed to expell gas from the galaxy is higher. Therefore, one would expect to see a large scatter with on average very moderate velocities in low mass galaxies, and an increasing trend of outflow velocity with stellar mass at higher masses. We note that all but one velocity reported here are below the velocity of the lowest-mass bin in Bradshaw et al. (2013).

We used the expanding shell-model to fit the observed spectra with astonishing accuracy given the complexity of the spectral shape and the relatively few free parameters of the model. This finding is well aligned with previous studies (e.g. Verhamme et al. 2008; Schaerer et al. 2011; Hashimoto et al. 2015; Yang et al. 2016) who found the shell-model to represent also a good fit to the data.

In spite of this success, the shell-model is clearly a simplification of the complex velocity and density fields existing in highredshift galaxies and the physical meaning of the shell-model parameters is still unclear. Recently, Gronke \& Dijkstra (2016) found that the shell-model parameters do not match the ones of a more complex, multi-phase medium. This hints towards a more subtle conversion between the shell-model and the actual physical parameters. For example, Gronke \& Dijkstra (2016) showed that low column densities in shell models can reflect a medium consisting of optically thick clumps of gas with a low covering factor.

Independent of the question whether the parameters can be interpreted literally or are a more abstract quantity, we found several interesting correlations between them and other observed quantities. In particular, we found that the spectra can be reproduced best using lower neutral hydrogen column densities in shell models than in previous studies (Verhamme et al. 2008; Hashimoto et al. 2015) which studied brighter galaxies than our sample. This fact combined with the relative large Ly $\alpha$ escape fraction for most of our objects (the Ly $\alpha$ and LyC escape fractions are expected to correlate, see e.g., Yajima et al. 2014; Dijkstra et al. 2016), and the low mass (simulations suggest larger LyC escape fractions for lower mass galaxies, e.g., Paardekooper et al. 2015) makes our sample ideal candidates for LyC leaking galaxies.

When comparing properties as outflow velocity or column density to other studies, one has to be aware of differences in methods. Modelling Ly $\alpha$ profiles is very sensitive to neutral hydrogen, but as a large number of different properties of neutral hydrogen are involved, degeneracies arise naturally. For example, there are strong degeneracies between optical depth and EW, between $\sigma_{i}$ and the temperature of the gas. Perhaps the most important degeneracy arises between $z$ and a combination of $v_{\exp }$ and $N_{\mathrm{HI}}$, that is a difference in the systematic redshift can be compensated by changing the outflowing velocity and the column density or morphology or vice versa. The very consistent results of the Ly $\alpha$ modelling and the outflow velocity and column density determinations from other emission lines (see, Vanzella et al. 2016a) is encouraging. Results from relatively comparable samples of galaxies with different techniques strengthen the finding of low outflow velocities for faint LAEs (Erb et al. 2014; Trainor et al. 2015). Running a large number of parametrizations of the properties in combination with constraining the free parameters by other means helps to minimise the number of degeneracies. Gronke et al. (2015) showed that the column density, expansion velocity, and $\sigma$ are well recovered by these models, indicating that for the properties discussed here the influence of degeneracies is rather low. Other methods of determining the column density in outflows, for example through absorption line fitting (e.g. Wofford et al. 2013; Karman et al. 2014), are based on several assumptions such as temperature, metallicity, and electron density, which can lead to systematic differences. Although there is currently no evidence for these systematics, these concerns should be taken into consideration when comparing different techniques. We therefore caution the reader when we compared our column densities to those of Wofford et al. (2013).

The simplified modelling thus introduces additional uncertainties. As mentioned previously, if we are observing the galaxy through one of the holes of a patchy distribution, the modelled column density will be underestimated (Gronke \& Dijkstra 2016). Further, as star formation occurs stochastic in these low mass galaxies (e.g. Cloet-Osselaer et al. 2014; Hopkins et al. 2014; Maseda et al. 2014; Shen et al. 2014; Domínguez et al. 2015; Guo et al. 2016), it is expected that rather than a single shell, multiple shells are present from the multiple episodes of star formation. It is unclear how much this affects the galaxies studied here, as their ages indicate very young galaxies, preventing a large number of episodes of star formation.

The stochastic nature of low-mass galaxies poses another caveat, as we have here modelled the galaxies with a single exponentially decaying star formation history. The very young ages could be a result of a strong recent episode of star formation on top of a more evolved and older stellar population. However, for most of the galaxies, the non-detections in the Hawk-I observations limit a possible contribution of old stellar populations. At the moment, the available data are unfortunately insufficiently deep to directly observe the older population. This is because older populations dominate at longer wavelengths, for which data with similar depth and resolution are currently not achievable.

Although we are including the strongest emission lines in our SED-fitting, we are not including a nebular continuum. The best-fitting models match the observations relatively well in the 
restframe FUV, but the nebular emission is normally more dominant in the optical and FUV. By ignoring the nebular continuum, it is possible we slightly underestimate the ages, find slightly bluer UV continua, and slightly lower masses, as we have seen from a comparison of one galaxy within our sample (see Vanzella et al. 2016a). For this galaxy (SW-49), we included nebular continuum emission and a large number of absorption lines as described by Schaerer \& de Barros (2009). Including this, we find an increase of 0.4 dex in the stellar mass, a similar age, and an increase of 0.2 in $\beta$ compared to the values presented in Table 4 . These differences are within our error bars, but agree with the general trends discussed above. Therefore, the found differences are not significant enough to change trends or conclusions in this paper.

\section{Summary and conclusions}

In this article, we used deep IFU and broadband observations in combination with gravitational lensing to study the properties of intrinsically faint LAEs at $z>3$. We targeted two sides of the Frontier Fields cluster AS1063 for in total 10 hours of observation time. We determined spectroscopic redshifts for 172 galaxies in a $2 \operatorname{arcmin}^{2}$ central field of the cluster. Among these redshifts are 17 multiple redshift families, of which 11 did not have a redshift determination before. We extracted spectra for 14 LAEs, for which we observed in total 24 multiple images. These LAEs were corrected for lensing magnification by an updated gravitational lensing model, and are found to be among the intrinsically faintest observed at $z>3$. Without the help of lensing, the study of such a faint population of sources with absolute UV-magnitudes ranging from -19 to -14 would have been possible only with the next generation of telescopes, which are James Webb Space Telescope and European Extremely Large Telescope.

We modelled the Ly $\alpha$ line profiles using a spherically expanding gas shell model, and found that the narrow lines of these faint LAEs are best-fit low column densities. The line profiles are best fit with shells with low to moderate expansion velocities, which is in line with their low luminosities.

We obtained broadband photometry from the Hubble Frontiers Fields programme and the CLASH programme, and combined this with Hawk-I $K$-band and Spitzer IRAC data. We used the derived photometric catalogue to perform SED fitting on the LAEs, and obtained stellar masses and ages, the UV continuum slope, and $E(B-V)$. We found low stellar masses for these objects, significantly lower than masses reported for LAEs at any redshift before. In combination with the low stellar masses, these LAEs are characterised by young populations of tens of Myr, and low dust obscuration. These properties are very similar to the low-mass galaxies which are currently the main candidates for reionization, and provide therefore excellent analogues.

That these galaxies are recently formed and rapidly building up their stellar mass is clear from their SSFR, which is increased by an order of magnitude in our sample compared to other LAE samples. We found a comparable sSFR only in some very highredshift extreme emission line galaxies. This shows that these galaxies are starburst galaxies, and this rapidly forming nature might be characteristic for low mass galaxies at $z>3$. We note however, that quiescent low-mass galaxies would not be detected in our observations.

The UV continuum slope is very blue for these galaxies. We used the best-fitting SED model to derive $\beta$, and found that $\beta<-2$ for all galaxies with $M_{\star}<10^{8} M_{\odot}$. Such low values of $\beta$ are very rarely observed, and approach the theoretical limit of stellar population models. It has been argued, however, that for example top-heavy IMFs or Pop III stars can decrease $\beta$ further (e.g. Bouwens et al. 2010; Zackrisson et al. 2011), which could explain the even steeper slopes found for some of the LAEs discussed here.

We did not find any correlation between the expansion velocity and other quantities. The absence of a relation between SFR and $v_{\text {exp }}$ could be due to the stochastic nature of SF in low mass galaxies. This would increase the scatter such that any relation would be washed out in the mass range studied here. In agreement with this, the maximum velocities derived here are less than those found in more massive galaxies.

The observed decrease of $\beta$ with stellar mass, in combination with the previously found increase of occurence of Ly $\alpha$ emission with decreasing stellar mass, suggests that low mass galaxies might have been effective at leaking ionizing radiation. If the escape fraction of Lyman continuum photons follows these trends, this would confirm low mass galaxies as the main drivers of reionization. The candidacy for Lyman continuum leakage is strengthened by the narrowness of the Ly $\alpha$ lines, which indicates either a low column density or a low covering fraction. Both of these conditions are predicted to enable Lyman continuum leakage. Because these faint LAEs are probably good analogues to the galaxies responsible for reionization, it is important to determine if these galaxies are indeed leaking Lyman continuum radiation.

Acknowledgements. The authors thank the anonymous referee for constructive comments which helped improve the paper. The authors thank Ryan Trainor, Ryota Kawamata, and Kasper Schmidt for insightful comments on the manuscript. Based on observations made with the European Southern Observatory Very Large Telescope (ESO/VLT) at Cerro Paranal, under programme IDs 60.A-9345(A), 095.A-0653, and 095.A-0533. STScI is operated by the Association of Universities for Research in Astronomy, Inc. under NASA contract NAS 5-26555. C.G. acknowledges support by VILLUM FONDEN Young Investigator Programme through grant no. 10123. P.R., A.M. and M.N. acknowledge support from PRIN-INAF 2014 1.05.01.94.02 (PI M. Nonino). This research made use of APLpy, an open-source plotting package for Python hosted at http://aplpy.github.com.

\section{References}

Ahn, S.-H., Lee, H.-W., \& Lee, H. M. 2003, MNRAS, 340, 863 Amorín, R. O., Pérez-Montero, E., \& Vílchez, J. M. 2010, ApJ, 715, L128 Amorín, R., Sommariva, V., Castellano, M., et al. 2014, A\&A, 568, L8 Amorín, R., Pérez-Montero, E., Contini, T., et al. 2015, A\&A, 578, A105 Arnouts, S., Cristiani, S., Moscardini, L., et al. 1999, MNRAS, 310, 540 Arrigoni Battaia, F., Hennawi, J. F., Cantalupo, S., \& Prochaska, J. X. 2016, ApJ, 829,3

Atek, H., Siana, B., Scarlata, C., et al. 2011, ApJ, 743, 121

Atek, H., Kunth, D., Schaerer, D., et al. 2014, A\&A, 561, A89

Bacon, R., Accardo, M., Adjali, L., et al. 2012, The Messenger, 147, 4 Balestra, I., Vanzella, E., Rosati, P., et al. 2013, A\&A, 559, L9

Bertin, E. 2006, in Astronomical Data Analysis Software and Systems XV, eds. C. Gabriel, C. Arviset, D. Ponz, \& S. Enrique, ASP Conf. Ser., 351, 112

Bertin, E., Mellier, Y., Radovich, M., et al. 2002, in Astronomical Data Analysis Software and Systems XI, eds. D. A. Bohlender, D. Durand, \& T. H. Handley, ASP Conf. Ser., 281, 228

Bisigello, L., Caputi, K. I., Colina, L., et al. 2016, ApJS, 227, 19 Blanc, G. A., Adams, J. J., Gebhardt, K., et al. 2011, ApJ, 736, 31 Boone, F., Clément, B., Richard, J., et al. 2013, A\&A, 559, L1 Bouwens, R. J., Illingworth, G. D., Oesch, P. A., et al. 2010, ApJ, 708, L69 Bouwens, R. J., Illingworth, G. D., Oesch, P. A., et al. 2011, ApJ, 737, 90 Bouwens, R. J., Illingworth, G. D., Oesch, P. A., et al. 2014, ApJ, 793, 115 Bradshaw, E. J., Almaini, O., Hartley, W. G., et al. 2013, MNRAS, 433, 194 Brammer, G. B., Marchesini, D., Labbé, I., et al. 2016, ApJS, 226, 6 Bruzual, G., \& Charlot, S. 2003, MNRAS, 344, 1000

Calzetti, D., Kinney, A. L., \& Storchi-Bergmann, T. 1994, ApJ, 429, 582 Calzetti, D., Armus, L., Bohlin, R. C., et al. 2000, ApJ, 533, 682 Caminha, G. B., Karman, W., Rosati, P., et al. 2016a, A\&A, 595, A100 Caminha, G. B., Grillo, C., Rosati, P., et al. 2016b, A\&A, 587, A80 Caputi, K. I., Cirasuolo, M., Dunlop, J. S., et al. 2011, MNRAS, 413, 162 
Caputi, K. I., Ilbert, O., Laigle, C., et al. 2015, ApJ, 810, 73

Cardamone, C., Schawinski, K., Sarzi, M., et al. 2009, MNRAS, 399, 1191

Caruana, J., Bunker, A. J., Wilkins, S. M., et al. 2012, MNRAS, 427, 3055

Caruana, J., Bunker, A. J., Wilkins, S. M., et al. 2014, MNRAS, 443, 2831

Cassata, P., Le Fèvre, O., Garilli, B., et al. 2011, A\&A, 525, A143

Cassata, P., Tasca, L. A. M., Le Fèvre, O., et al. 2015, A\&A, 573, A24

Chisholm, J., Tremonti, C. A., Leitherer, C., et al. 2015, ApJ, 811, 149

Choudhury, T. R., Puchwein, E., Haehnelt, M. G., \& Bolton, J. S. 2015, MNRAS 452, 261

Cloet-Osselaer, A., De Rijcke, S., Vandenbroucke, B., et al. 2014, MNRAS, 442 2909

Cowie, L. L., Barger, A. J., \& Hu, E. M. 2011, ApJ, 738, 136

Curtis-Lake, E., McLure, R. J., Pearce, H. J., et al. 2012, MNRAS, 422, 1425

Dawson, S., Rhoads, J. E., Malhotra, S., et al. 2007, ApJ, 671, 1227

de Barros, S., Schaerer, D., \& Stark, D. P. 2014, A\&A, 563, A81

de Barros, S., Vanzella, E., Amorín, R., et al. 2016, A\&A, 585, A51

Díaz, C. G., Ryan-Weber, E. V., Cooke, J., Koyama, Y., \& Ouchi, M. 2015, MNRAS, 448, 1240

Diego, J. M., Broadhurst, T., Wong, J., et al. 2016, MNRAS, 459, 3447

Dijkstra, M. 2014, PASA, 31, e040

Dijkstra, M., Mesinger, A., \& Wyithe, J. S. B. 2011, MNRAS, 414, 2139

Dijkstra, M., Gronke, M., \& Venkatesan, A. 2016, ApJ, 828, 71

Domínguez, A., Siana, B., Brooks, A. M., et al. 2015, MNRAS, 451, 839

Dunlop, J. S., McLure, R. J., Robertson, B. E., et al. 2012, MNRAS, 420, 901

Earl, D. J., \& Deem, M. W. 2005, Phys. Chem. Chem. Phys., 7, 3910

Elíasdóttir, Á., Limousin, M., Richard, J., et al. 2007, ArXiv e-prints [arXiv:0710.5636]

Erb, D. K., Steidel, C. C., Trainor, R. F., et al. 2014, ApJ, 795, 33

Finkelstein, S. L., Papovich, C., Salmon, B., et al. 2012, ApJ, 756, 164

Finkelstein, S. L., Papovich, C., Dickinson, M., et al. 2013, Nature, 502, 524

Finkelstein, S. L., Ryan, Jr., R. E., Papovich, C., et al. 2015, ApJ, 810, 71

Foreman-Mackey, D., Hogg, D. W., Lang, D., \& Goodman, J. 2013, PASP, 125, 306

Forero-Romero, J. E., Yepes, G., Gottlöber, S., \& Prada, F. 2012, MNRAS, 419 952

Gardner, J. P., Mather, J. C., Clampin, M., et al. 2009, Astrophys. Space Sci. Proc., 10, 1

González, V., Labbé, I., Bouwens, R. J., et al. 2010, ApJ, 713, 115

Grillo, C., Karman, W., Suyu, S. H., et al. 2016, ApJ, 822, 78

Gronke, M., \& Dijkstra, M. 2014, MNRAS, 444, 1095

Gronke, M., \& Dijkstra, M. 2016, ApJ, 826, 14

Gronke, M., Bull, P., \& Dijkstra, M. 2015, ApJ, 812, 123

Gronwall, C., Ciardullo, R., Hickey, T., et al. 2007, ApJ, 667, 79

Guo, Y., Rafelski, M., Faber, S. M., et al. 2016, ApJ, 833, 37

Hagen, A., Ciardullo, R., Gronwall, C., et al. 2014, ApJ, 786, 59

Hashimoto, T., Verhamme, A., Ouchi, M., et al. 2015, ApJ, 812, 157

Hayes, M., Östlin, G., Duval, F., et al. 2014, ApJ, 782, 6

Henry, A. L., Martin, C. L., Dressler, A., Sawicki, M., \& McCarthy, P. 2012 ApJ, 744, 149

Henry, A., Scarlata, C., Martin, C. L., \& Erb, D. 2015, ApJ, 809, 19

Hopkins, P. F., Kereš, D., Oñorbe, J., et al. 2014, MNRAS, 445, 581

Ilbert, O., Arnouts, S., McCracken, H. J., et al. 2006, A\&A, 457, 841

Ilbert, O., Capak, P., Salvato, M., et al. 2009, ApJ, 690, 1236

Ilbert, O., McCracken, H. J., Le Fevre, O., et al. 2013, A\&A, 556, A55

Ilbert, O., Arnouts, S., Le Floc'h, E., et al. 2015, A\&A, 579, A2

Izotov, Y. I., Orlitová, I., Schaerer, D., et al. 2016, Nature, 529, 178

Jensen, H., Laursen, P., Mellema, G., et al. 2013, MNRAS, 428, 1366

Jiang, L., Finlator, K., Cohen, S. H., et al. 2016, ApJ, 816, 16

Johnson, T. L., Sharon, K., Bayliss, M. B., et al. 2014, ApJ, 797, 48

Jones, T. A., Ellis, R. S., Schenker, M. A., \& Stark, D. P. 2013, ApJ, 779, 52

Jullo, E., Kneib, J.-P., Limousin, M., et al. 2007, New J. Phys., 9, 447

Karman, W., Caputi, K. I., Trager, S. C., Almaini, O., \& Cirasuolo, M. 2014,

A\&A, 565, A5

Karman, W., Caputi, K. I., Grillo, C., et al. 2015, A\&A, 574, A11

Kashikawa, N., Shimasaku, K., Matsuda, Y., et al. 2011, ApJ, 734, 119

Kassiola, A., \& Kovner, I. 1993, ApJ, 417, 450

Kennicutt, Jr., R. C. 1998, ARA\&A, 36, 189

Kimm, T., \& Cen, R. 2014, ApJ, 788, 121

Kneib, J.-P., Ellis, R. S., Smail, I., Couch, W. J., \& Sharples, R. M. 1996, ApJ, 471,643

Koekemoer, A., Lotz, J. M., Coe, D., et al. 2016, ApJS, submitted

Kornei, K. A., Shapley, A. E., Erb, D. K., et al. 2010, ApJ, 711, 693
Kurk, J., Cimatti, A., Daddi, E., et al. 2013, A\&A, 549, A63

Laursen, P., Razoumov, A. O., \& Sommer-Larsen, J. 2009, ApJ, 696, 853

Laursen, P., Duval, F., \& Östlin, G. 2013, ApJ, 766, 124

Lotz, J. M., Koekemoer, A., Coe, D., et al. 2016, ApJ, submitted [arXiv: 1605.06567]

Ly, C., Malkan, M. A., Nagao, T., et al. 2014, ApJ, 780, 122

Mallery, R. P., Mobasher, B., Capak, P., et al. 2012, ApJ, 760, 128

Maseda, M. V., van der Wel, A., Rix, H.-W., et al. 2014, ApJ, 791, 17

Masters, D., \& Capak, P. 2011, PASP, 123, 638

Matthee, J., Sobral, D., Oteo, I., et al. 2016, MNRAS, 458, 449

McLinden, E. M., Rhoads, J. E., Malhotra, S., et al. 2014, MNRAS, 439, 446

Merlin, E., Fontana, A., Ferguson, H. C., et al. 2015, A\&A, 582, A15

Mesinger, A., Aykutalp, A., Vanzella, E., et al. 2015, MNRAS, 446, 566

Meurer, G. R., Heckman, T. M., \& Calzetti, D. 1999, ApJ, 521, 64

Monna, A., Seitz, S., Greisel, N., et al. 2014, MNRAS, 438, 1417

Nakajima, K., Ouchi, M., Shimasaku, K., et al. 2012, ApJ, 745, 12

Nilsson, K. K., Tapken, C., Møller, P., et al. 2009, A\&A, 498, 13

Oesch, P. A., van Dokkum, P. G., Illingworth, G. D., et al. 2015, ApJ, 804, L30

Oesch, P. A., Brammer, G., van Dokkum, P. G., et al. 2016, ApJ, 819, 129

Ono, Y., Ouchi, M., Shimasaku, K., et al. 2010, MNRAS, 402, 1580

Ono, Y., Ouchi, M., Mobasher, B., et al. 2012, ApJ, 744, 83

Ouchi, M., Shimasaku, K., Akiyama, M., et al. 2008, ApJS, 176, 301

Ouchi, M., Shimasaku, K., Furusawa, H., et al. 2010, ApJ, 723, 869

Oyarzún, G. A., Blanc, G. A., González, V., et al. 2016, ApJ, 821, L14

Paardekooper, J.-P., Khochfar, S., \& Dalla Vecchia, C. 2015, MNRAS, 451, 2544

Pénin, A., Cuby, J.-G., Clément, B., et al. 2015, A\&A, 577, A74

Pentericci, L., Fontana, A., Vanzella, E., et al. 2011, ApJ, 743, 132

Pentericci, L., Vanzella, E., Fontana, A., et al. 2014, ApJ, 793, 113

Postman, M., Coe, D., Benítez, N., et al. 2012, ApJS, 199, 25

Rauch, M., Haehnelt, M., Bunker, A., et al. 2008, ApJ, 681, 856

Richard, J., Jauzac, M., Limousin, M., et al. 2014, MNRAS, 444, 268

Salmon, B., Papovich, C., Finkelstein, S. L., et al. 2015, ApJ, 799, 183

Sawicki, M., Lemaux, B. C., Guhathakurta, P., et al. 2008, ApJ, 687, 884

Schaerer, D., \& de Barros, S. 2009, A\&A, 502, 423

Schaerer, D., Hayes, M., Verhamme, A., \& Teyssier, R. 2011, A\&A, 531, A12

Schenker, M. A., Stark, D. P., Ellis, R. S., et al. 2012, ApJ, 744, 179

Schenker, M. A., Robertson, B. E., Ellis, R. S., et al. 2013, ApJ, 768, 196

Schmidt, K. B., Treu, T., Bradač, M., et al. 2016, ApJ, 818, 38

Shapley, A. E., Steidel, C. C., Adelberger, K. L., et al. 2001, ApJ, 562, 95

Shapley, A. E., Steidel, C. C., Pettini, M., \& Adelberger, K. L. 2003, ApJ, 588, 65

Sharma, M., Theuns, T., Frenk, C., et al. 2016, MNRAS, 458, L94

Shen, S., Madau, P., Conroy, C., Governato, F., \& Mayer, L. 2014, ApJ, 792, 99

Shimasaku, K., Kashikawa, N., Doi, M., et al. 2006, PASJ, 58, 313

Speagle, J. S., Steinhardt, C. L., Capak, P. L., \& Silverman, J. D. 2014, ApJS, 214,15

Stark, D. P., Ellis, R. S., Chiu, K., Ouchi, M., \& Bunker, A. 2010, MNRAS, 408, 1628

Stark, D. P., Schenker, M. A., Ellis, R., et al. 2013, ApJ, 763, 129

Steidel, C. C., Giavalisco, M., Pettini, M., Dickinson, M., \& Adelberger, K. L. 1996, ApJ, 462, L17

Steidel, C. C., Adelberger, K. L., Shapley, A. E., et al. 2003, ApJ, 592, 728

Suyu, S. H., Marshall, P. J., Auger, M. W., et al. 2010, ApJ, 711, 201

Tasca, L. A. M., Le Fèvre, O., Hathi, N. P., et al. 2015, A\&A, 581, A54

Trainor, R. F., Steidel, C. C., Strom, A. L., \& Rudie, G. C. 2015, ApJ, 809, 89

Treu, T., Schmidt, K. B., Brammer, G. B., et al. 2015, ApJ, 812, 114

van der Wel, A., Straughn, A. N., Rix, H.-W., et al. 2011, ApJ, 742, 111

Vanzella, E., De Barros, S., Cupani, G., et al. 2016a, ApJ, 821, L27

Vanzella, E., de Barros, S., Vasei, K., et al. 2016b, ApJ, 825, 41

Verhamme, A., Schaerer, D., \& Maselli, A. 2006, A\&A, 460, 397

Verhamme, A., Schaerer, D., Atek, H., \& Tapken, C. 2008, A\&A, 491, 89

Verhamme, A., Orlitová, I., Schaerer, D., \& Hayes, M. 2015, A\&A, 578, A7

Weiner, B. J., Coil, A. L., Prochaska, J. X., et al. 2009, ApJ, 692, 187

Whitaker, K. E., Franx, M., Leja, J., et al. 2014, ApJ, 795, 104

Wise, J. H., Demchenko, V. G., Halicek, M. T., et al. 2014, MNRAS, 442, 2560

Wofford, A., Leitherer, C., \& Salzer, J. 2013, ApJ, 765, 118

Yajima, H., Li, Y., Zhu, Q., et al. 2014, MNRAS, 440, 776

Yang, H., Malhotra, S., Gronke, M., et al. 2016, ApJ, 820, 130

Yuma, S., Ohta, K., Yabe, K., et al. 2010, ApJ, 720, 1016

Zackrisson, E., Rydberg, C.-E., Schaerer, D., Östlin, G., \& Tuli, M. 2011, ApJ, 740, 13

Zackrisson, E., Inoue, A. K., \& Jensen, H. 2013, ApJ, 777, 39 


\section{Appendix A: Redshifts in AS1063}

As described in Sect. 2, we extracted spectra for all sources for which we; 1 ) extracted a location in the spectrally stacked MUSE image using SEXTRACTOR; 2) found emission lines in the MUSE datacube through visual inspection; 3) multiple images of a source were expected from the lensing model; 4) galaxies with a $M_{F 814 W}<23.5$. We repeated this analysis on the already published data of the south-western half of the cluster (Paper I), and added 13 cluster galaxies with spectroscopic redshift, two multiply lensed galaxies described by $\mathrm{C} 16$, and one new multiply lensed galaxy described in the text to the catalogue presented in Paper I.

We determined redshifts in the same way as described by Grillo et al. (2016), where redshifts were independently confirmed by two authors. Finetuning of the fourth decimal was done by a cross correlation with a template using SPECPRO (Masters \& Capak 2011), where we added an additional higherresolution GMASS template (Kurk et al. 2013). Visual confirmation of the finetuning was performed for all spectra, and, if necessary, redshifts were slightly adjusted in case of residual offsets. We note that the quality flags used here follow those used in Grillo et al. (2016), and differ from those used in Paper I, decreasing in quality from 4 to 1 . A QF of 4 is a highly secure redshift $(\delta z<0.0004)), \mathrm{QF}=3$ is a secure redshift $(\delta z<0.001))$, $\mathrm{QF}=2$ is an uncertain redshift $(\delta z<0.01))$, while $\mathrm{QF}=1$ indicates a tentative redshift. If the redshift is based on emission lines, we use $\mathrm{QF}=92$ in case of a doublet or a clear line profile, while a single emission line without a characteristic profile is assigned a $\mathrm{QF}=91$. We labelled all sources according to the region of detection (NE or SW) for clarity, keeping the IDs assigned previously by Paper I or C16.

We compared our redshifts to the release of the Grism LensAmplified Survey from Space survey (GLASS; Treu et al. 2015; Schmidt et al. 2016). In general the redshifts agree reasonably well, see Fig. A.1, and we find disagreeing redshifts for only 12 galaxies which have low quality flags in GLASS. We note that the disagreeing redshifts might be largely due to an incorrect cross matching, where they find lensed background galaxies, while we indentified the lensing cluster members. In addition, we determined redshifts for over 50 more galaxies in the inner part of the cluster, while six galaxies with redshifts from GLASS are not identified in our catalogue. We note that the area of GLASS extends much further from the centre of the cluster and the spectroscopy is performed in the NIR, making the two catalogues complementary. Unfortunately, only one of the LAE candidates at $z>6.5$ proposed by Schmidt et al. (2016) falls within the observed area, and we do not detect any emission lines at this position. This non-detection strenghtens the Ly $\alpha$ identification proposed by Schmidt et al. (2016).
Table A.1. Redshifts NE of AS1063.

\begin{tabular}{|c|c|c|c|c|c|}
\hline ID & $\begin{array}{c}\text { RA } \\
(\mathrm{J} 2000)\end{array}$ & $\begin{array}{c}\text { Dec } \\
(\mathrm{J} 2000)\end{array}$ & $z$ & $\mathrm{QF}$ & EL \\
\hline NE-1 & 342.18700 & -44.52617 & 0.0000 & 2 & $\mathrm{~N}$ \\
\hline NE-2 & 342.19297 & -44.52816 & 0.0000 & 2 & $\mathrm{~N}$ \\
\hline SW-58 & 342.17277 & -44.54545 & 0.0000 & 3 & $\mathrm{~N}$ \\
\hline SW-35 & 342.18081 & -44.54647 & 0.1530 & 4 & $\mathrm{Y}$ \\
\hline SW-36 & 342.17165 & -44.52896 & 0.1602 & 4 & $\mathrm{Y}$ \\
\hline NE-3 & 342.19667 & -44.52260 & 0.2409 & 4 & $\mathrm{Y}$ \\
\hline NE-4 & 342.18910 & -44.52116 & 0.2637 & 3 & $\mathrm{Y}$ \\
\hline NE-5 & 342.18922 & -44.52118 & 0.2637 & 4 & $\mathrm{Y}$ \\
\hline NE-6 & 342.18969 & -44.52006 & 0.2641 & 4 & $\mathrm{Y}$ \\
\hline SW-1 & 342.16708 & -44.53469 & 0.3263 & 4 & $\mathrm{~N}$ \\
\hline NE-7 & 342.17937 & -44.52792 & 0.3275 & 4 & $\mathrm{~N}$ \\
\hline SW-73 & 342.16877 & -44.53399 & 0.3278 & 1 & $\mathrm{~N}$ \\
\hline NE-8 & 342.18726 & -44.52038 & 0.3279 & 1 & $\mathrm{~N}$ \\
\hline NE-95 & 342.19711 & -44.52467 & 0.3300 & 2 & $\mathrm{~N}$ \\
\hline NE-9 & 342.19795 & -44.52782 & 0.3320 & 2 & $\mathrm{~N}$ \\
\hline NE-10 & 342.18430 & -44.52807 & 0.3330 & 4 & $\mathrm{~N}$ \\
\hline SW-2 & 342.17691 & -44.53408 & 0.3343 & 4 & $\mathrm{~N}$ \\
\hline NE-11 & 342.18657 & -44.53028 & 0.3347 & 3 & $\mathrm{Y}$ \\
\hline SW-3 & 342.18579 & -44.53454 & 0.3348 & 4 & $\mathrm{~N}$ \\
\hline SW-4 & 342.17449 & -44.54621 & 0.3350 & 3 & $\mathrm{~N}$ \\
\hline SW-64 & 342.18556 & -44.53859 & 0.3350 & 1 & $\mathrm{~N}$ \\
\hline SW-5 & 342.17085 & -44.53587 & 0.3360 & 4 & $\mathrm{Y}$ \\
\hline SW-6 & 342.17546 & -44.53542 & 0.3365 & 4 & $\mathrm{Y}$ \\
\hline NE-12 & 342.19189 & -44.52967 & 0.3366 & 3 & $\mathrm{~N}$ \\
\hline NE-13 & 342.19207 & -44.52283 & 0.3368 & 1 & $\mathrm{~N}$ \\
\hline SW-9 & 342.17704 & -44.53694 & 0.3368 & 4 & $\mathrm{Y}$ \\
\hline SW-7 & 342.17158 & -44.53948 & 0.3370 & 3 & $\mathrm{~N}$ \\
\hline NE-14 & 342.19366 & -44.51850 & 0.3378 & 4 & $\mathrm{~N}$ \\
\hline NE-15 & 342.18901 & -44.52470 & 0.3382 & 4 & $\mathrm{~N}$ \\
\hline SW-8 & 342.18694 & -44.53536 & 0.3383 & 4 & $\mathrm{~N}$ \\
\hline SW-60 & 342.17993 & -44.53560 & 0.3384 & 2 & $\mathrm{~N}$ \\
\hline NE-16 & 342.18419 & -44.52030 & 0.3386 & 3 & $\mathrm{~N}$ \\
\hline NE-17 & 342.18812 & -44.53281 & 0.3387 & 3 & $\mathrm{~N}$ \\
\hline NE-18 & 342.18663 & -44.52248 & 0.3388 & 4 & $\mathrm{~N}$ \\
\hline SW-71 & 342.18116 & -44.53788 & 0.3388 & 2 & $\mathrm{~N}$ \\
\hline NE-19 & 342.17960 & -44.52307 & 0.3390 & 4 & $\mathrm{Y}$ \\
\hline NE-20 & 342.19327 & -44.52413 & 0.3390 & 2 & $\mathrm{~N}$ \\
\hline SW-61 & 342.17462 & -44.53699 & 0.3390 & 2 & $\mathrm{~N}$ \\
\hline NE-21 & 342.18917 & -44.52368 & 0.3397 & 2 & $\mathrm{~N}$ \\
\hline SW-72 & 342.17526 & -44.53141 & 0.3398 & 1 & $\mathrm{~N}$ \\
\hline NE-22 & 342.19589 & -44.52368 & 0.3400 & 1 & $\mathrm{~N}$ \\
\hline NE-23 & 342.19547 & -44.53244 & 0.3401 & 3 & $\mathrm{~N}$ \\
\hline NE-24 & 342.18295 & -44.52495 & 0.3406 & 3 & $\mathrm{~N}$ \\
\hline NE-25 & 342.19517 & -44.52925 & 0.3410 & 1 & $\mathrm{~N}$ \\
\hline SW-10 & 342.17490 & -44.53412 & 0.3410 & 4 & $\mathrm{~N}$ \\
\hline SW-11 & 342.16657 & -44.53483 & 0.3420 & 4 & $\mathrm{~N}$ \\
\hline NE-26 & 342.19328 & -44.51782 & 0.3422 & 4 & $\mathrm{~N}$ \\
\hline NE-27 & 342.19269 & -44.51492 & 0.3424 & 3 & $\mathrm{~N}$ \\
\hline NE-28 & 342.19421 & -44.52209 & 0.3424 & 4 & $\mathrm{Y}$ \\
\hline
\end{tabular}

Notes. Sources are labelled using their position with regard to the pointing in which they were found, i.e. IDs with SW- (NE-) are found in the south-western (north-eastern) half of the MUSE observations. We crossreferenced the sources with a multiple image detection in Table B.1 with the label MI-. Quality flags (QF) are 4 very certain redshift $(\delta z<0.003)$; 3 certain redshift $(\delta z<0.001) ; 2$ good redshift $(\delta z<0.01), 1$ tentative redshift. The $\mathrm{QF}>90$ are based on a single emission line, where 93 illustrates that the profile of the line is asymmetrical, 92 shows it is a doublet, and 91 has only a single emission line detection, without any significant features in the profile. In the last column, we show if we detect emission lines $(E L=Y)$ or not $(E L=N)$. 
Table A.1. continued.

\begin{tabular}{|c|c|c|c|c|c|}
\hline ID & $\begin{array}{c}\text { RA } \\
(\mathrm{J} 2000)\end{array}$ & $\begin{array}{c}\text { Dec } \\
(\mathrm{J} 2000)\end{array}$ & $z$ & $\mathrm{QF}$ & EL \\
\hline NE-29 & 342.18844 & -44.51775 & 0.3427 & 4 & $\mathrm{Y}$ \\
\hline NE-30 & 342.18902 & -44.51923 & 0.3427 & 4 & $\mathrm{Y}$ \\
\hline NE-31 & 342.19019 & -44.51652 & 0.3428 & 4 & $\mathrm{Y}$ \\
\hline NE-32 & 342.19293 & -44.52207 & 0.3431 & 4 & $\mathrm{Y}$ \\
\hline NE-33 & 342.20085 & -44.52723 & 0.3431 & 2 & $\mathrm{~N}$ \\
\hline SW-12 & 342.18205 & -44.54034 & 0.3435 & 4 & $\mathrm{~N}$ \\
\hline NE-34 & 342.20017 & -44.52722 & 0.3436 & 3 & $\mathrm{~N}$ \\
\hline NE-35 & 342.18539 & -44.51864 & 0.3440 & 4 & $\mathrm{~N}$ \\
\hline NE-36 & 342.18571 & -44.52602 & 0.3440 & 3 & $\mathrm{~N}$ \\
\hline SW-13 & 342.18940 & -44.53689 & 0.3440 & 4 & $\mathrm{~N}$ \\
\hline NE-37 & 342.17724 & -44.52502 & 0.3446 & 2 & $\mathrm{~N}$ \\
\hline NE-38 & 342.19270 & -44.51976 & 0.3447 & 3 & $\mathrm{~N}$ \\
\hline NE-39 & 342.19333 & -44.52642 & 0.3448 & 4 & $\mathrm{~N}$ \\
\hline SW-15 & 342.18685 & -44.53389 & 0.3450 & 3 & $\mathrm{~N}$ \\
\hline NE-40 & 342.20125 & -44.52404 & 0.3453 & 2 & $\mathrm{~N}$ \\
\hline NE-41 & 342.19123 & -44.53513 & 0.3454 & 3 & $\mathrm{~N}$ \\
\hline NE-42 & 342.19550 & -44.52599 & 0.3455 & 4 & $\mathrm{~N}$ \\
\hline SW-14 & 342.17447 & -44.52899 & 0.3457 & 4 & $\mathrm{~N}$ \\
\hline NE-43 & 342.20122 & -44.52067 & 0.3459 & 3 & $\mathrm{~N}$ \\
\hline NE-44 & 342.19577 & -44.52415 & 0.3460 & 1 & $\mathrm{~N}$ \\
\hline NE-45 & 342.18453 & -44.52931 & 0.3463 & 3 & $\mathrm{~N}$ \\
\hline NE-46 & 342.17794 & -44.52407 & 0.3464 & 4 & $\mathrm{~N}$ \\
\hline NE-47 & 342.18111 & -44.52924 & 0.3464 & 4 & $\mathrm{~N}$ \\
\hline SW-17 & 342.17692 & -44.53744 & 0.3465 & 3 & $\mathrm{~N}$ \\
\hline NE-48 & 342.19001 & -44.53450 & 0.3470 & 1 & $\mathrm{~N}$ \\
\hline NE-49 & 342.19110 & -44.53295 & 0.3470 & 1 & $\mathrm{~N}$ \\
\hline SW-16 & 342.17648 & -44.53363 & 0.3470 & 4 & $\mathrm{~N}$ \\
\hline NE-50 & 342.18569 & -44.53051 & 0.3471 & 3 & $\mathrm{~N}$ \\
\hline NE-51 & 342.18663 & -44.53109 & 0.3475 & 4 & $\mathrm{~N}$ \\
\hline SW-21 & 342.17178 & -44.54054 & 0.3475 & 4 & $\mathrm{~N}$ \\
\hline SW-20 & 342.18898 & -44.54037 & 0.3476 & 4 & $\mathrm{~N}$ \\
\hline NE-52 & 342.18368 & -44.53056 & 0.3478 & 4 & $\mathrm{~N}$ \\
\hline NE-53 & 342.18916 & -44.52954 & 0.3479 & 4 & $\mathrm{~N}$ \\
\hline NE-54 & 342.18293 & -44.53046 & 0.3480 & 4 & $\mathrm{~N}$ \\
\hline NE-55 & 342.18857 & -44.52671 & 0.3480 & 2 & $\mathrm{~N}$ \\
\hline NE-56 & 342.19656 & -44.51974 & 0.3480 & 1 & $\mathrm{~N}$ \\
\hline NE-57 & 342.18256 & -44.52688 & 0.3484 & 4 & $\mathrm{~N}$ \\
\hline NE-58 & 342.19528 & -44.53483 & 0.3485 & 2 & $\mathrm{~N}$ \\
\hline SW-22 & 342.17903 & -44.53276 & 0.3485 & 3 & $\mathrm{~N}$ \\
\hline SW-23 & 342.16985 & -44.53555 & 0.3485 & 4 & $\mathrm{~N}$ \\
\hline NE-59 & 342.19159 & -44.53335 & 0.3486 & 4 & $\mathrm{~N}$ \\
\hline SW-18 & 342.18303 & -44.53099 & 0.3488 & 3 & $\mathrm{~N}$ \\
\hline SW-25 & 342.18671 & -44.54074 & 0.3488 & 4 & $\mathrm{~N}$ \\
\hline NE-60 & 342.18669 & -44.52061 & 0.3489 & 2 & $\mathrm{~N}$ \\
\hline SW-19 & 342.18550 & -44.53305 & 0.3489 & 4 & $\mathrm{~N}$ \\
\hline NE-61 & 342.18564 & -44.53124 & 0.3490 & 3 & $\mathrm{~N}$ \\
\hline SW-63 & 342.18488 & -44.54065 & 0.3491 & 2 & $\mathrm{~N}$ \\
\hline NE-62 & 342.18815 & -44.52973 & 0.3493 & 4 & $\mathrm{~N}$ \\
\hline SW-24 & 342.16826 & -44.53658 & 0.3493 & 4 & $\mathrm{~N}$ \\
\hline NE-63 & 342.18813 & -44.52597 & 0.3494 & 4 & $\mathrm{~N}$ \\
\hline SW-55 & 342.16909 & -44.54040 & 0.3494 & 2 & $\mathrm{~N}$ \\
\hline SW-56 & 342.17217 & -44.54314 & 0.3494 & 2 & $\mathrm{~N}$ \\
\hline NE-64 & 342.20508 & -44.52591 & 0.3495 & 2 & $\mathrm{~N}$ \\
\hline NE-65 & 342.18907 & -44.52645 & 0.3498 & 2 & $\mathrm{~N}$ \\
\hline SW-26 & 342.18307 & -44.53307 & 0.3498 & 4 & $\mathrm{~N}$ \\
\hline SW-28 & 342.18196 & -44.53857 & 0.3502 & 3 & $\mathrm{~N}$ \\
\hline SW-62 & 342.18197 & -44.53858 & 0.3502 & 3 & $\mathrm{~N}$ \\
\hline
\end{tabular}

Table A.1. continued.

\begin{tabular}{|c|c|c|c|c|c|}
\hline ID & $\begin{array}{c}\text { RA } \\
(\mathrm{J} 2000)\end{array}$ & $\begin{array}{c}\text { Dec } \\
(\mathrm{J} 2000)\end{array}$ & $z$ & $\mathrm{QF}$ & EL \\
\hline NE-66 & 342.20420 & -44.52523 & 0.3503 & 4 & $\mathrm{~N}$ \\
\hline NE-67 & 342.18406 & -44.52694 & 0.3504 & 4 & $\mathrm{~N}$ \\
\hline SW-27 & 342.17793 & -44.53239 & 0.3505 & 4 & $\mathrm{~N}$ \\
\hline NE-68 & 342.17961 & -44.52858 & 0.3510 & 2 & $\mathrm{~N}$ \\
\hline NE-69 & 342.19728 & -44.52325 & 0.3510 & 4 & $\mathrm{Y}$ \\
\hline NE-70 & 342.19534 & -44.53490 & 0.3514 & 2 & $\mathrm{~N}$ \\
\hline NE-71 & 342.17891 & -44.52472 & 0.3515 & 3 & $\mathrm{~N}$ \\
\hline NE-72 & 342.19462 & -44.53228 & 0.3520 & 2 & $\mathrm{~N}$ \\
\hline SW-30 & 342.17872 & -44.54657 & 0.3525 & 3 & $\mathrm{~N}$ \\
\hline SW-29 & 342.18452 & -44.54318 & 0.3526 & 4 & $\mathrm{~N}$ \\
\hline NE-73 & 342.18353 & -44.53011 & 0.3530 & 4 & $\mathrm{~N}$ \\
\hline NE-74 & 342.19002 & -44.52413 & 0.3530 & 2 & $\mathrm{~N}$ \\
\hline NE-75 & 342.20030 & -44.52519 & 0.3530 & 4 & $\mathrm{~N}$ \\
\hline NE-76 & 342.19137 & -44.53433 & 0.3532 & 4 & $\mathrm{~N}$ \\
\hline SW-57 & 342.18324 & -44.54386 & 0.3532 & 2 & $\mathrm{~N}$ \\
\hline SW-77 & 342.18533 & -44.53412 & 0.3532 & 3 & $\mathrm{~N}$ \\
\hline NE-77 & 342.17911 & -44.52866 & 0.3533 & 1 & $\mathrm{~N}$ \\
\hline SW-31 & 342.18436 & -44.53617 & 0.3535 & 3 & $\mathrm{~N}$ \\
\hline NE-78 & 342.18674 & -44.52786 & 0.3550 & 4 & $\mathrm{~N}$ \\
\hline SW-32 & 342.16265 & -44.53809 & 0.3550 & 3 & $\mathrm{~N}$ \\
\hline SW-33 & 342.16044 & -44.53896 & 0.3553 & 4 & $\mathrm{~N}$ \\
\hline NE-79 & 342.19670 & -44.52909 & 0.3572 & 3 & $\mathrm{~N}$ \\
\hline SW-34 & 342.17366 & -44.53278 & 0.3588 & 4 & $\mathrm{~N}$ \\
\hline NE-80 & 342.19823 & -44.52741 & 0.3606 & 3 & $\mathrm{~N}$ \\
\hline NE-81 & 342.20442 & -44.52459 & 0.3799 & 4 & $\mathrm{Y}$ \\
\hline NE-82 & 342.19539 & -44.51676 & 0.3940 & 1 & $\mathrm{~N}$ \\
\hline NE-83 & 342.20296 & -44.52716 & 0.4382 & 4 & $\mathrm{Y}$ \\
\hline NE-84 & 342.20568 & -44.52325 & 0.4386 & 4 & $\mathrm{Y}$ \\
\hline SW-37 & 342.16658 & -44.54021 & 0.4582 & 4 & $\mathrm{Y}$ \\
\hline NE-85 & 342.18576 & -44.52405 & 0.4845 & 4 & $\mathrm{Y}$ \\
\hline SW-39 & 342.18870 & -44.53788 & 0.6108 & 4 & $\mathrm{Y}$ \\
\hline SW-38 & 342.17403 & -44.53244 & 0.6111 & 4 & $\mathrm{Y}$ \\
\hline SW-40 & 342.18442 & -44.53957 & 0.6518 & 4 & $\mathrm{Y}$ \\
\hline SW-41 & 342.17925 & -44.54219 & 0.6980 & 4 & Y \\
\hline NE-86 & 342.20357 & -44.52496 & 0.7048 & 3 & $\mathrm{Y}$ \\
\hline SW-42 & 342.18014 & -44.54407 & 0.7145 & 4 & $\mathrm{Y}$ \\
\hline $\begin{array}{l}\text { NE-87a/ } \\
\text { MI-87a }\end{array}$ & 342.18429 & -44.52529 & 0.7287 & 4 & $\mathrm{Y}$ \\
\hline $\begin{array}{l}\text { NE-87b/ } \\
\text { MI-87b }\end{array}$ & 342.18894 & -44.52864 & 0.7287 & 4 & $\mathrm{Y}$ \\
\hline $\begin{array}{l}\text { NE-87c/ } \\
\text { MI-87c }\end{array}$ & 342.19010 & -44.53010 & 0.7287 & 4 & $\mathrm{Y}$ \\
\hline $\begin{array}{l}\text { SW-43a/ } \\
\text { MI-7c }\end{array}$ & 342.17209 & -44.53052 & 1.0350 & 4 & $\mathrm{Y}$ \\
\hline $\begin{array}{l}\text { SW-43b/ } \\
\text { MI-7b }\end{array}$ & 342.17597 & -44.53615 & 1.0350 & 4 & $\mathrm{Y}$ \\
\hline $\begin{array}{c}\text { SW-43c/ } \\
\text { MI-7a }\end{array}$ & 342.18069 & -44.53866 & 1.0350 & 4 & $\mathrm{Y}$ \\
\hline $\begin{array}{l}\text { NE-88a/ } \\
\text { MI-2c }\end{array}$ & 342.18642 & -44.52116 & 1.2277 & 4 & $\mathrm{Y}$ \\
\hline $\begin{array}{l}\text { NE-88b/ } \\
\text { MI-2a }\end{array}$ & 342.19588 & -44.52895 & 1.2278 & 4 & $\mathrm{Y}$ \\
\hline $\begin{array}{l}\text { NE-88c/ } \\
\text { MI- } 2 b\end{array}$ & 342.19450 & -44.52698 & 1.2279 & 4 & $\mathrm{Y}$ \\
\hline $\begin{array}{l}\text { NE-88d/ } \\
\text { MI-2d }\end{array}$ & 342.19520 & -44.52786 & 1.2279 & 4 & $\mathrm{Y}$ \\
\hline $\begin{array}{l}\text { NE-102a/ } \\
\text { MI-13a }\end{array}$ & 342.19369 & -44.53014 & 1.2583 & 91 & $\mathrm{Y}$ \\
\hline NE-102b/ & 342.19331 & -44.52942 & 1.2583 & 91 & Y \\
\hline
\end{tabular}


W. Karman et al.: Properties of low luminosity LAEs at $z>3$

Table A.1. continued.

\begin{tabular}{|c|c|c|c|c|c|}
\hline ID & $\begin{array}{c}\text { RA } \\
(\mathrm{J} 2000)\end{array}$ & $\begin{array}{c}\text { Dec } \\
(\mathrm{J} 2000)\end{array}$ & $z$ & $\mathrm{QF}$ & EL \\
\hline MI-13b & & & & & \\
\hline $\begin{array}{l}\text { NE-89/ } \\
\text { MI-3a }\end{array}$ & 342.19271 & -44.53119 & 1.2592 & 4 & $\mathrm{Y}$ \\
\hline $\begin{array}{l}\text { NE-89/ } \\
\text { MI-3b }\end{array}$ & 342.19213 & -44.52983 & 1.2593 & 4 & Y \\
\hline SW-44 & 342.17253 & -44.54128 & 1.2690 & 3 & Y \\
\hline SW-45 & 342.17552 & -44.54558 & 1.2690 & 92 & Y \\
\hline $\begin{array}{l}\text { NE-90a/ } \\
\text { MI-4c }\end{array}$ & 342.17921 & -44.52359 & 1.3972 & 92 & Y \\
\hline $\begin{array}{l}\text { NE-90b/ } \\
\text { MI-4b }\end{array}$ & 342.18783 & -44.52731 & 1.3972 & 92 & $\mathrm{Y}$ \\
\hline $\begin{array}{l}\text { NE-90c/ } \\
\text { MI-4a }\end{array}$ & 342.19317 & -44.53653 & 1.3972 & 92 & Y \\
\hline $\begin{array}{c}\text { SW-46a/ } \\
\text { MI-6a }\end{array}$ & 342.18843 & -44.53997 & 1.4285 & 4 & Y \\
\hline $\begin{array}{l}\text { SW-46b/ } \\
\text { MI-6b }\end{array}$ & 342.17583 & -44.53258 & 1.4285 & 4 & $\mathrm{Y}$ \\
\hline $\begin{array}{l}\text { SW-46c/ } \\
\text { MI-6c }\end{array}$ & 342.17409 & -44.52844 & 1.4285 & 4 & $\mathrm{Y}$ \\
\hline SW-47 & 342.17699 & -44.54633 & 1.4770 & 92 & Y \\
\hline $\begin{array}{c}\text { SW-69a/ } \\
\text { MI-8a }\end{array}$ & 342.18186 & -44.54050 & 1.8370 & 92 & $\mathrm{Y}$ \\
\hline $\begin{array}{l}\text { SW-69b/ } \\
\text { MI-8b }\end{array}$ & 342.17424 & -44.53711 & 1.8370 & 92 & Y \\
\hline SW-48 & 342.16135 & -44.53835 & 2.5770 & 91 & Y \\
\hline $\begin{array}{l}\text { NE-91/ } \\
\text { MI-91a }\end{array}$ & 342.19238 & -44.52505 & 2.9760 & 91 & $\mathrm{Y}$ \\
\hline NE-92 & 342.19171 & -44.53052 & 3.0600 & 1 & $\mathrm{~N}$ \\
\hline SW-50 & 342.16225 & -44.53829 & 3.1160 & 4 & Y \\
\hline $\begin{array}{l}\text { SW-68a/ } \\
\text { MI-20a }\end{array}$ & 342.18745 & -44.53869 & 3.1166 & 4 & $\mathrm{Y}$ \\
\hline $\begin{array}{l}\text { SW-68b/ } \\
\text { MI-20b }\end{array}$ & 342.17886 & -44.53587 & 3.1166 & 4 & $\mathrm{Y}$ \\
\hline $\begin{array}{l}\text { SW-49a } \\
\text { MI-11a }\end{array}$ & 342.17505 & -44.54102 & 3.1169 & 4 & $\mathrm{Y}$ \\
\hline $\begin{array}{l}\text { SW-49b/ } \\
\text { MI-11b }\end{array}$ & 342.17318 & -44.54000 & 3.1169 & 4 & Y \\
\hline $\begin{array}{l}\text { NE-93a/ } \\
\text { MI-93a }\end{array}$ & 342.18283 & -44.52028 & 3.169 & 92 & Y \\
\hline $\begin{array}{l}\text { NE-93b/ } \\
\text { MI-93b }\end{array}$ & 342.19196 & -44.52409 & 3.169 & 92 & $\mathrm{Y}$ \\
\hline SW-51 & 342.17402 & -44.54124 & 3.2275 & 4 & $\mathrm{Y}$ \\
\hline $\begin{array}{l}\text { NE-94a/ } \\
\text { MI-94a }\end{array}$ & 342.18935 & -44.51871 & 3.2857 & 92 & Y \\
\hline $\begin{array}{l}\text { NE-94b/ } \\
\text { MI-94b }\end{array}$ & 342.19615 & -44.52292 & 3.2857 & 92 & Y \\
\hline NE-96 & 342.19709 & -44.52483 & 3.4514 & 92 & $\mathrm{Y}$ \\
\hline SW-70a/ & 342.18586 & -44.53883 & 3.6063 & 2 & $\mathrm{~N}$ \\
\hline
\end{tabular}

Table A.1. continued.

\begin{tabular}{cccccc}
\hline \hline ID & $\begin{array}{c}\text { RA } \\
(\mathrm{J} 2000)\end{array}$ & $\begin{array}{c}\text { Dec } \\
(\mathrm{J} 2000)\end{array}$ & $z$ & QF & EL \\
\hline MI-21a & & & & \\
SW-70b / & 342.17892 & -44.53668 & 3.6067 & 2 & $\mathrm{~N}$ \\
MI-21b & & & & & \\
NE-97 & 342.19100 & -44.52679 & 3.7131 & 93 & $\mathrm{Y}$ \\
SW-52a/ & 342.17910 & -44.53863 & 4.1130 & 92 & $\mathrm{Y}$ \\
MI-18a & & & & & \\
SW-52b/ & 342.18164 & -44.53936 & 4.1130 & 92 & $\mathrm{Y}$ \\
MI-18b & & & & & \\
NE-98a/ & 342.19015 & -44.53094 & 5.0510 & 92 & $\mathrm{Y}$ \\
MI-98a & & & & & \\
NE-98b/ & 342.19085 & -44.53566 & 5.0510 & 92 & $\mathrm{Y}$ \\
MI-98b & & & & & \\
NE-99a/ & 342.18378 & -44.52122 & 5.2373 & 92 & $\mathrm{Y}$ \\
MI-99a & & & & & \\
NE-99b/ & 342.18874 & -44.52276 & 5.2373 & 92 & $\mathrm{Y}$ \\
MI-99b & & & & & \\
NE-100/ & 342.19701 & -44.52212 & 5.894 & 91 & $\mathrm{Y}$ \\
MI-100b & & & & & \\
SW-53a/ & 342.18104 & -44.53460 & 6.1074 & 92 & $\mathrm{Y}$ \\
MI-14b & & & & & \\
SW-53b/ & 342.19089 & -44.53746 & 6.1074 & 92 & $\mathrm{Y}$ \\
MI-14a & & & & & \\
NE-101c & 342.18402 & -44.53159 & 6.1074 & 4 & $\mathrm{Y}$ \\
MI-14e & & & & & \\
NE-101d/ & 342.18910 & -44.53001 & 6.1074 & 4 & $\mathrm{Y}$ \\
MI-14c & & & & & \\
SW-54 & 342.18407 & -44.53532 & 6.1074 & 91 & $\mathrm{Y}$ \\
\hline
\end{tabular}

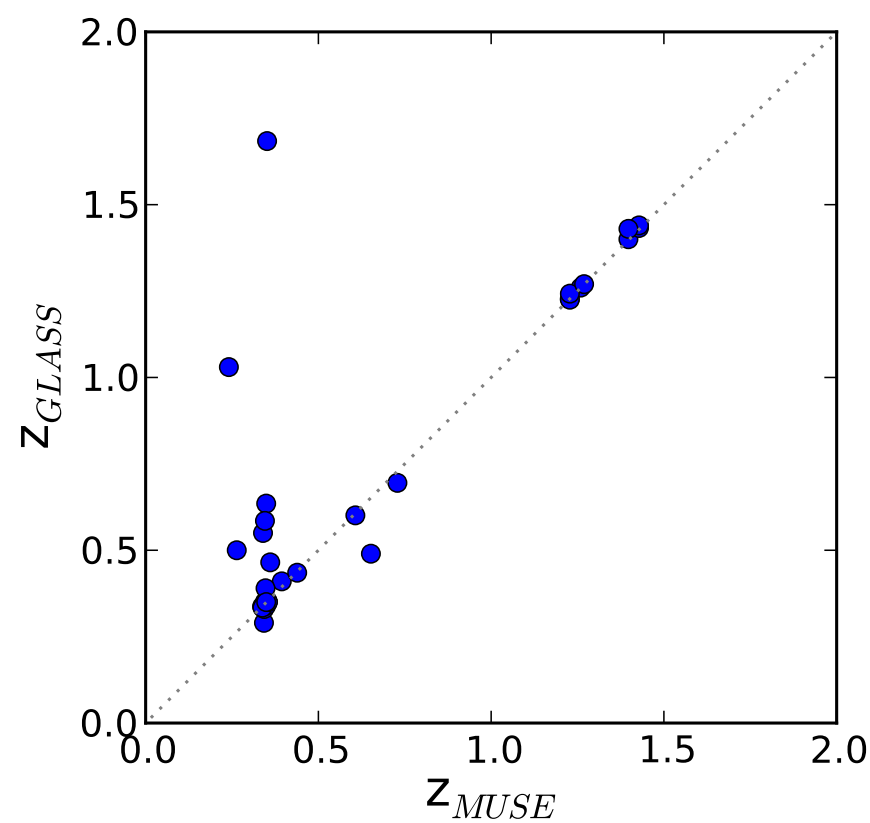

Fig. A.1. Comparison of spectroscopic redshifts from this study and GLASS (Treu et al. 2015; Schmidt et al. 2016). 


\section{Appendix B: Lensing models}

For an individual background galaxy which is multiply imaged, the positions of all images are determined by the relative distance of lens and object, and the mass distribution in the lens. A larger number of multiple image families with spectroscopic redshifts therefore allows one to more accurately model the mass distribution of the cluster. We updated our previous strong lensing model ID F1-5th, as presented in C16, by using the new redshift information. In the updated model we included the extra constraints from 6 additional background sources at different redshifts (sources NE-87, NE-93, NE-94, NE-98, NE-99, and NE91, see Table B.1), totalling 15 multiple image families over the redshift range from $z=0.72$ to $z=6.11$. Here, we describe the model shortly, but we refer the interested reader to $\mathrm{C} 16$ for more details.

We modelled the galaxy cluster using three mass components. The first component is a smooth and extended exponent corresponding to the global dark matter halo, the hot gas present in the cluster, and the intra-cluster light. We model this component by a pseudo-isothermal elliptical mass distribution (hereafter PIEMD, Kassiola \& Kovner 1993). The PIEMD parametrization has six free parameters: the centre position (2), the ellipticity, the orientation, a fiducial velocity dispersion, and the core radius.

The second and third mass components correspond to the BCG and the other galaxy cluster members. The cluster galaxies are modelled using a dual pseudo-isothermal mass distribution (Elíasdóttir et al. 2007; Suyu et al. 2010) with zero ellipticity and core radius and a finite truncation radius. To reduce the number of free parameters, all cluster members except for the BCG have a scaled velocity dispersion and radius, based on their luminosity in the $F 160 \mathrm{~W}$ band. This leaves only 2 free parameters for the full set of cluster members. Because the BCG has a large influence on the central volume of the cluster, we do not scale its velocity dispersion and radius based on its luminosity, but leave these as additional free parameters together with its ellipticity and orientation.

Using these mass components, we model the strong lens using the publicly available software lenstool (Kneib et al. 1996; Jullo et al. 2007). This software minimises the distance between the observed and predicted positions of multiple images based on a Bayesian Markov chain Monte Carlo technique, by varying the free parameters given by the mass distributions.

Our best-fitting model recovers the position of the multiple images with a precision of $\lesssim 0.3^{\prime \prime}$, demonstrating that our model provides a good description of the observations. We calculated the magnification factors, $\mu$, using the median magnification from a MCMC with 10000 points. We found values varying from $\mu=3$ to magnifications of $\mu \approx 50$, see Appendix $C$ for an overview of all magnification factors.
Table B.1. Spectroscopically confirmed multiple images in AS1063, see Table A. 1 for quality flags.

\begin{tabular}{|c|c|c|c|}
\hline ID & RA (J2000) & $\operatorname{Dec}(\mathrm{J} 2000)$ & $z$ \\
\hline $2 a$ & 342.19588 & -44.52895 & $1.2278^{a, b}$ \\
\hline $2 b$ & 342.19450 & -44.52698 & $1.2279^{a, b}$ \\
\hline $2 c$ & 342.18642 & -44.52116 & $1.2277^{a, b}$ \\
\hline $2 d$ & 342.19520 & -44.52786 & 1.2279 \\
\hline $3 a$ & 342.19271 & -44.53119 & $1.2592^{a, b}$ \\
\hline $3 b$ & 342.19213 & -44.52983 & $1.2593^{a, b}$ \\
\hline $3 c$ & 342.17983 & -44.52156 & - \\
\hline $4 a$ & 342.19317 & -44.53653 & 1.3972 \\
\hline $4 \mathrm{~b}$ & 342.18783 & -44.52731 & $1.3972^{a, b}$ \\
\hline $4 \mathrm{c}$ & 342.17921 & -44.52359 & $1.3972^{a, b}$ \\
\hline $6 a$ & 342.18847 & -44.53998 & $1.428^{a, b, c}$ \\
\hline $6 b$ & 342.17585 & -44.53254 & $1.428^{c}$ \\
\hline $6 c$ & 342.17420 & -44.52831 & $1.428^{c, d}$ \\
\hline $7 \mathrm{a}$ & 342.18006 & -44.53842 & $1.035^{c}$ \\
\hline $7 \mathrm{~b}$ & 342.17554 & -44.53590 & $1.035^{c}$ \\
\hline $7 \mathrm{c}$ & 342.17191 & -44.53023 & $1.035^{c}$ \\
\hline $8 a$ & 342.18186 & -44.54050 & $1.837^{a}$ \\
\hline $8 b$ & 342.17424 & -44.53711 & $1.837^{a}$ \\
\hline $8 \mathrm{c}$ & 342.16938 & -44.52726 & - \\
\hline $11 \mathrm{a}$ & 342.17505 & -44.54102 & $3.116^{c, e}$ \\
\hline $11 \mathrm{~b}$ & 342.17315 & -44.53999 & $3.116^{a, b, c, e, f}$ \\
\hline $11 \mathrm{c}$ & 342.16557 & -44.52953 & - \\
\hline $13 a$ & 342.19369 & -44.53014 & 1.2583 \\
\hline $13 b$ & 342.19331 & -44.52942 & 1.2583 \\
\hline $14 \mathrm{a}$ & 342.19088 & -44.53747 & $6.107^{b, c, g}$ \\
\hline $14 \mathrm{~b}$ & 342.18106 & -44.53462 & $6.107^{b, c, g}$ \\
\hline $14 \mathrm{c}$ & 342.18904 & -44.53004 & 6.107 \\
\hline $14 d$ & 342.17129 & -44.51982 & $6.107^{b, g}$ \\
\hline $14 \mathrm{e}$ & 342.18402 & -44.53159 & 6.107 \\
\hline $18 \mathrm{a}$ & 342.18150 & -44.53936 & $4.113^{c}$ \\
\hline $18 \mathrm{~b}$ & 342.17918 & -44.53870 & $4.113^{c}$ \\
\hline $20 \mathrm{a}$ & 342.18745 & -44.53869 & $3.118^{a, h}$ \\
\hline $20 b$ & 342.17886 & -44.53587 & $3.118^{a, h}$ \\
\hline $20 \mathrm{c}$ & 342.17065 & -44.52209 & - \\
\hline $21 \mathrm{a}$ & 342.18586 & -44.53883 & 3.606 \\
\hline $21 b$ & 342.17892 & -44.53668 & 3.606 \\
\hline $87 \mathrm{a}$ & 342.18429 & -44.52529 & 0.7287 \\
\hline $87 \mathrm{~b}$ & 342.18894 & -44.52864 & 0.7287 \\
\hline $87 \mathrm{c}$ & 342.19010 & -44.53010 & 0.7287 \\
\hline $91 \mathrm{a}$ & 342.19238 & -44.52505 & 2.9760 \\
\hline $91 \mathrm{~b}$ & 342.18151 & -44.52025 & - \\
\hline $91 \mathrm{c}$ & 342.19838 & -44.53575 & - \\
\hline $93 a$ & 342.18283 & -44.52028 & 3.169 \\
\hline $93 b$ & 342.19196 & -44.52409 & 3.169 \\
\hline $94 a$ & 342.18935 & -44.51871 & 3.2857 \\
\hline $94 b$ & 342.19615 & -44.52291 & 3.2857 \\
\hline $98 \mathrm{a}$ & 342.19015 & -44.53093 & 5.0510 \\
\hline $98 b$ & 342.19085 & -44.53566 & 5.0510 \\
\hline $99 a$ & 342.18378 & -44.52122 & 5.2373 \\
\hline $99 b$ & 342.18874 & -44.52276 & 5.2373 \\
\hline $100 \mathrm{a}$ & 342.19701 & -44.522121 & 5.8940 \\
\hline $100 \mathrm{~b}$ & 342.19010 & -44.517886 & - \\
\hline
\end{tabular}

Notes. Previous redshift determinations by: ${ }^{(a)} \mathrm{C} 16,{ }^{(b)}$ Balestra et al. (2013), ${ }^{(c)}$ Paper I, ${ }^{(d)}$ Richard et al. (2014), ${ }^{(e)}$ Vanzella et al. (2016a), (f) Johnson et al. (2014), ${ }^{(g)}$ Boone et al. (2013), and ${ }^{(h)}$ Caminha et al. (2016a). 


\section{Appendix C: Individual SED fitting results}

In Sect. 4, we showed and discussed the properties of the LAEs after combining the individual results. Here, we present the individual SED-modelling results of all LAEs and the LBG, as shown in Table C. 1 and in Fig. C.1. In addition, we present the magnification factors $\mu$ of all of the LAEs in Table C.1.

Using Kron-radii on different detection images can lead to colour differences when the PSF is not corrected for. As the PSF of HST is relatively small, circular apertures of $2^{\prime \prime}$ to $3^{\prime \prime}$ should not suffer from this effect We have checked this by comparing the results of the SED-fitting obatined using different choices of apertures, and the results are shown in Fig. C.2. Despite some scatter, most of the results are consistent within $1 \sigma$. We note that the estimates of ages show the largest scatter. However, the uncertainties are large on this parameter and the derived ages are still consistently young ( $<100 \mathrm{Myr})$. The one outlier is NE-97, which suffers from a significant increase in errors in the HST photometry compared to the IRAC photometry when using circular $2^{\prime \prime}$ to $3^{\prime \prime}$ apertures. We note however, that this IRAC detection is less reliable than the results suggest, as we failed to visually confirm the presence of this source in the IRAC maps. We therefore deem the results of the fitting using these circular apertures less reliable than the original values obtained using Kron-radii, which was dominated by the HST-photometry. Because the estimates for the stellar properties remain within $1 \sigma$ of the values used in the main body of the paper, we conclude that the results obtained with the Kron-radii are reliable and that are conclusions hold.

We see that the magnification corrected stellar masses of different images are all within $2 \sigma$ of each other, except for SW-52a. The increased difference between these two images is due to the addition of a Hawk-I detection for SW-52b. This extra datapoint constrains the mass of this LAE to a significantly higher value. We find a similar result for the stellar age, SFR, and SSFR, with only SW-52a showing a discrepant result. We therefore conclude that by taking the average of the different lens-corrected results provides reliable estimates for the stellar parameters, except for SW-52a where we adopt the values obtained from SW-52b.
In the last column of Table C.1, we show the determined escape fraction of Ly $\alpha$. We find large differences between the escape fractions calculated for different images, although these are often within the error estimates. It is peculiar to see that $\mathrm{SW}-52 \mathrm{~b}$ has $f_{\text {esc }}>1$, indicating that either the Ly $\alpha$ flux is boosted (Laursen et al. 2013; Gronke \& Dijkstra 2014), or that the SFR based on the SED modelling is underestimated. We find the latter more likely given that the SFR $\mathrm{SED}_{\text {of }} \mathrm{SW}-52 \mathrm{a}$ is significantly higher.

Most of the photometric data used in this paper is in the UVrestframe, and as a result it is dominated by young stars of which the mass-to-light ratio can vary significantly. To test the reliability of our SED fitting we performed two tests.

First, we constrained the stellar age of our SEDs to $100 \mathrm{Myr}$. We found that the stellar masses increase when setting the age rather than leaving it as a free parameter, see Fig. C.3. However, we find that the decrease is only $\sim 0.5$ dex for the lowest stellar masses in our sample and decreases with increasing stellar mass. As expected, the SFRs are lower when setting the age to $100 \mathrm{Myr}$ but for most galaxies they agree within $2 \sigma$. The older stellar age have lower sSFRs by construction, and it is therefore not surprising that we find significantly lower values. It is important to note that the $\chi^{2}$ values of galaxies with ages well below $100 \mathrm{Myr}$ and small errors increase significantly.

Second, we used the $K$-band data to probe the maximum effect adding restframe optical data would have. For those galaxies where we have no $K$-band detection, we insterted a $K$-band magnitude equal to the $3 \sigma$ depth in our catalogue. This corresponds to a conservative upperlimit and therefore illustrates the maximum effect an older or redder population might have. We show in Fig. C. 4 that there is very little difference between the results when we include mock $3 \sigma K$-band detections and the original photometry.

These tests show that although we are mostly using UV restframe data, the properties derived are reliable and our results remain intact even using different assumptions. We note that a red and old population might still be present as three datapoints are not able to securely determine this, but it does constrain their maximal contribution. 
ID: NE-93b, $z=3.169$

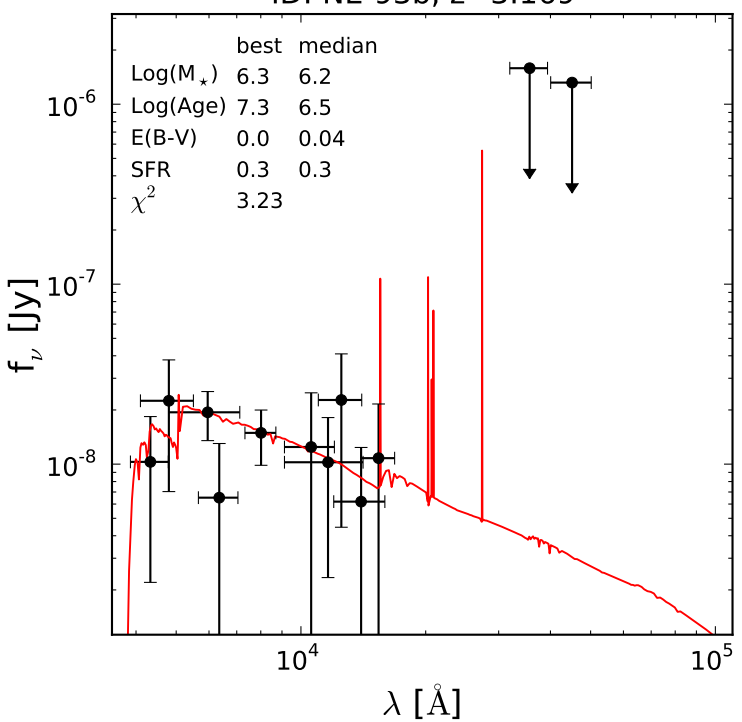

ID: NE-94a, z=3.2857

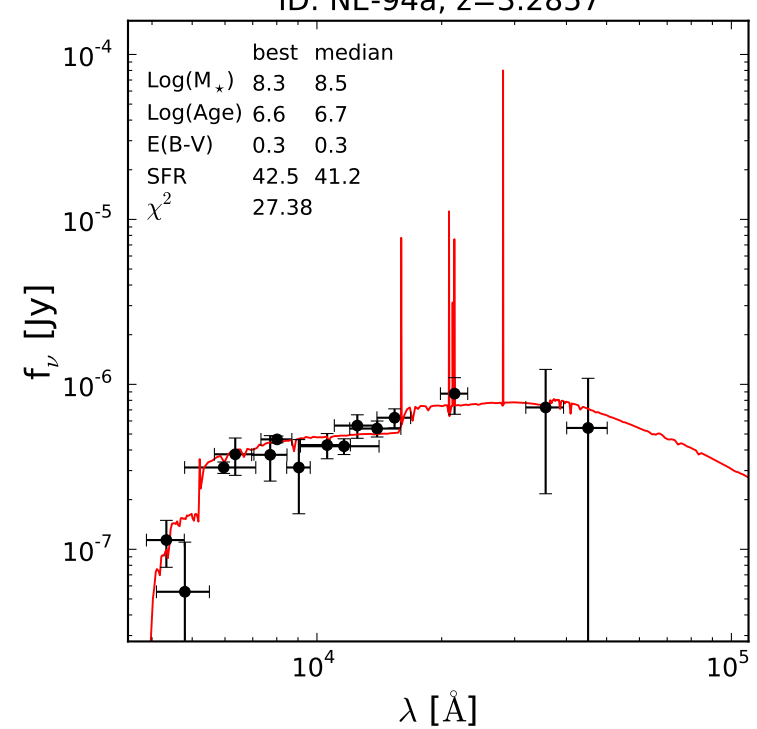

ID: NE-98a, $z=5.051$

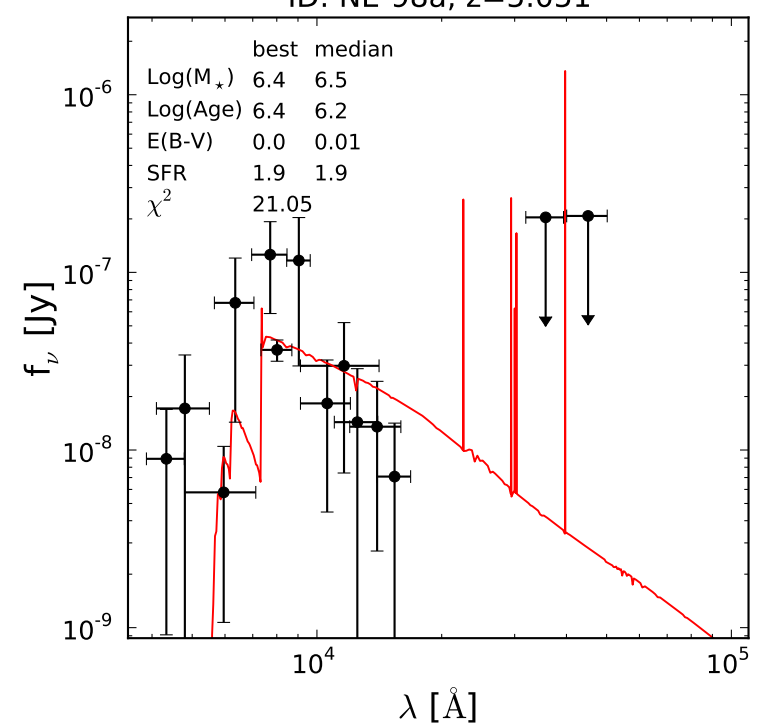

ID: NE-93a, z=3.169

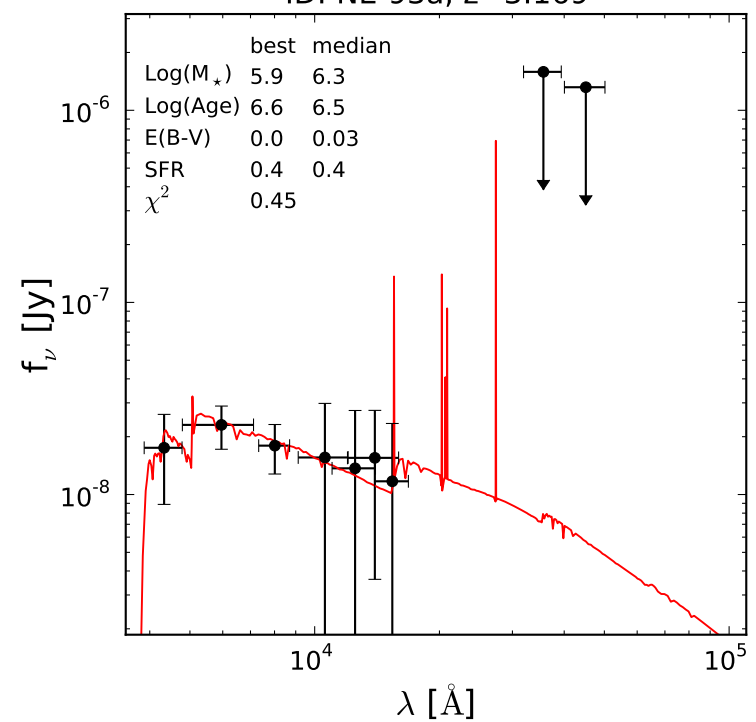

ID: NE-94b, $z=3.2857$

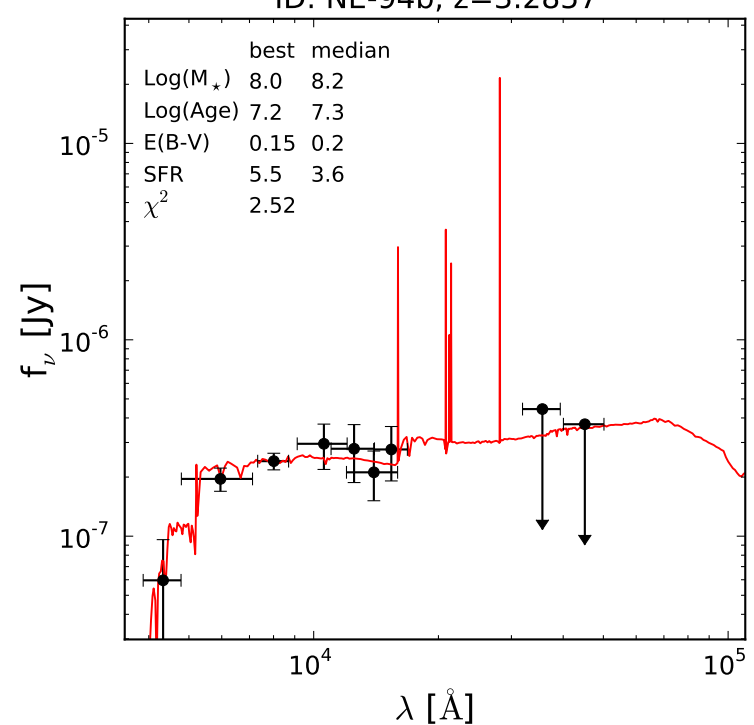

ID: NE-98b, $z=5.051$

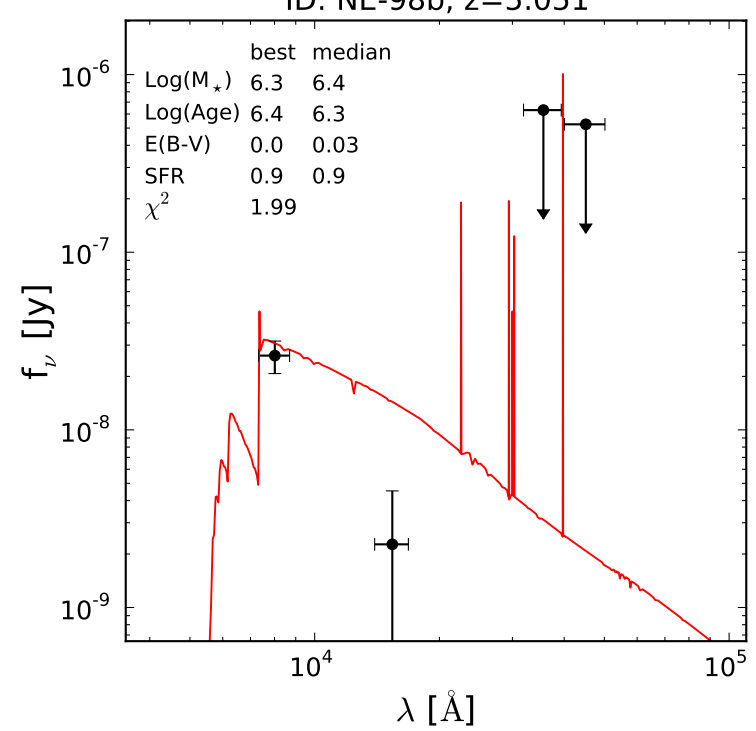

Fig. C.1. Sed fitting results of individual LAEs. The red lines correspond to the best-fitting SED-model and the black circles are the photometric datapoints with $2 \sigma$-errorbars. The details of the best fitting model and the median marginalized values for each galaxy are shown wiht text in the upper left corners. 
W. Karman et al.: Properties of low luminosity LAEs at $z>3$

ID: SW-49a, z=3.116

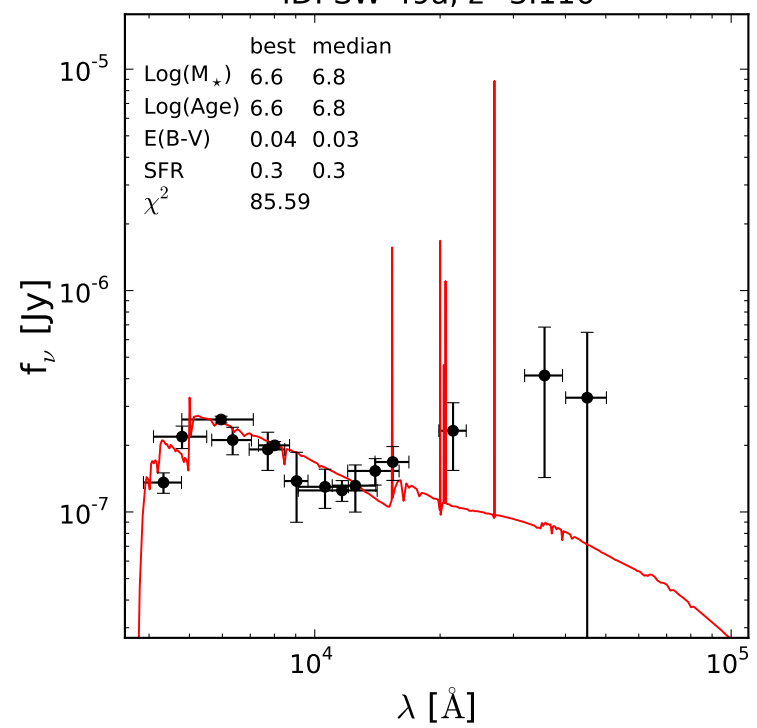

ID: SW-52a, $z=4.113$

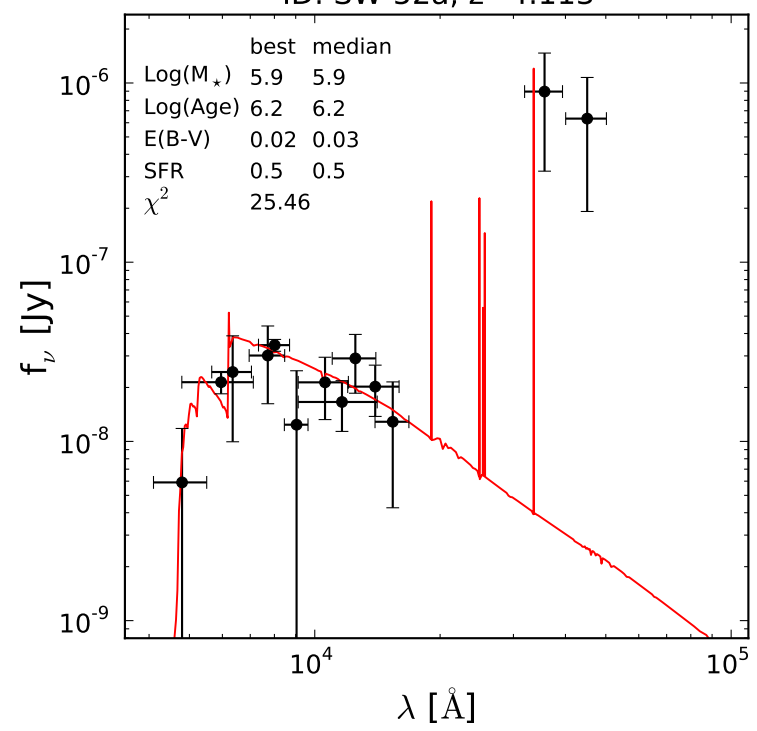

ID: SW-53a, z=6.107

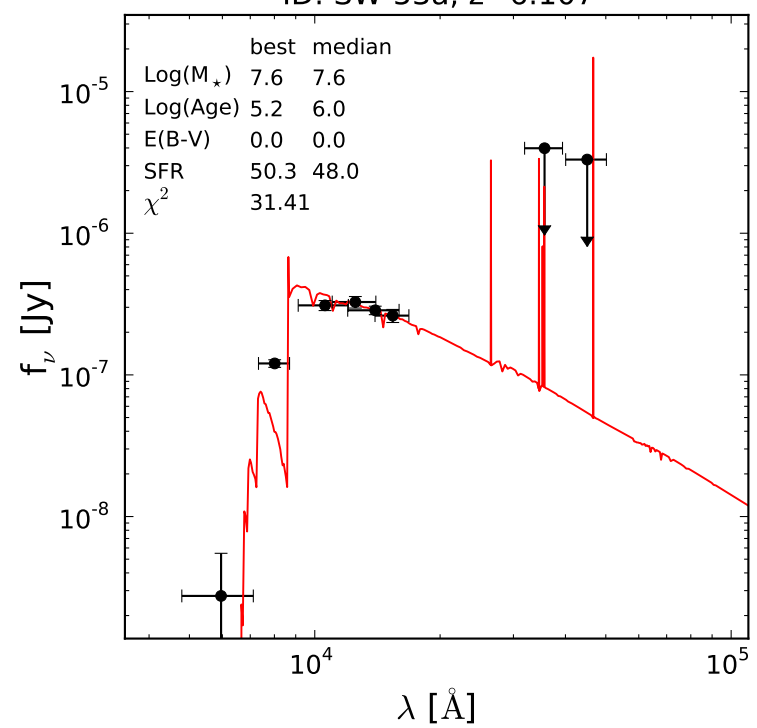

ID: SW-49b, z=3.116

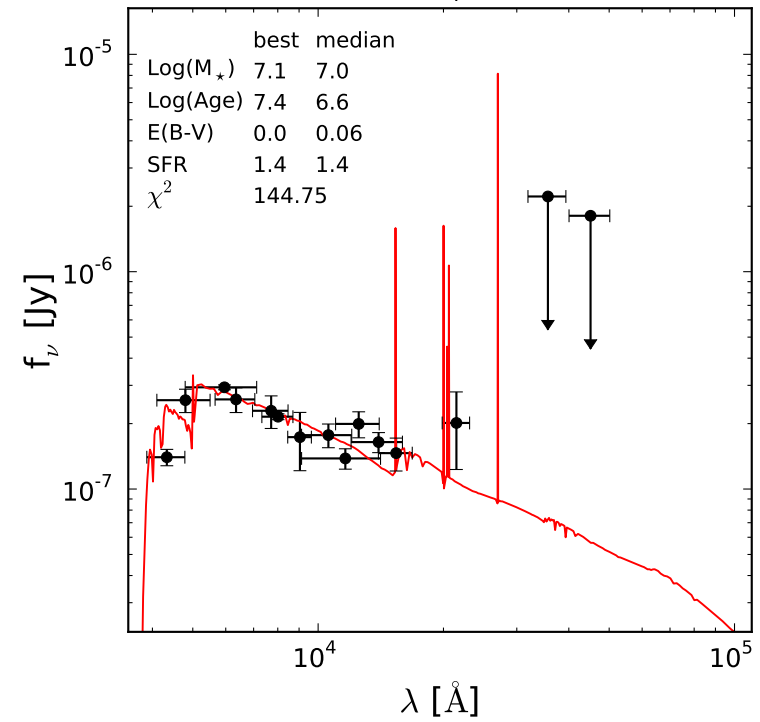

ID: $S W-52 b, z=4.113$

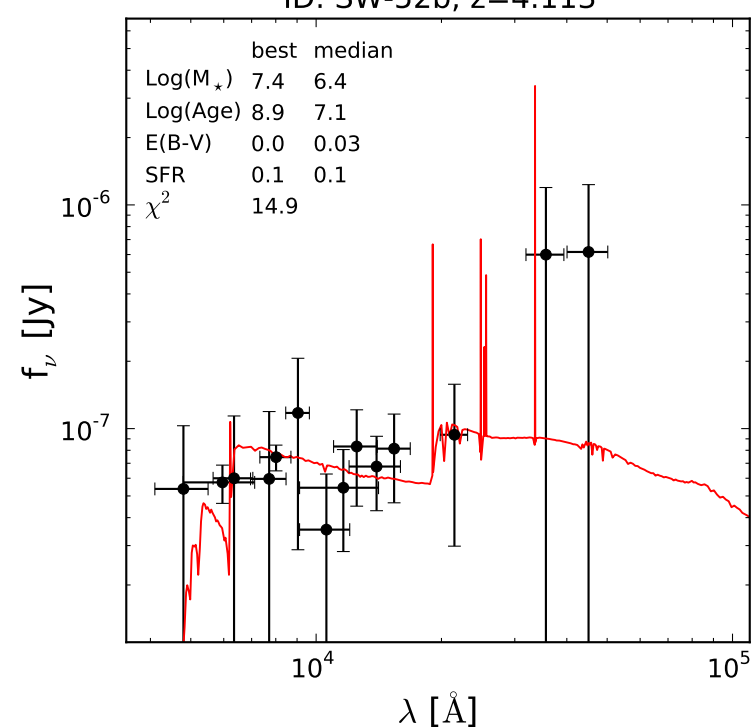

ID: SW-53b, $z=6.107$

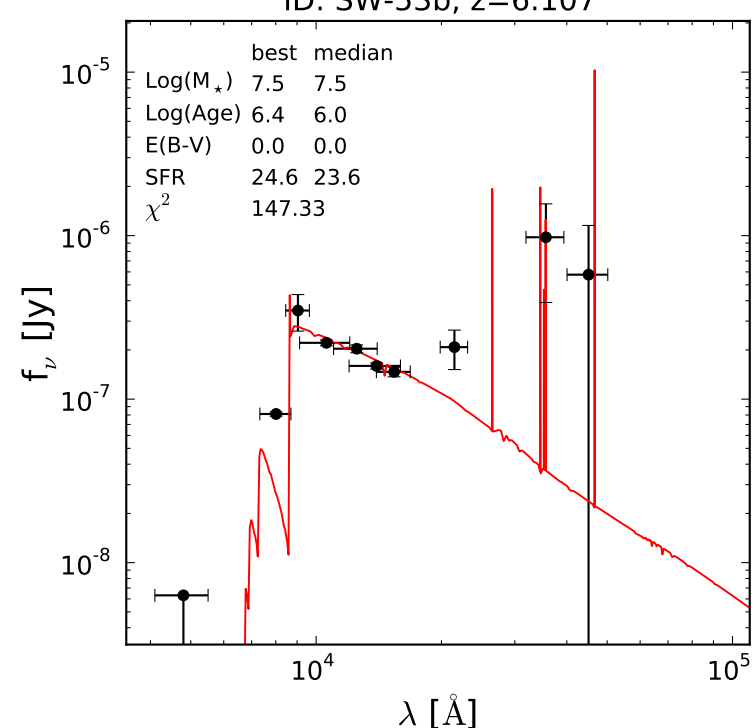

Fig. C.1. continued. 
ID: SW-50, z=3.117

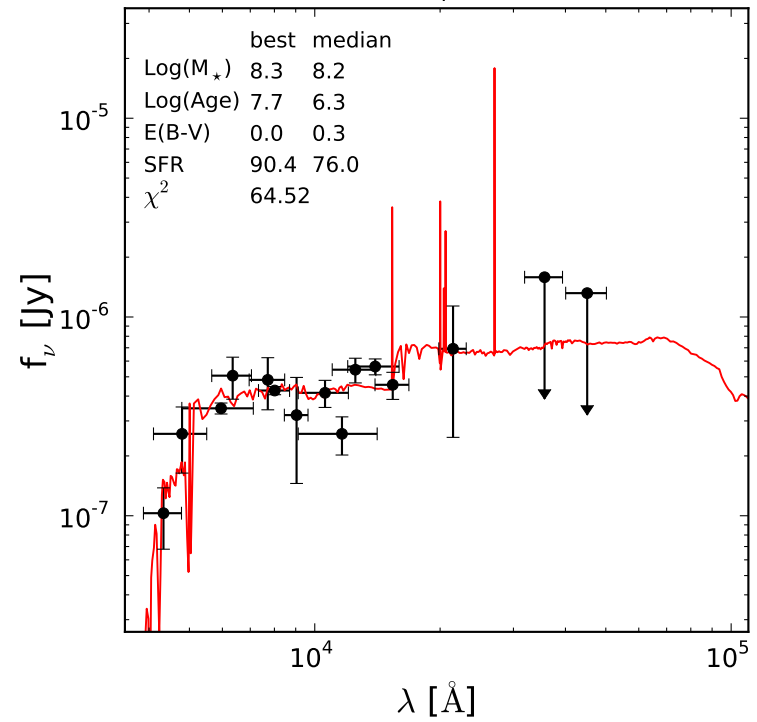

ID: SW-68a, $z=3.117$

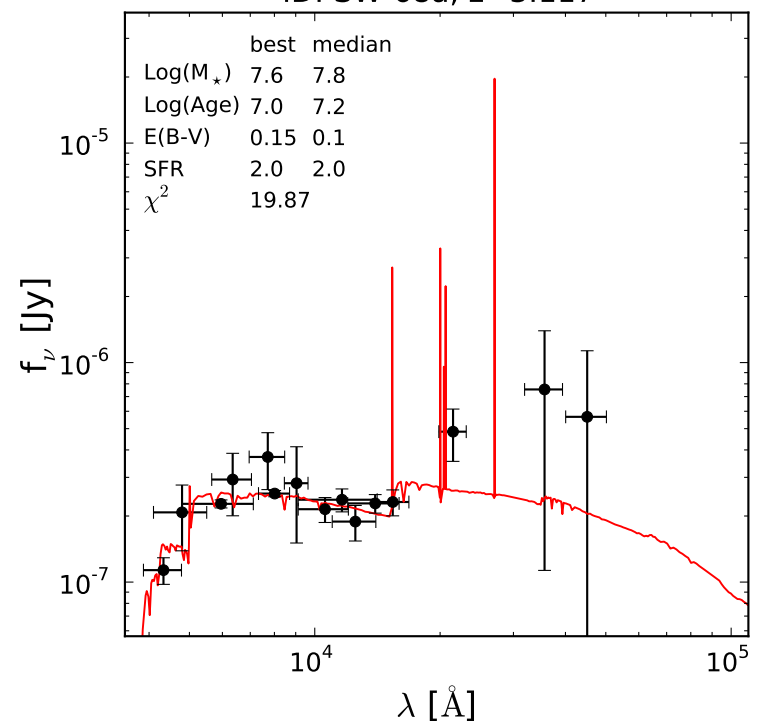

ID: NE-91, z=2.976

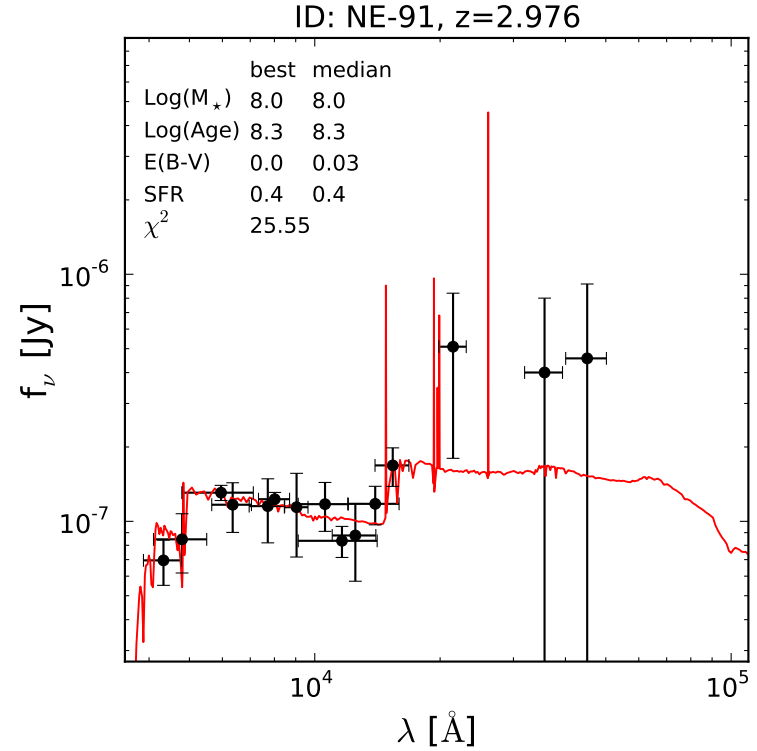

ID: SW-51, $z=3.228$

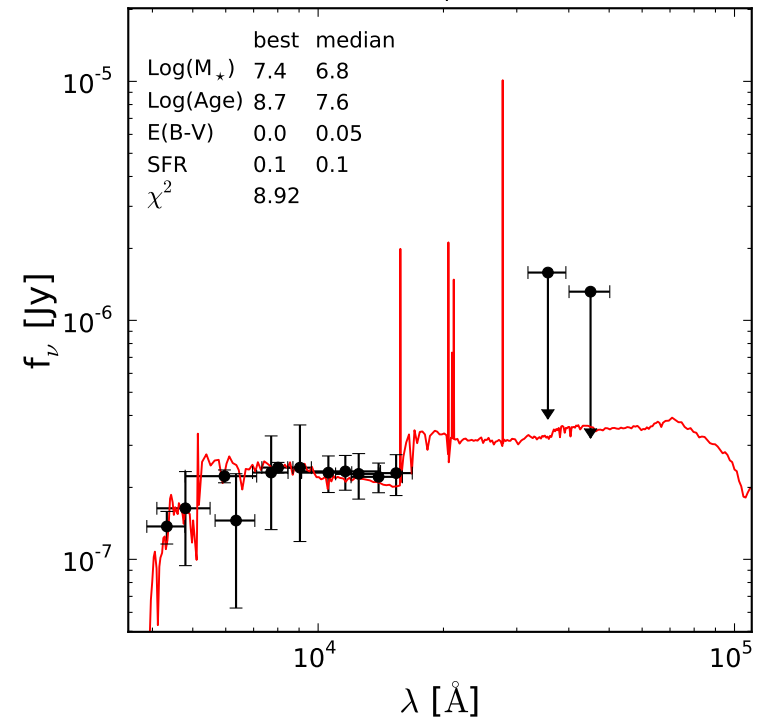

ID: NE-96, z=3.4514

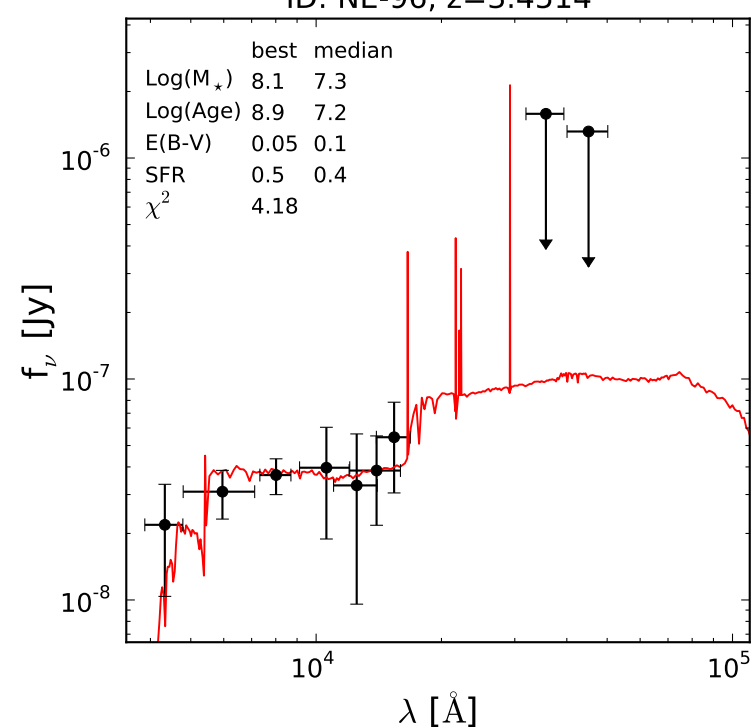

ID: NE-97, z=3.7131

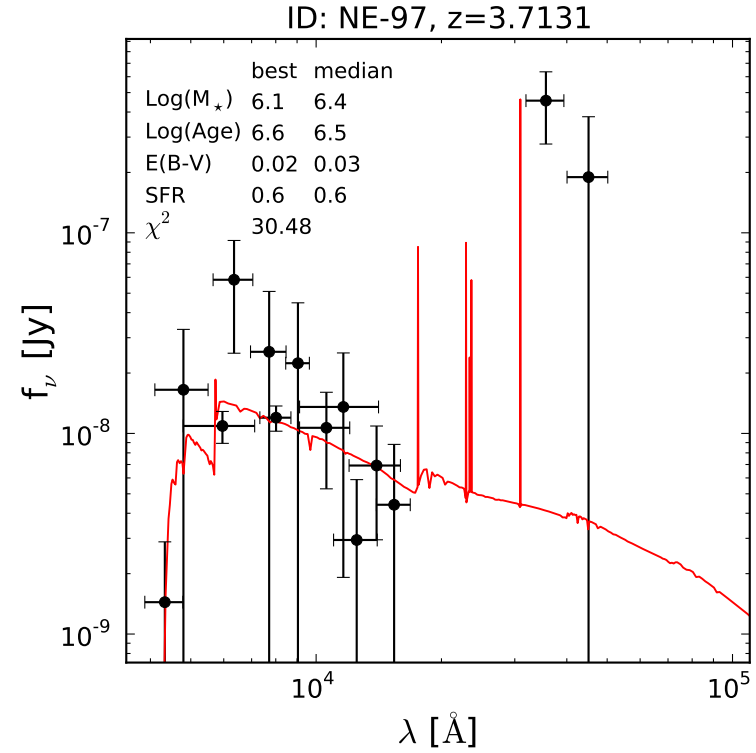

Fig. C.1. continued. 
W. Karman et al.: Properties of low luminosity LAEs at $z>3$
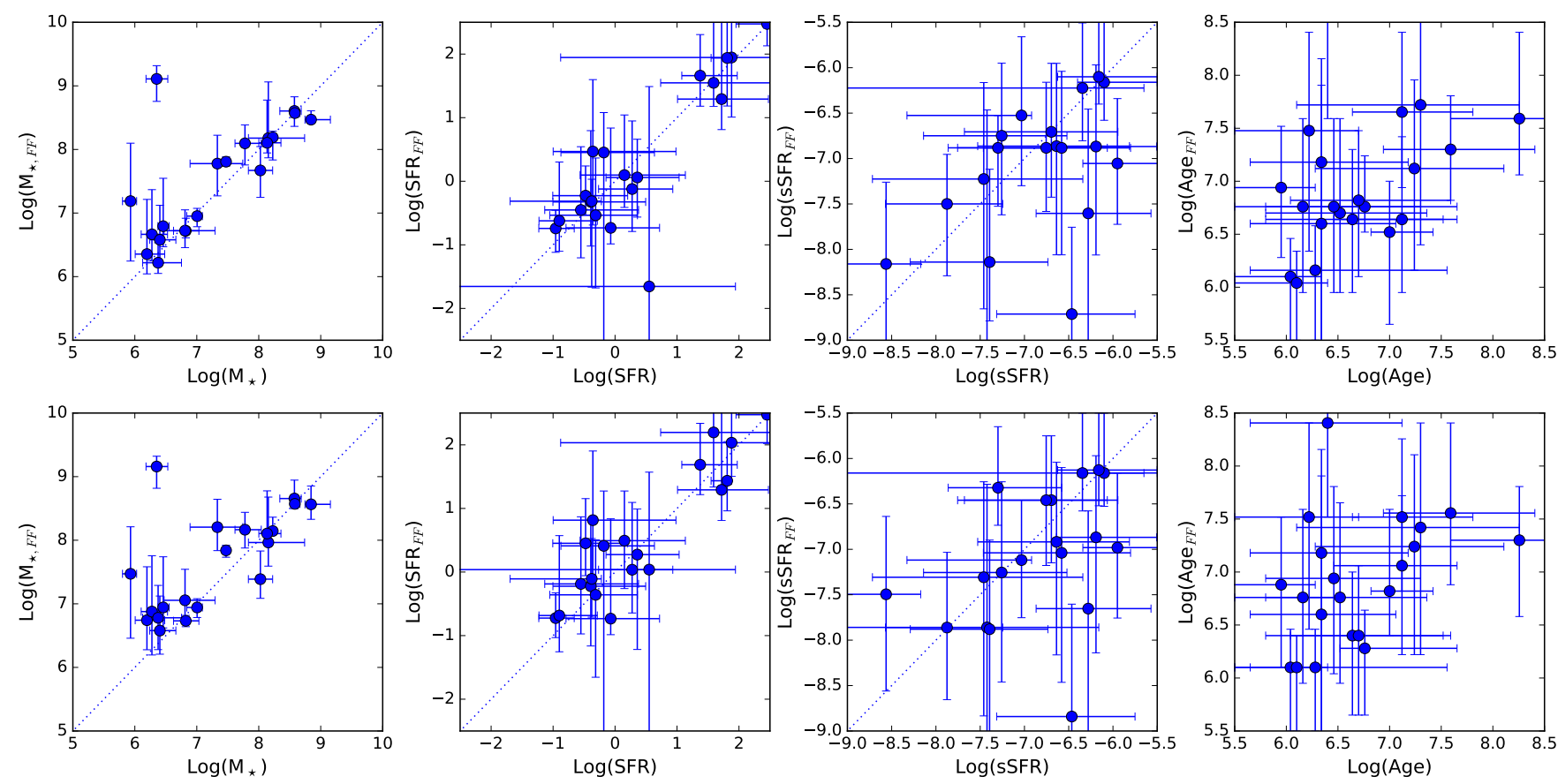

Fig. C.2. Comparison of the SED-fitting results obtained using the Kron-radii photometry using Kron-radii on the $x$-axis versus results obtained using a $2^{\prime \prime}$ aperture (top row) or $3^{\prime \prime}$ (bottom row) on the $y$-axis. The panels show, from left to right, the stellar mass, the SFR, the sSFR, and stellar age.
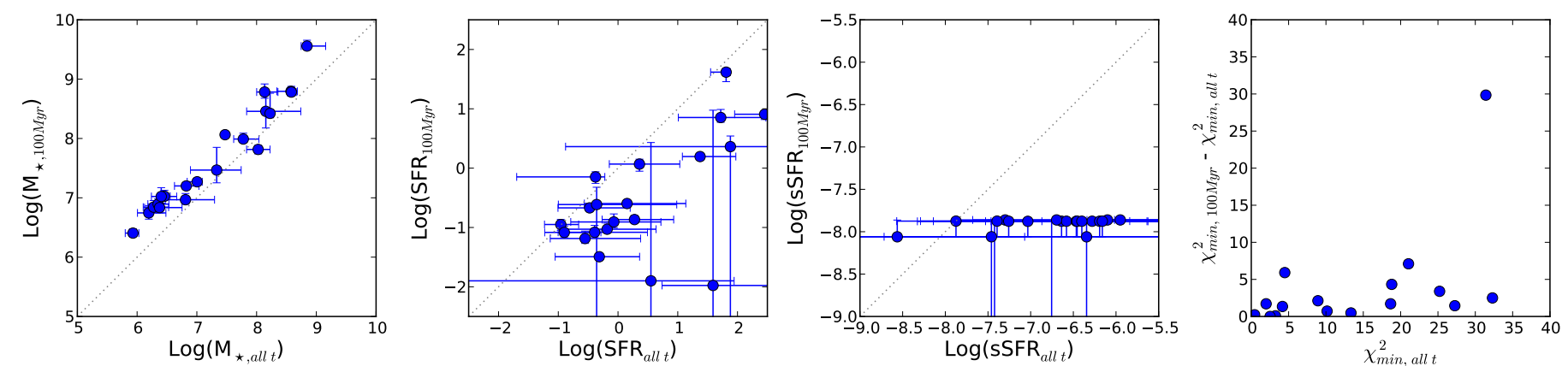

Fig. C.3. Comparison of the SED-fitting results obtained when leaving the age free ( $x$-axis) versus those obtained for a fixed stellar age of $100 \mathrm{Myr}$ ( $y$-axis). The panels show, from left to right, the stellar mass, the SFR, the sSFR, and the difference in $\chi^{2}$.
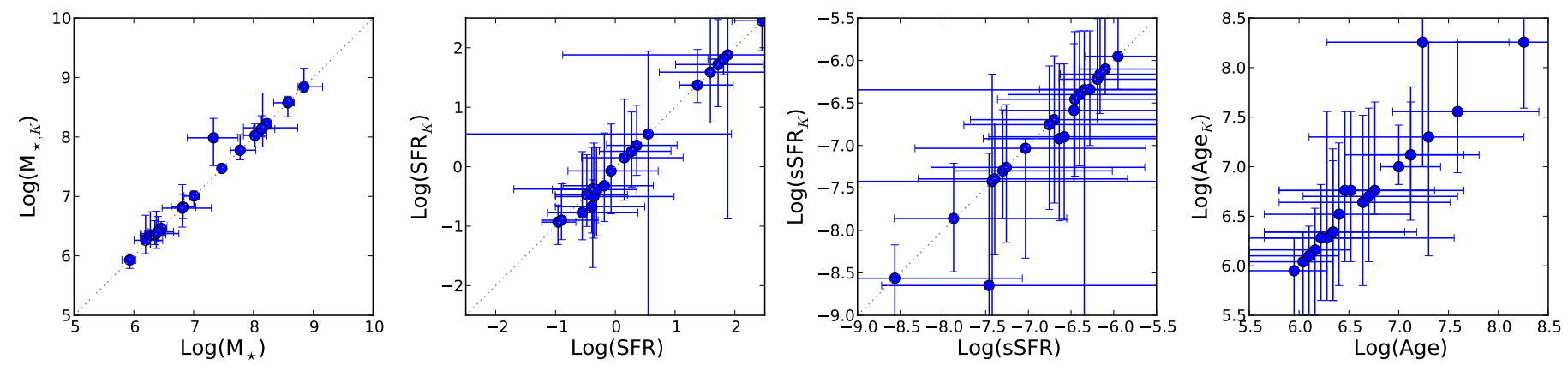

Fig. C.4. Comparison of the SED-fitting results obtained using the original photometry ( $x$-axis) versus those obtained using a catalogue where mock $3 \sigma K$-band detections were inserted for non-detections ( $y$-axis). The panels show, from left to right, the stellar mass, the SFR, the sSFR, and stellar age. 
Table C.1. SED fitting results of every individual LAE image.

\begin{tabular}{|c|c|c|c|c|c|c|c|c|c|}
\hline ID & $\mu$ & $\log \left(M_{\star} / M_{\odot}\right)$ & $\log \left(\mathrm{Age}_{\mathrm{SSP}} / \mathrm{yr}\right)$ & $E(B-V)$ & $\log \left(S F R /\left(M_{\odot} \mathrm{yr}^{-1}\right)\right)$ & $\log (\mathrm{s} S F R / \mathrm{yr})$ & $\beta$ & $f_{\text {esc,Ly } \alpha}$ & $M_{F 814 W}$ \\
\hline NE-91 & $4.41 \pm 0.11$ & $8.04_{-0.20}^{+0.19}$ & $8.26_{-0.67}^{+0.45}$ & $0.03_{-0.02}^{+0.03}$ & $-0.38_{-1.30}^{+0.15}$ & $-8.56_{-0.70}^{+0.39}$ & $-2.42 \pm 0.09$ & 0.18 & 26.176 \\
\hline SW-49a & $16.50 \pm 1.00$ & $6.79_{-0.17}^{+0.27}$ & $6.82_{-0.24}^{+0.83}$ & $0.03_{-0.03}^{+0.03}$ & $-0.52_{-0.52}^{+0.63}$ & $-7.42_{-0.45}^{+0.78}$ & $-2.79 \pm 0.10$ & 2.10 & 25.648 \\
\hline SW-49b & $15.80 \pm 0.92$ & $7.01_{-0.17}^{+0.09}$ & $6.64_{-0.84}^{+0.88}$ & $0.06_{-0.05}^{+0.02}$ & $0.15_{-0.72}^{+0.98}$ & $-6.70_{-0.98}^{+0.76}$ & $-2.79 \pm 0.05$ & 0.68 & 25.568 \\
\hline SW-50 & $11.20 \pm 0.76$ & $8.23_{-0.06}^{+0.05}$ & $6.28_{-0.63}^{+1.28}$ & $0.30_{-0.20}^{+0.00}$ & $1.88_{-2.76}^{+0.69}$ & $-6.34_{-2.12}^{+0.69}$ & $-1.95 \pm 0.05$ & 0.01 & 24.825 \\
\hline SW-68a & $6.09 \pm 0.12$ & $7.78_{-0.15}^{+0.28}$ & $7.18_{-0.42}^{+0.78}$ & $0.10_{-0.05}^{+0.10}$ & $0.30_{-0.52}^{+0.48}$ & $-7.50_{-0.83}^{+0.63}$ & $-2.09 \pm 0.08$ & 0.56 & 25.392 \\
\hline NE-93a & $4.95 \pm 0.12$ & $6.28_{-0.17}^{+0.25}$ & $6.46_{-0.66}^{+0.84}$ & $0.03_{-0.02}^{+0.03}$ & $-0.39_{-0.61}^{+0.89}$ & $-6.58_{-0.88}^{+0.78}$ & $-2.85 \pm 0.50$ & 0.63 & 28.264 \\
\hline NE-93b & $5.08 \pm 0.11$ & $6.20_{-0.19}^{+0.29}$ & $6.52_{-0.72}^{+0.84}$ & $0.04_{-0.03}^{+0.04}$ & $-0.55_{-0.58}^{+0.93}$ & $-6.64_{-0.89}^{+0.82}$ & $-2.85 \pm 0.41$ & 1.23 & 28.466 \\
\hline SW-51 & $55.60 \pm 8.95$ & $6.81_{-0.34}^{+0.49}$ & $7.59_{-0.65}^{+0.82}$ & $0.05_{-0.03}^{+0.05}$ & $-0.96_{-0.27}^{+0.30}$ & $-7.87_{-0.69}^{+0.63}$ & $-2.31 \pm 0.05$ & 0.27 & 25.440 \\
\hline NE-94a & $7.09 \pm 0.15$ & $8.50_{-0.16}^{+0.16}$ & $6.70_{-0.66}^{+0.66}$ & $0.30_{-0.05}^{+0.05}$ & $1.61_{-0.59}^{+0.93}$ & $-6.74_{-0.75}^{+0.64}$ & $-1.76 \pm 0.08$ & 0.03 & 24.734 \\
\hline NE-94b & $8.12 \pm 0.21$ & $8.15_{-0.32}^{+0.59}$ & $7.30_{-1.20}^{+0.96}$ & $0.20_{-0.10}^{+0.10}$ & $0.55_{-0.65}^{+1.39}$ & $-7.42_{-1.64}^{+1.26}$ & $-1.85 \pm 0.18$ & 0.07 & 25.445 \\
\hline NE-96 & $4.78 \pm 0.13$ & $7.33_{-0.44}^{+0.40}$ & $7.24_{-0.96}^{+0.87}$ & $0.10_{-0.07}^{+0.15}$ & $-0.36_{-0.64}^{+1.34}$ & $-7.48_{-1.24}^{+1.14}$ & $-2.14 \pm 0.22$ & 0.52 & 27.487 \\
\hline NE-97 & $2.98 \pm 0.06$ & $6.35_{-0.17}^{+0.20}$ & $6.46_{-0.66}^{+0.72}$ & $0.03_{-0.02}^{+0.03}$ & $-0.23_{-0.66}^{+0.84}$ & $-6.52_{-0.85}^{+0.72}$ & $-2.90 \pm 0.15$ & 0.27 & 28.704 \\
\hline SW-52a & $23.6 \pm 1.47$ & $5.93_{-0.13}^{+0.10}$ & $6.22_{-0.72}^{+0.48}$ & $0.03_{-0.02}^{+0.02}$ & $-0.32_{-0.74}^{+0.68}$ & $-6.28_{-0.60}^{+0.71}$ & $-3.05 \pm 0.10$ & 0.32 & 27.557 \\
\hline SW-52b & $27.9 \pm 1.56$ & $6.40_{-0.26}^{+0.39}$ & $7.12_{-0.60}^{+0.60}$ & $0.03_{-0.02}^{+0.03}$ & $-0.91_{-0.33}^{+0.56}$ & $-7.30_{-0.86}^{+0.73}$ & $-2.50 \pm 0.28$ & 2.46 & 26.719 \\
\hline NE-98a & $8.84 \pm 0.35$ & $6.46_{-0.09}^{+0.10}$ & $6.16_{-0.66}^{+0.36}$ & $0.01_{-0.01}^{+0.02}$ & $0.28_{-0.54}^{+0.66}$ & $-6.19_{-0.45}^{+0.68}$ & $-3.17 \pm 0.02$ & 0.24 & 27.491 \\
\hline NE-98b & $8.95 \pm 0.24$ & - & - & - & - & - & - & - & 27.855 \\
\hline NE-99a & $9.91 \pm 0.33$ & - & - & - & - & - & - & - & 28.854 \\
\hline NE-99b & $10.10 \pm 0.29$ & - & - & - & - & - & - & - & 29.584 \\
\hline NE-100 & $7.87 \pm 0.21$ & - & - & - & - & - & - & - & - \\
\hline NE-101d & $6.53 \pm 0.51$ & $8.13_{-0.13}^{+0.23}$ & $6.34_{-0.69}^{+0.72}$ & $0.02_{-0.02}^{+0.03}$ & $1.72_{-0.71}^{+0.76}$ & $-6.40_{-0.84}^{+0.75}$ & $-3.18 \pm 0.00$ & 0.17 & - \\
\hline SW-53a & $5.56 \pm 0.33$ & $7.59_{-0.02}^{+0.08}$ & $5.95_{-0.60}^{+0.33}$ & $0.00_{-0.00}^{+0.01}$ & $1.68_{-0.41}^{+0.56}$ & $-5.95_{-0.39}^{+0.60}$ & $-3.02 \pm 0.05$ & 0.09 & 26.198 \\
\hline SW-53b & $5.97 \pm 0.12$ & $7.47_{-0.00}^{+0.00}$ & $6.04_{-0.54}^{+0.30}$ & $0.00_{-0.00}^{+0.00}$ & $1.37_{-0.30}^{+0.60}$ & $-6.10_{-0.30}^{+0.60}$ & $-3.17 \pm 0.02$ & 0.43 & 26.627 \\
\hline SW-70a ${ }^{a}$ & $8.43 \pm 0.248$ & $8.58_{-0.01}^{+0.08}$ & $6.10_{-0.60}^{+0.30}$ & $0.25_{-0.00}^{+0.00}$ & $2.45_{-0.50}^{+0.63}$ & $-6.16_{-0.43}^{+0.66}$ & $-2.00 \pm 0.02$ & 0.00 & 23.233 \\
\hline $\mathrm{SW}-70 \mathrm{~b}^{a}$ & $5.79 \pm 0.146$ & $8.84_{-0.10}^{+0.31}$ & $7.00_{-0.18}^{+0.42}$ & $0.20_{-0.00}^{+0.00}$ & $1.81_{-0.26}^{+0.07}$ & $-7.03_{-1.29}^{+0.11}$ & $-1.83 \pm 0.12$ & 0.01 & 24.018 \\
\hline
\end{tabular}

Notes. Results of SED modelling of all individual images of LAEs. The stellar masses and SFRs have been corrected for lensing, using the magnification factor given in the second column. The last column shows the observed magnitude in the $F 814 W$ HST band without correcting for magnification. LAEs NE-98b, NE-99a, NE-99b, and NE-100 did not have enough photometric detections to perform a sensible modelling. ${ }^{(a)}$ Lyman break galaxy, no significant Ly $\alpha$ emission. 


\section{Appendix D: Individual Lyman $\alpha$ modelling results}

We modelled the profile of the Ly $\alpha$ for each LAE with a clear asymmetric profile or $S / N>8$ of the Ly $\alpha$ flux individually ${ }^{13}$, and we show the results in Figs. D.1 to D.18. We used our spectra to constrain the intrinsic redshift based on UV emission lines whenever possible, and we measured the FWHM due to the instrument and weather conditions at every wavelength. We note that there is no clear peak in the FWHM distribution after fitting, and we can therefore not better constrain the values of the FWHM over the narrow, but limited allowed range. However, because there does not seem to be correlation with any of the other parameters, this does not have any implications on the results in this paper. Although the majority of Ly $\alpha$ models are well fit by the models, it is clear that in some spectra, for example NE-98b, NE-99a, and NE-99b, the sky interference forces the models to an incorrect expansion velocity. We have masked out the LAEs where this was a problem in the main body of the paper.

Table D.1. Results from the Ly $\alpha$ line modelling simulations of all images of LAEs.

\begin{tabular}{|c|c|c|c|c|c|c|}
\hline ID & $z$ & $\begin{array}{l}f_{\mathrm{Ly}} \alpha \\
10^{-20} \mathrm{erg} / \mathrm{s} / \mathrm{cm}^{-2}\end{array}$ & $\log \left(L_{\mathrm{Ly}} \alpha /(\mathrm{erg} / \mathrm{s})\right)$ & $\log \left(N_{\mathrm{H}} / \mathrm{cm}^{-2}\right)$ & $\begin{array}{l}v_{\exp } \\
\mathrm{km} \mathrm{s}^{-1}\end{array}$ & $\begin{array}{l}S F R_{\mathrm{Ly} \alpha} \\
M_{\odot} \mathrm{yr}^{-1}\end{array}$ \\
\hline NE-91 & 2.9760 & $4.7 \pm 0.6$ & $40.9 \pm 0.0611$ & - & - & 0.0741 \\
\hline SW-49a & 3.1169 & $179.0 \pm 21.3$ & $42.0 \pm 0.0551$ & $17.1_{0.1}^{+0.2}$ & $72.9_{-3.5}^{+1.6}$ & 0.83 \\
\hline SW-49b & 3.1169 & $198.0 \pm 33.1$ & $42.0 \pm 0.0795$ & $17_{0.1}^{+0.2}$ & $60.7_{-3.7}^{+3.1}$ & 0.965 \\
\hline SW-50 & 3.1169 & $110.0 \pm 2.0$ & $41.9 \pm 0.00803$ & $17.5_{0.2}^{+0.3}$ & $124_{-6}^{+6}$ & 0.757 \\
\hline SW-68a & 3.1166 & $118.0 \pm 1.0$ & $42.2 \pm 0.00364$ & $20_{0.2}^{+0.2}$ & $463_{-48}^{+21}$ & 1.49 \\
\hline NE-93a & 3.1696 & $15.8 \pm 1.6$ & $41.4 \pm 0.0469$ & $19_{0.5}^{+0.4}$ & $42.9_{-16.1}^{+25.3}$ & 0.255 \\
\hline NE-93b & 3.1701 & $21.9 \pm 1.3$ & $41.6 \pm 0.0265$ & $17.7_{0.6}^{+0.9}$ & $46.4_{-11.3}^{+11.6}$ & 0.345 \\
\hline SW-51 & 3.2271 & $19.8 \pm 0.9$ & $40.5 \pm 0.0206$ & $18.5_{0.7}^{+0.5}$ & $98_{-17.8}^{+15}$ & 0.0297 \\
\hline NE-94a & 3.2848 & $37.2 \pm 1.9$ & $41.7 \pm 0.0228$ & $19.4_{0.3}^{+0.3}$ & $192_{-25}^{+19}$ & 0.458 \\
\hline NE-94b & 3.2854 & $44.4 \pm 2.2$ & $41.7 \pm 0.0219$ & $19_{0.2}^{+0.2}$ & $174_{-9}^{+11}$ & 0.477 \\
\hline NE-96 & 3.4514 & $10.9 \pm 0.7$ & $41.4 \pm 0.0291$ & $18.6_{0.3}^{+0.1}$ & $56.7_{-8.9}^{+7.3}$ & 0.224 \\
\hline NE-97 & 3.7131 & $4.6 \pm 0.6$ & $41.3 \pm 0.0581$ & $20.4_{0.2}^{+0.1}$ & $181_{-12}^{+13}$ & 0.181 \\
\hline SW-52a & 4.1130 & $24.9 \pm 0.9$ & $41.2 \pm 0.0165$ & $17.3_{0.2}^{+0.2}$ & $87.8_{-7.9}^{+6}$ & 0.157 \\
\hline SW-52b & 4.1128 & $29.8 \pm 1.1$ & $41.2 \pm 0.0167$ & $17_{0.1}^{+0.1}$ & $108_{-2}^{+5}$ & 0.159 \\
\hline NE-98a & 5.0514 & $16.5 \pm 0.7$ & $41.7 \pm 0.0198$ & $19.4_{0.2}^{+0.2}$ & $149_{-12}^{+12}$ & 0.451 \\
\hline NE-98b & 5.0549 & $4.7 \pm 0.7$ & $41.1 \pm 0.0693$ & $18.2_{0.4}^{+0.4}$ & $-4.93_{-3.36}^{+2.64}$ & 0.127 \\
\hline NE-99a & 5.2399 & $10.5 \pm 1.2$ & $41.5 \pm 0.0506$ & $18.5_{0.6}^{+0.8}$ & $236_{-107}^{+183}$ & 0.28 \\
\hline NE-99b & 5.2415 & $12.1 \pm 1.1$ & $41.5 \pm 0.0423$ & $17.4_{0.3}^{+0.4}$ & $-19.8_{-3.8}^{+5.1}$ & 0.315 \\
\hline NE-100 & 5.8940 & $4.7 \pm 2.5$ & $41.4 \pm 0.329$ & - & - & 0.207 \\
\hline NE-101d & 6.1074 & $150.0 \pm 1.9$ & $43 \pm 0.00546$ & - & - & 8.67 \\
\hline SW-53a & 6.1074 & $63.4 \pm 2.7$ & $42.7 \pm 0.0189$ & $19.8_{-0.1}^{+0.1}$ & $151_{5}^{+3}$ & 4.31 \\
\hline SW-53b & 6.1074 & $161.0 \pm 4.0$ & $43 \pm 0.0109$ & - & - & 10.2 \\
\hline SW-70a & 3.6065 & - & $41.2 \pm 2.0$ & - & - & 0.129 \\
\hline SW-70b & 3.6065 & - & $41.3 \pm 2.0$ & - & - & 0.188 \\
\hline
\end{tabular}

Notes. We report in Col. 2 the intrinsic redshift obtained from modelling the Ly $\alpha$ profile. Column 3 gives the observed Ly $\alpha$ flux without correcting for lensing, while we corrected $L_{\mathrm{Lya}}$ and $\mathrm{SFR}_{\mathrm{Ly} \alpha}$ for the effect of gravitational magnification using the magnification factors given in Table C.1. 

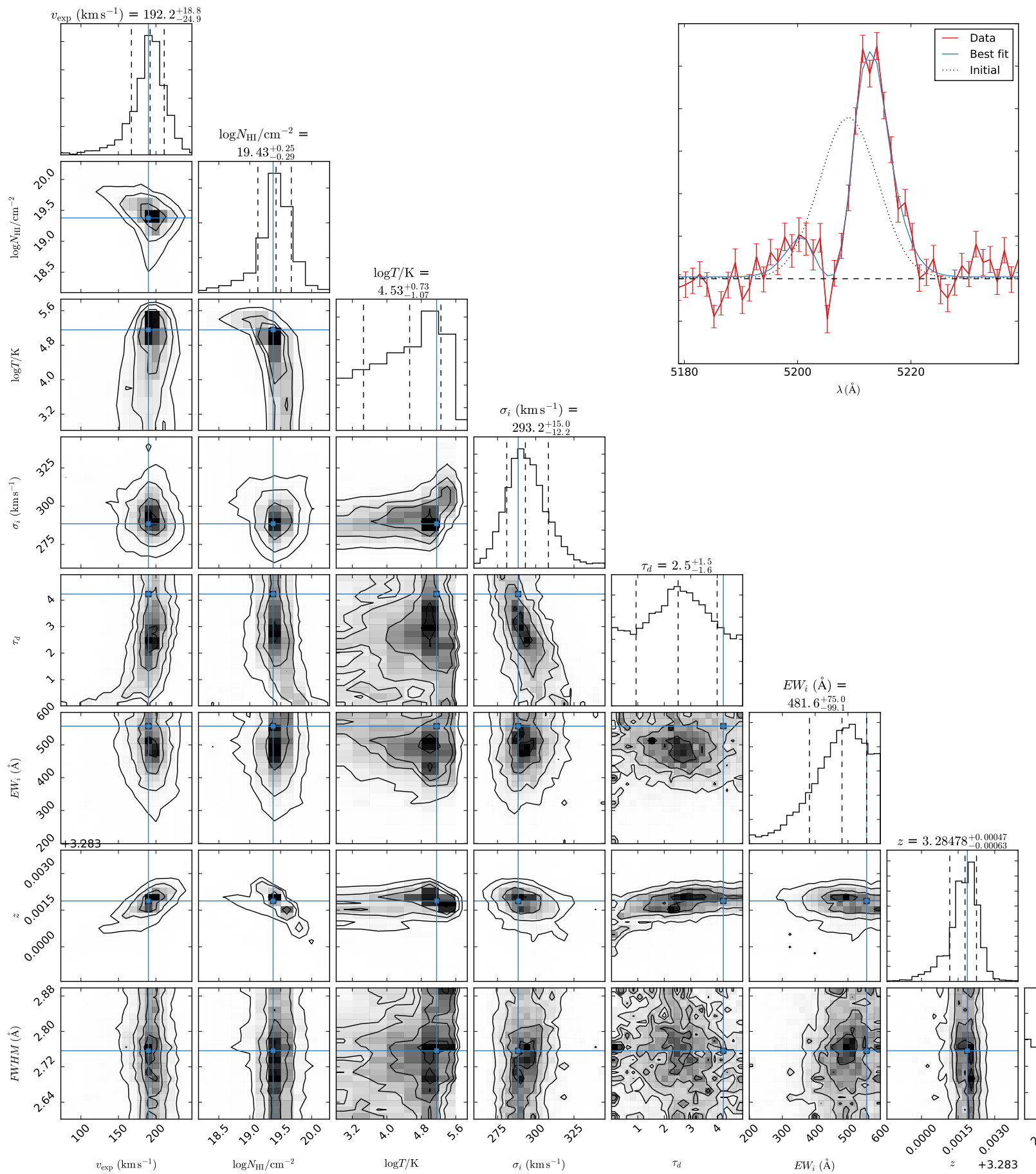

$F W H M(\AA)=$

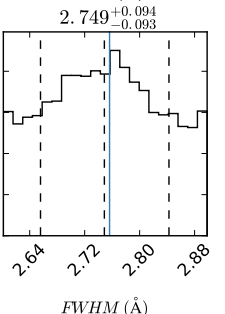

Fig. D.1. Lya modelling results for LAE NE-94a. We show in the top right panel the spectrum zoomed in on Ly $\alpha$ overplotted with the best-fit model. We show the likelihood of each model parameter against all other model parameters in the other panels. The parameters plotted are (from left/top to right/bottom): expansion velocity $\left(v_{\text {exp }}\right)$, hydrogen column density $\left(n_{\mathrm{H}}\right)$, temperature $(T)$, intrinsic spread of velocities $\left(\sigma_{i}\right)$, optical depth of dust $\left(\tau_{d}\right)$, intrinsic EW $\left(E W_{i}\right)$, the systemic redshift $(z)$, and the FWHM of the observations. The best-fit parameters are indicated by the blue lines in each panel, while the predicted parameter ranges are shown at the top of each column. 
W. Karman et al.: Properties of low luminosity LAEs at $z>3$

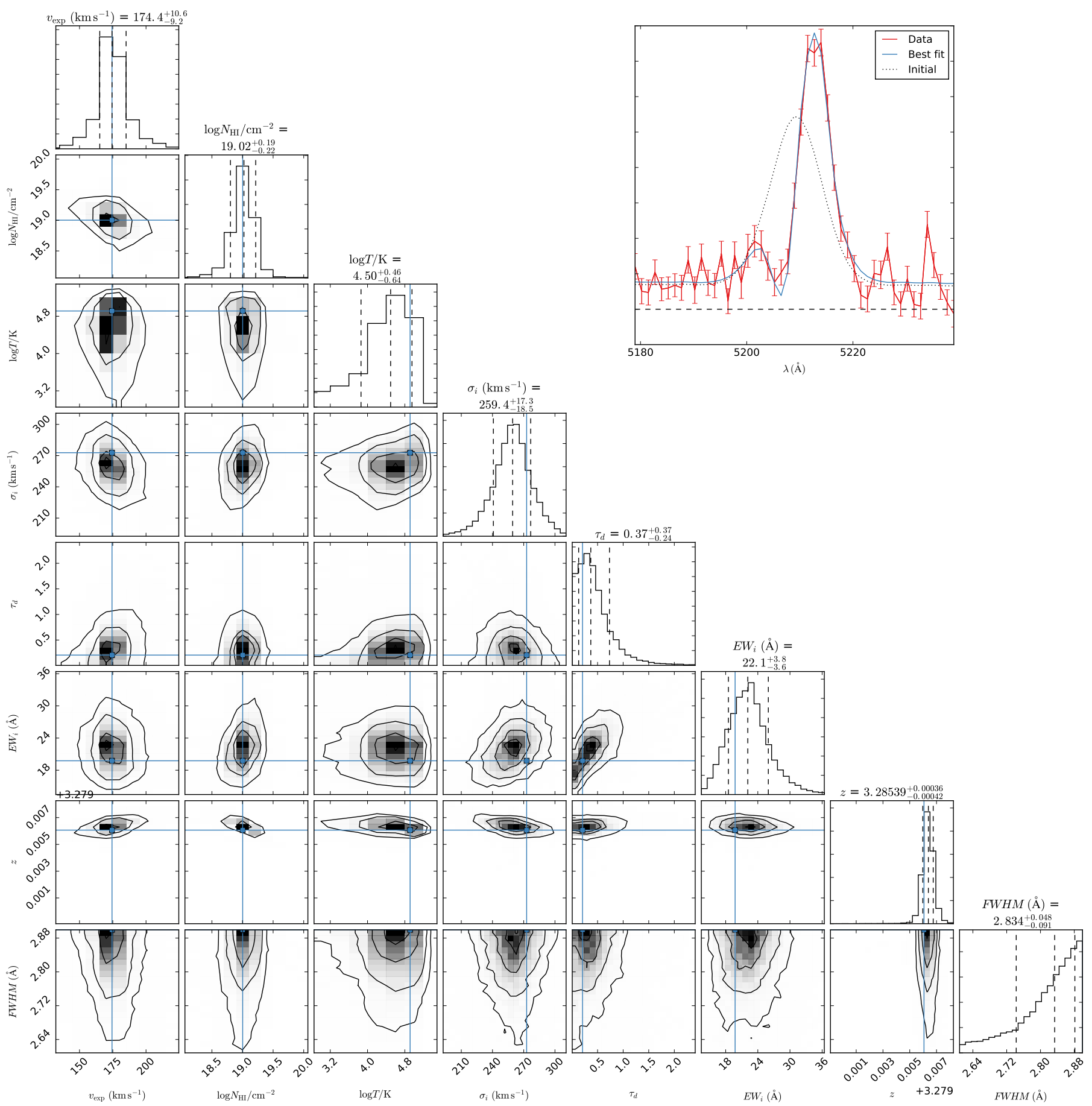

Fig. D.2. Lya modelling results for NE-94b. The lines and legend are the same as in Fig. D.1. 


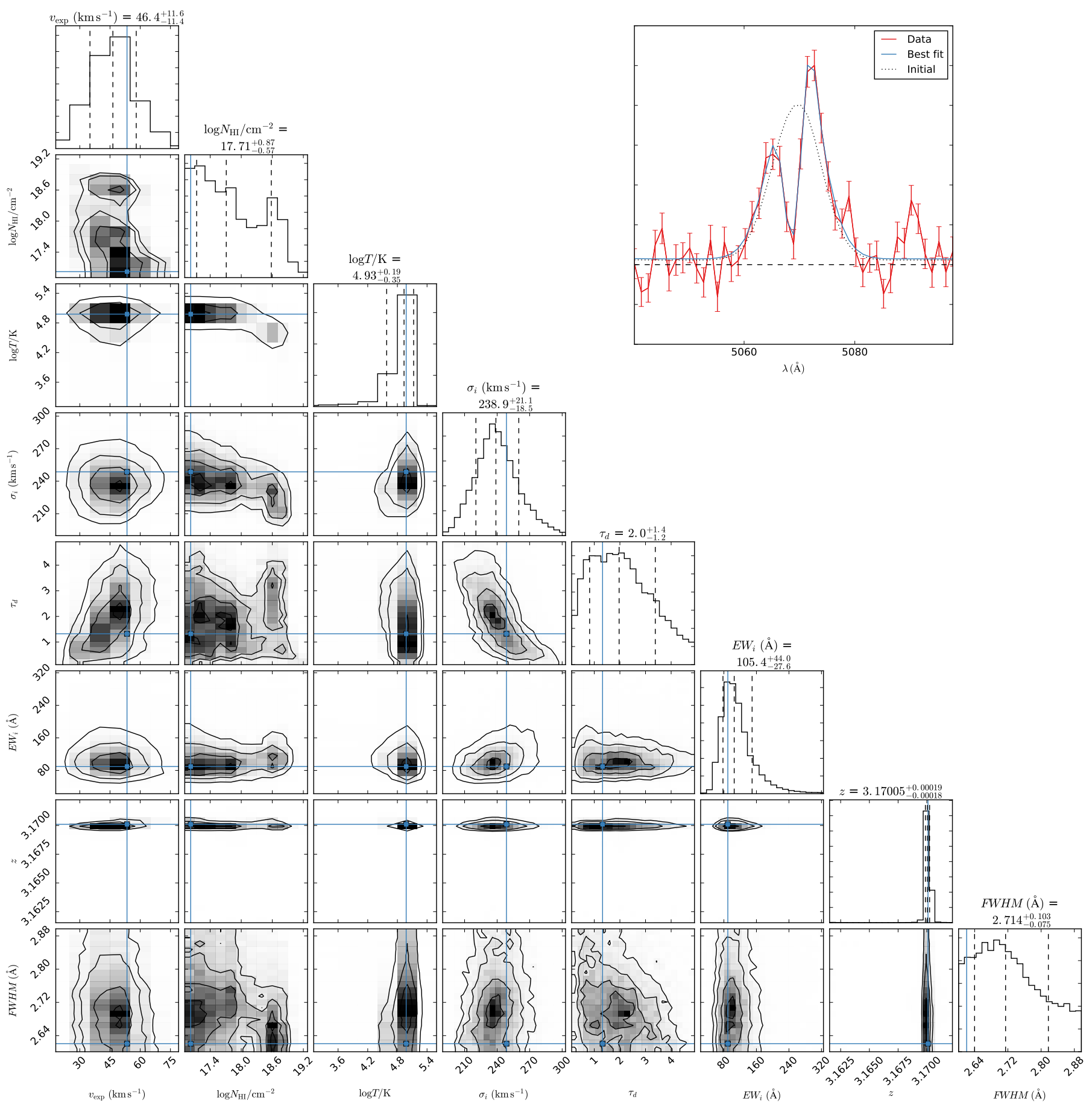

Fig. D.3. Lya modelling results for NE-93b. The lines and legend are the same as in Fig. D.1. 
W. Karman et al.: Properties of low luminosity LAEs at $z>3$
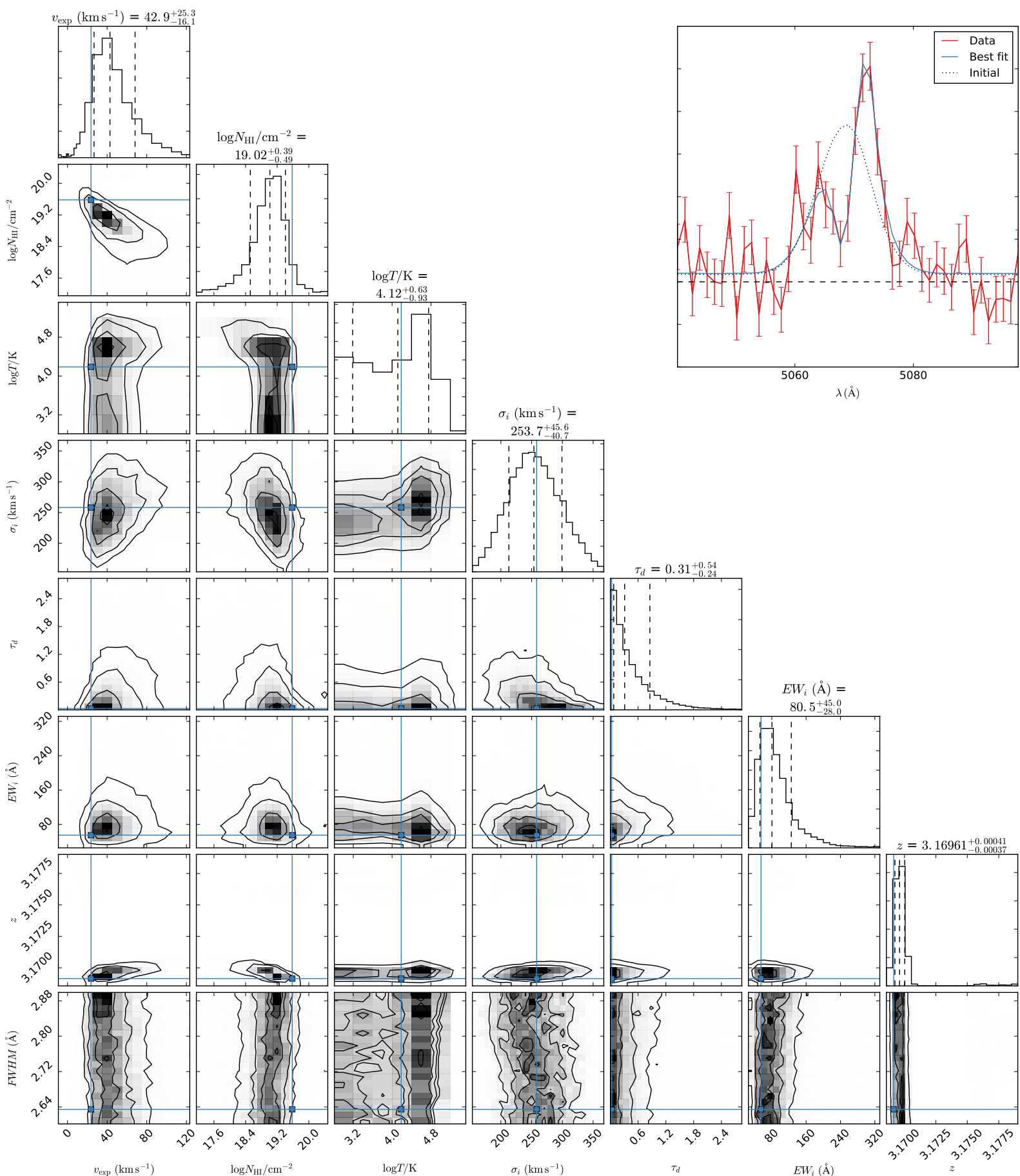

$E W_{i}(\AA)=$ $80.5_{-28.0}^{+45.0}$

Fig. D.4. Lya modelling results for NE-93a. The lines and legend are the same as in Fig. D.1. 


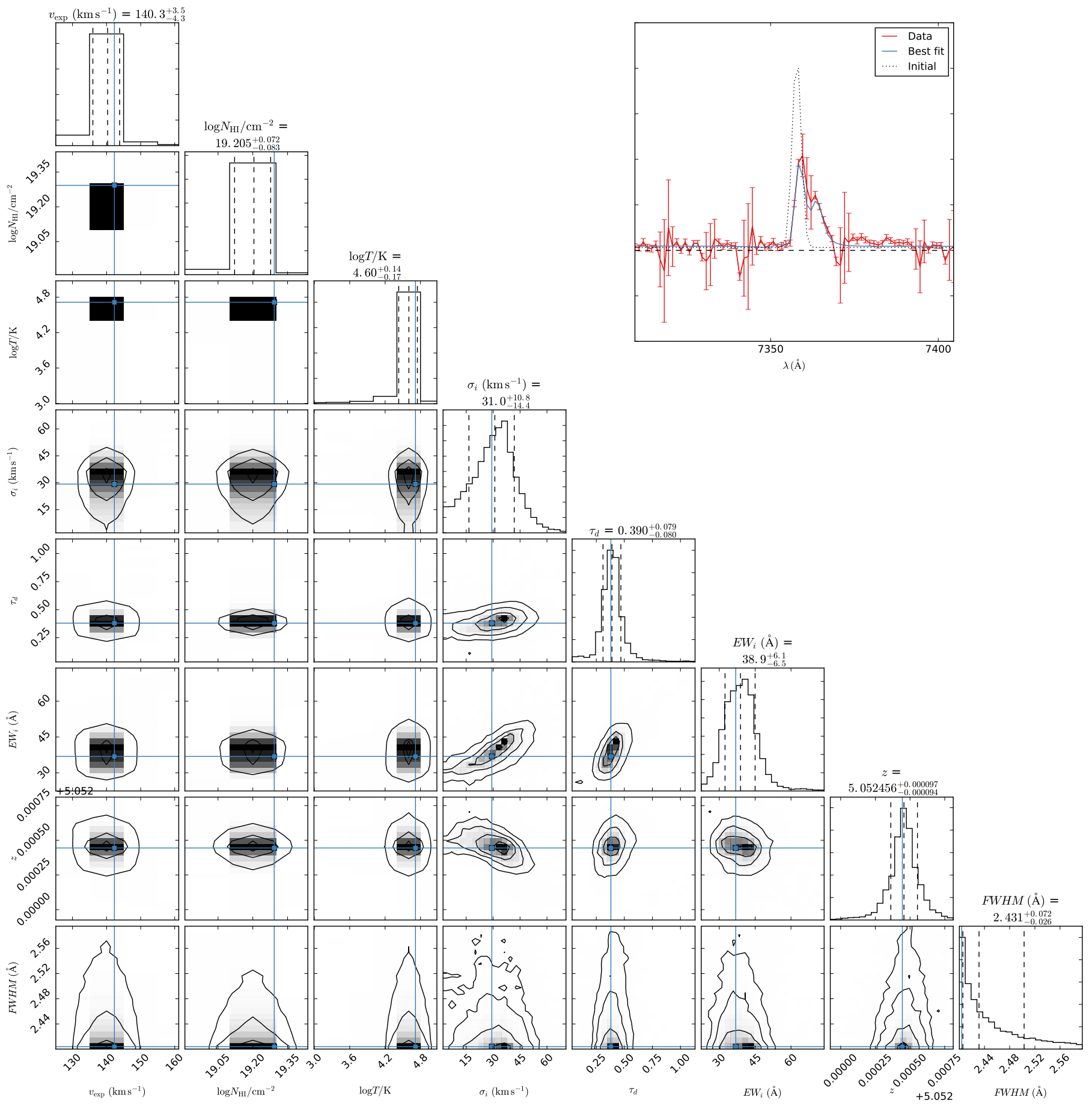

Fig. D.5. Lya modelling results for NE-98a. The lines and legend are the same as in Fig. D.1. 
W. Karman et al.: Properties of low luminosity LAEs at $z>3$

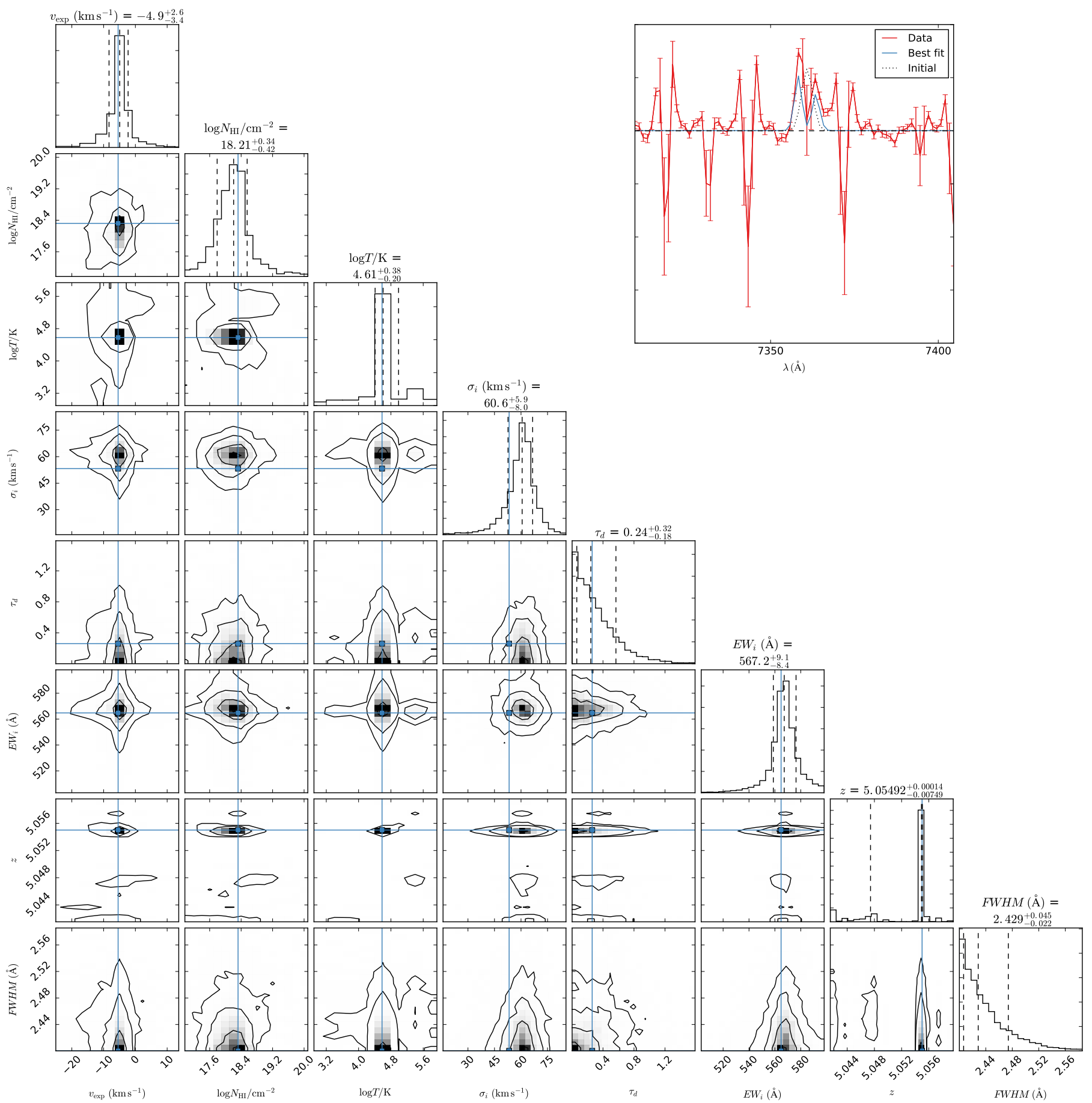

Fig. D.6. Lya modelling results for NE-98b. The lines and legend are the same as in Fig. D.1. 


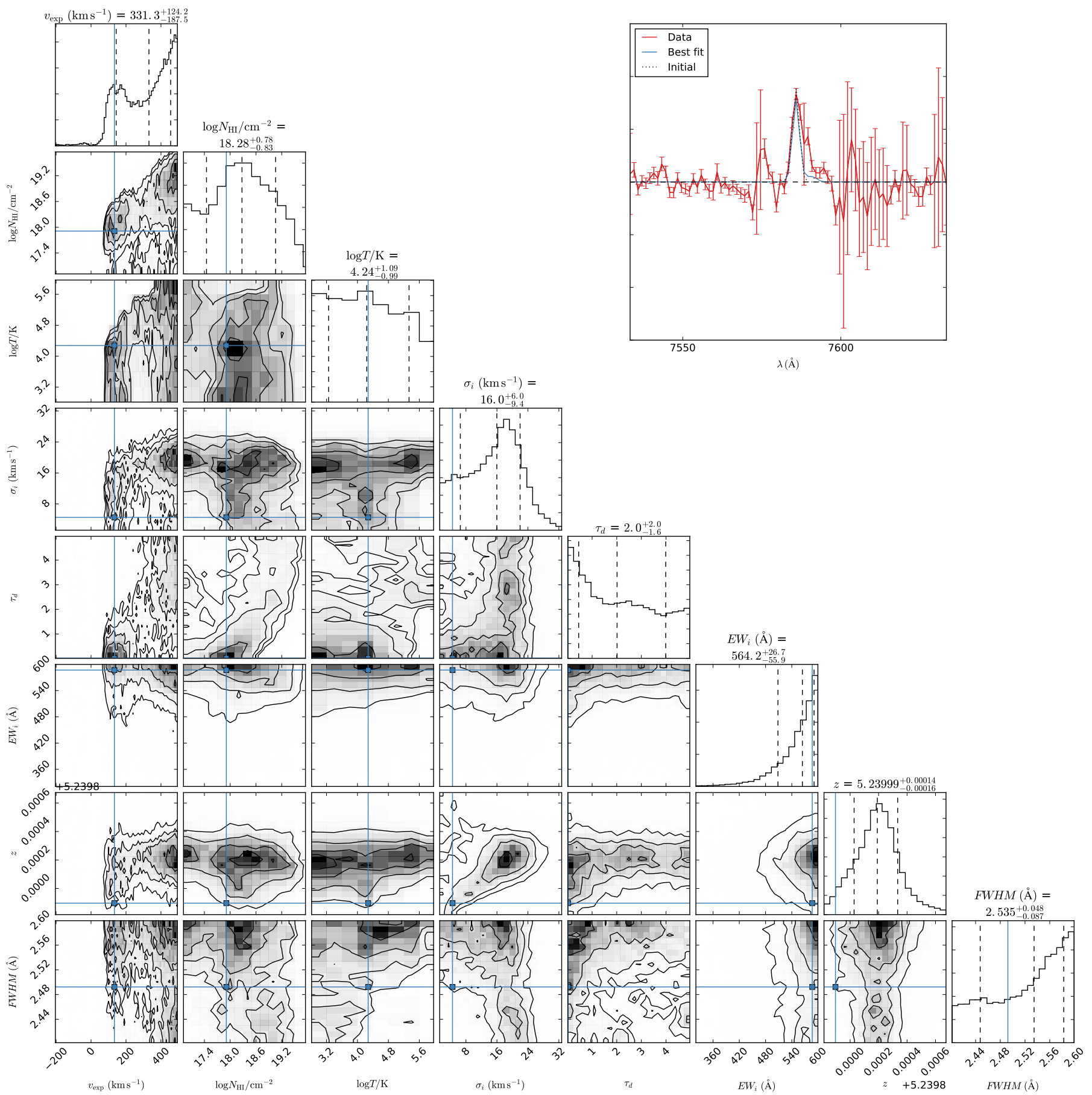

Fig. D.7. Lya modelling results for NE-99a.The lines and legend are the same as in Fig. D.1. 
W. Karman et al.: Properties of low luminosity LAEs at $z>3$

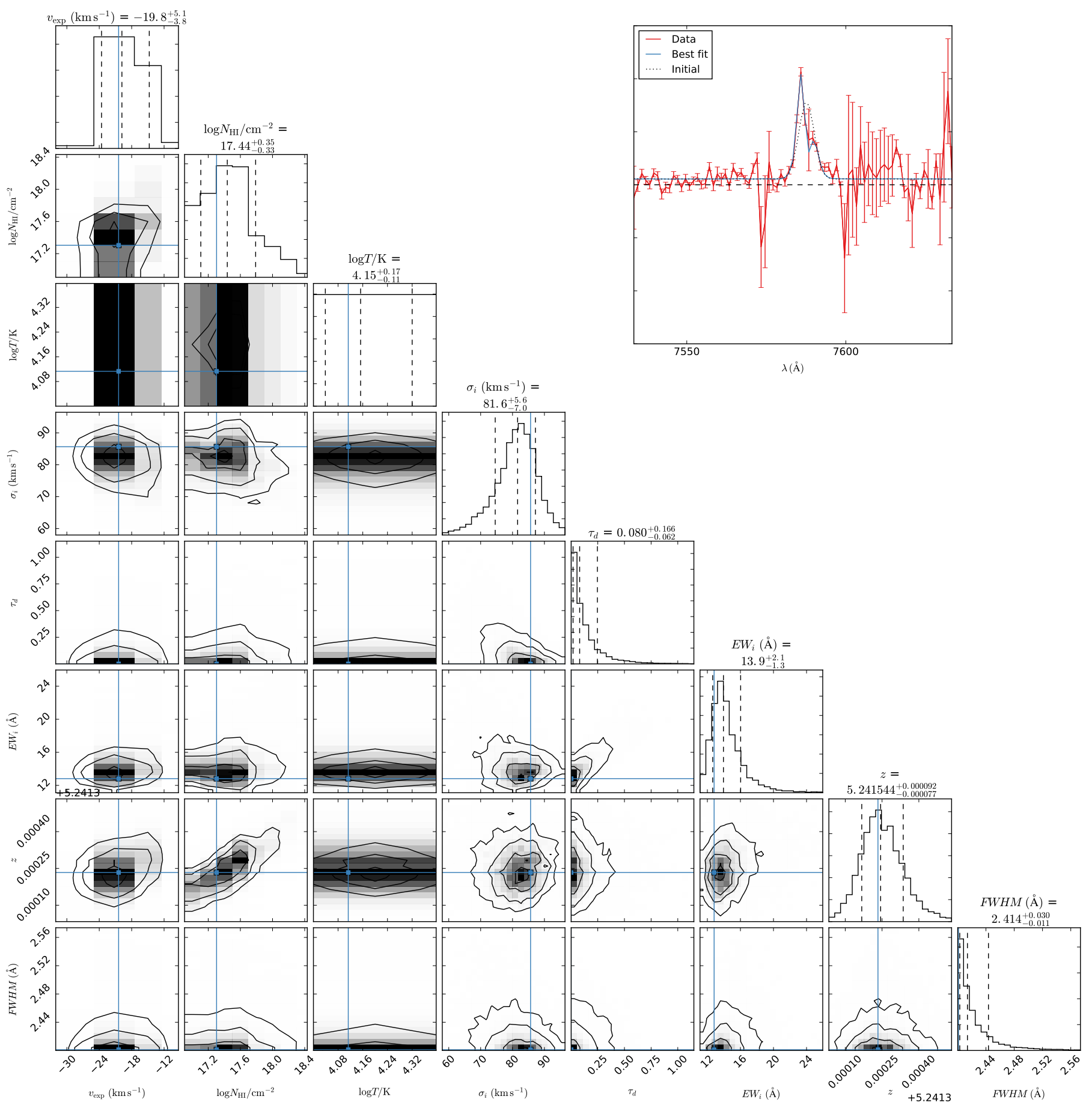

Fig. D.8. Lya modelling results for NE-99b. The lines and legend are the same as in Fig. D.1. 


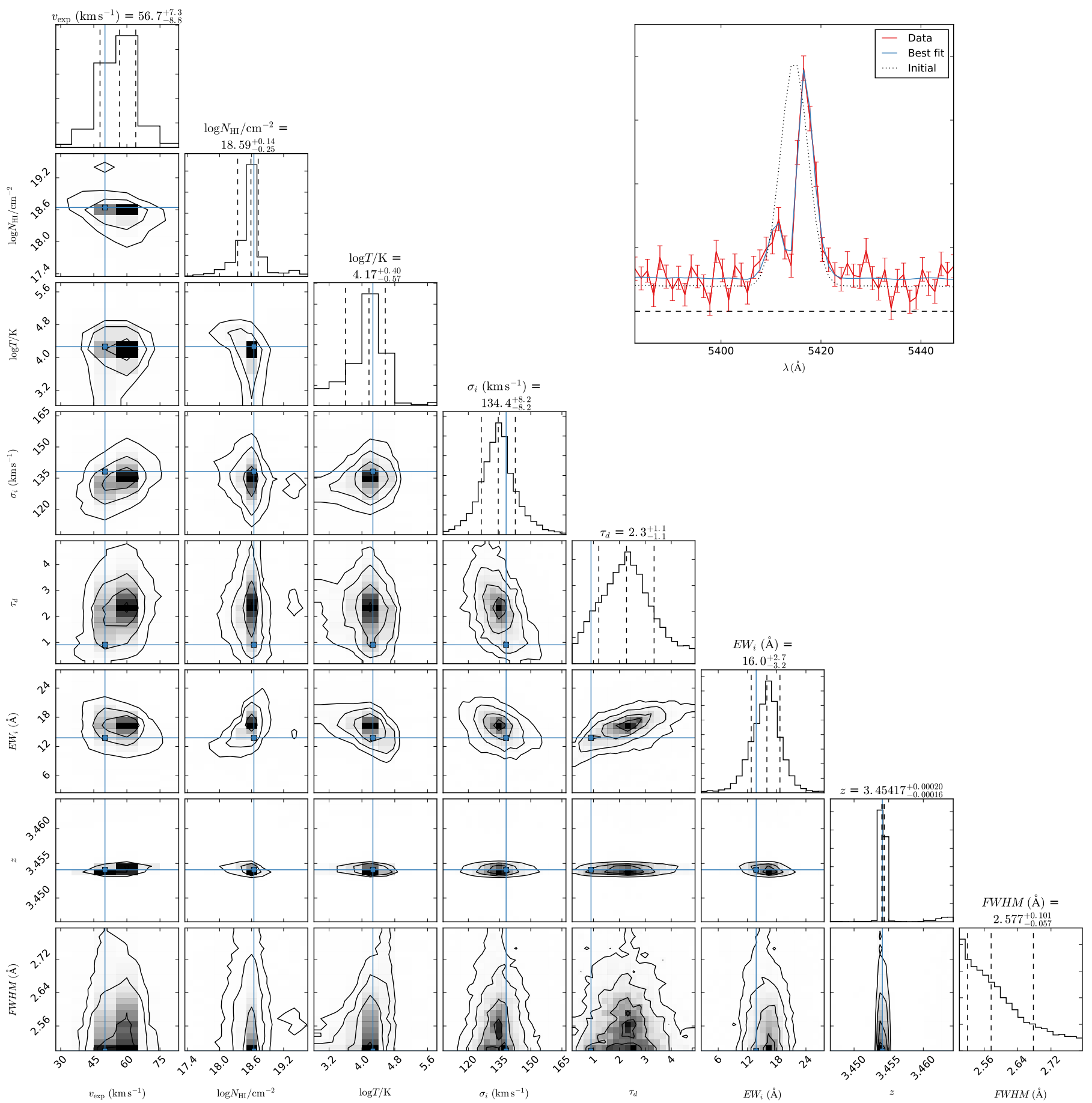

Fig. D.9. Lya modelling results for NE-96. The lines and legend are the same as in Fig. D.1. 
W. Karman et al.: Properties of low luminosity LAEs at $z>3$

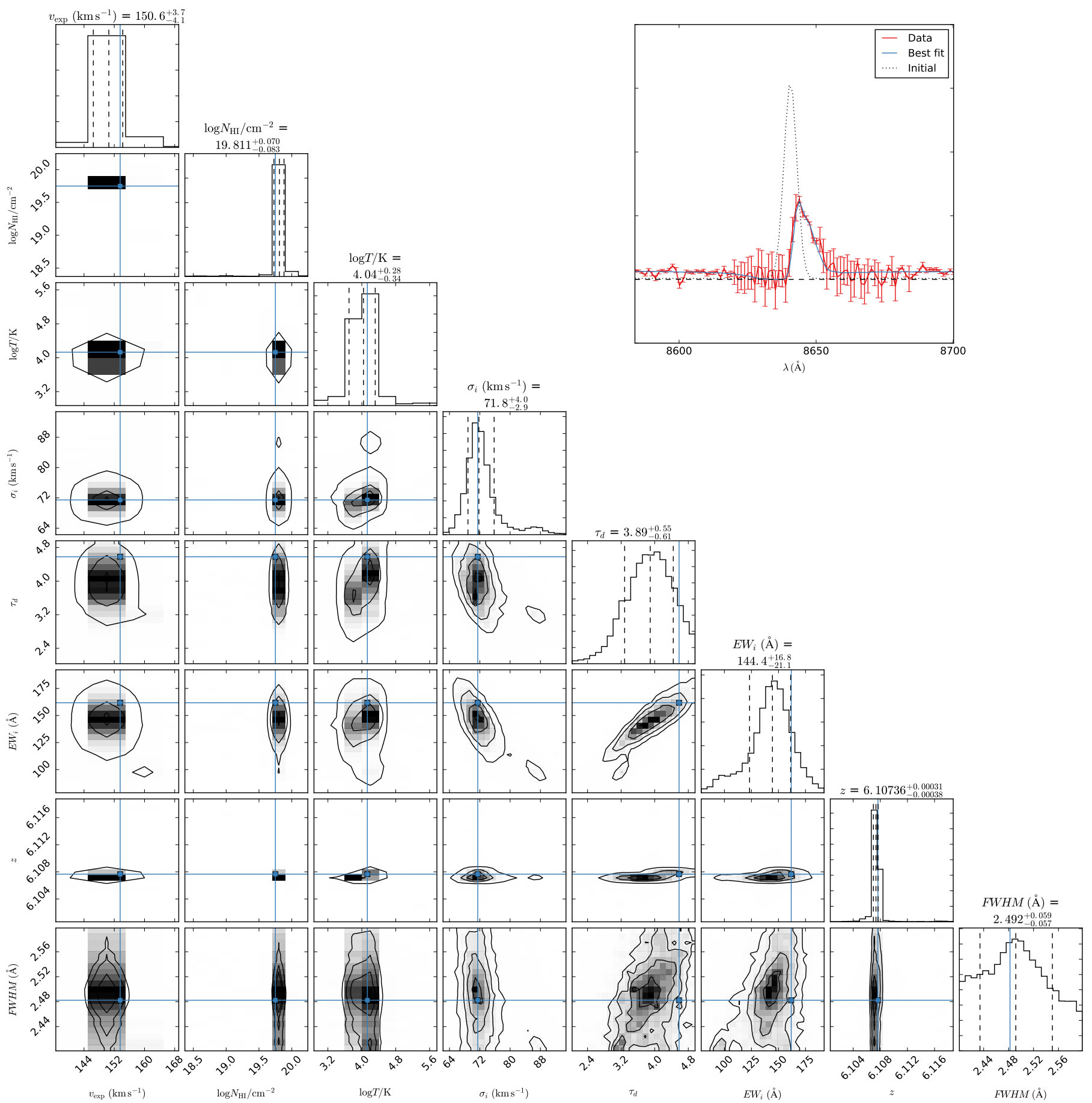

Fig. D.10. Lya modelling results for SW-53a. The lines and legend are the same as in Fig. D.1. 


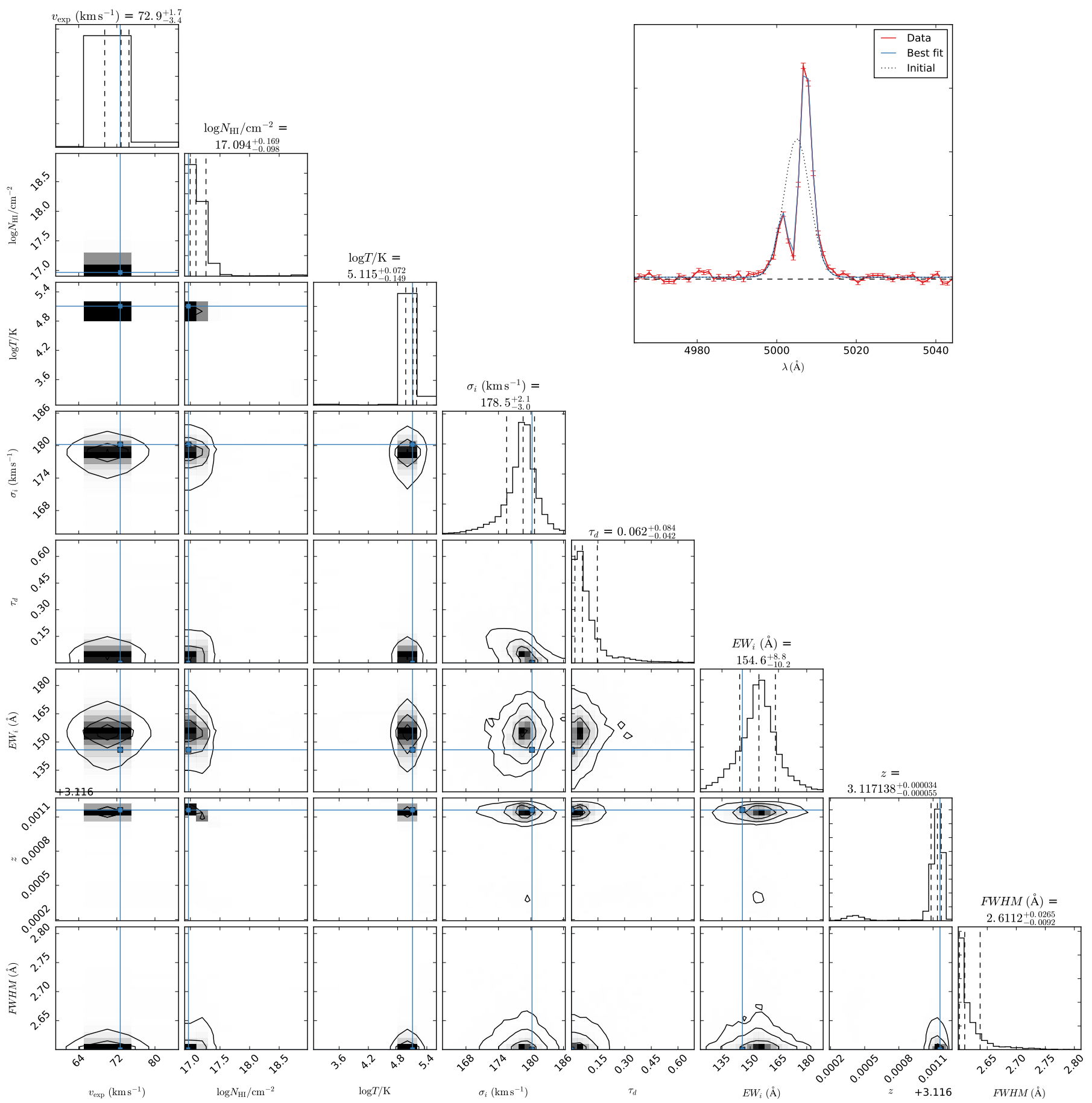

Fig. D.11. Lya modelling results for SW-49a. The lines and legend are the same as in Fig. D.1. 
W. Karman et al.: Properties of low luminosity LAEs at $z>3$

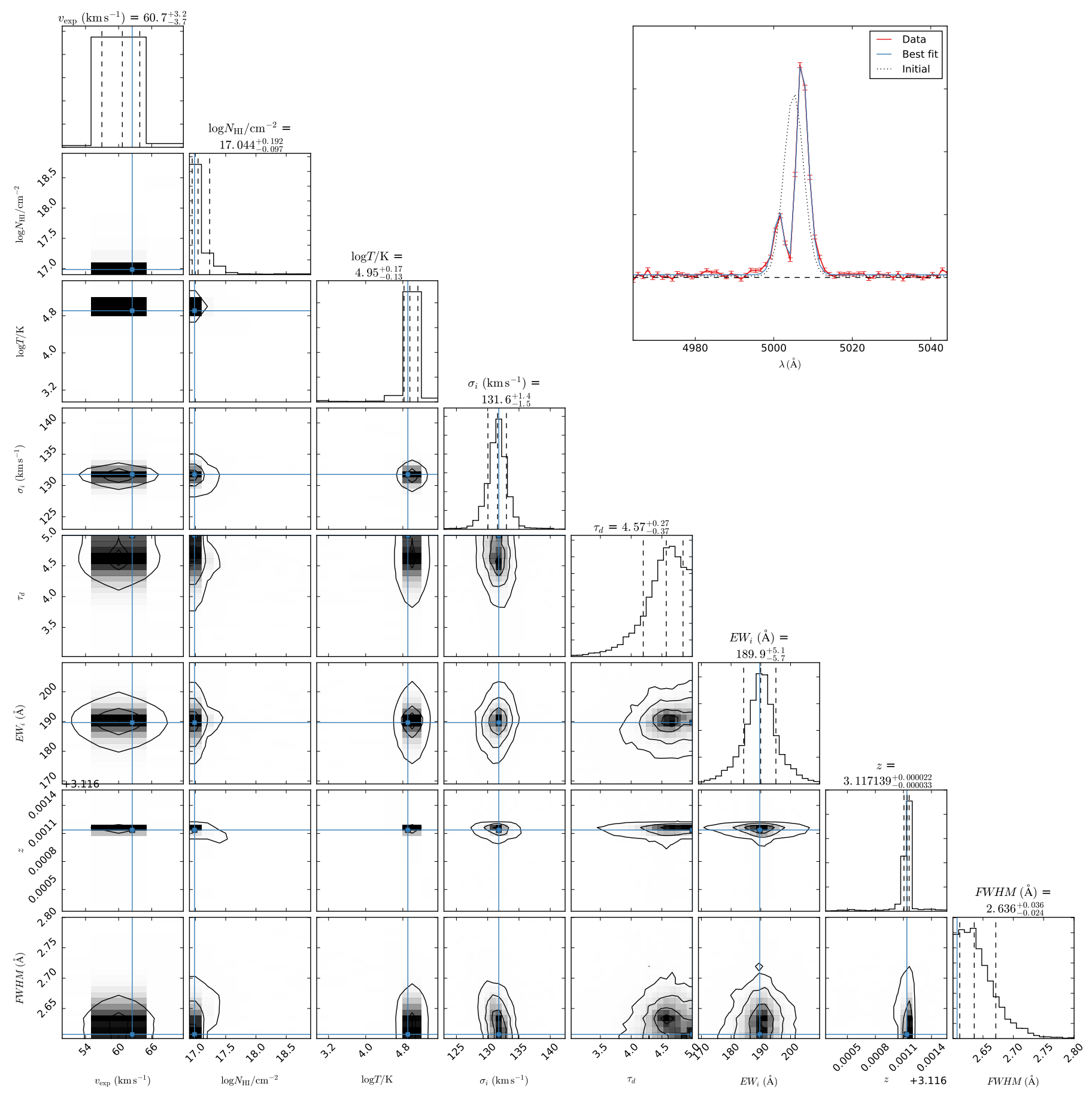

Fig. D.12. Lya modelling results for SW-49b. The lines and legend are the same as in Fig. D.1. 


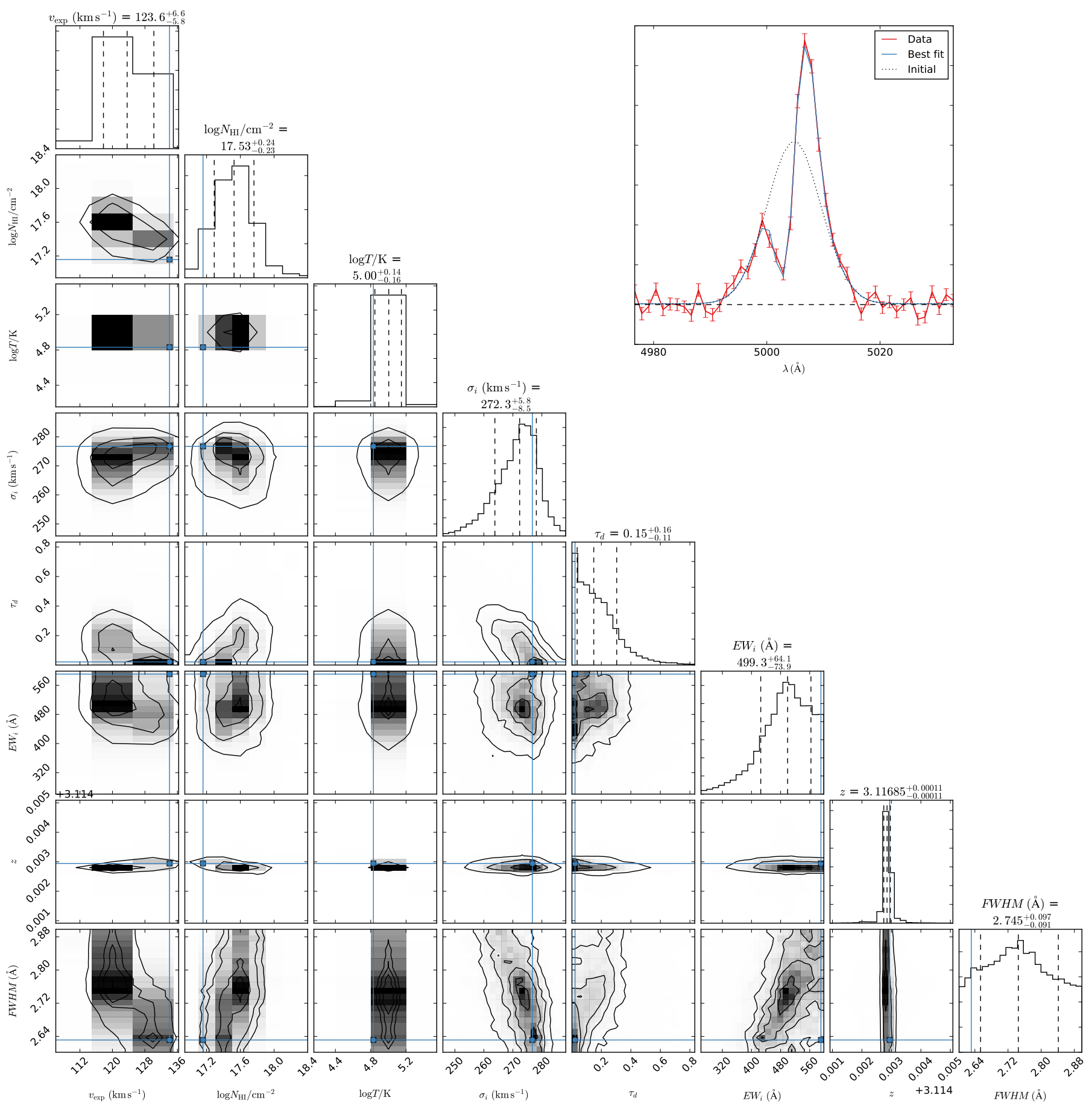

Fig. D.13. Lya modelling results for SW-50. The lines and legend are the same as in Fig. D.1. 
W. Karman et al.: Properties of low luminosity LAEs at $z>3$

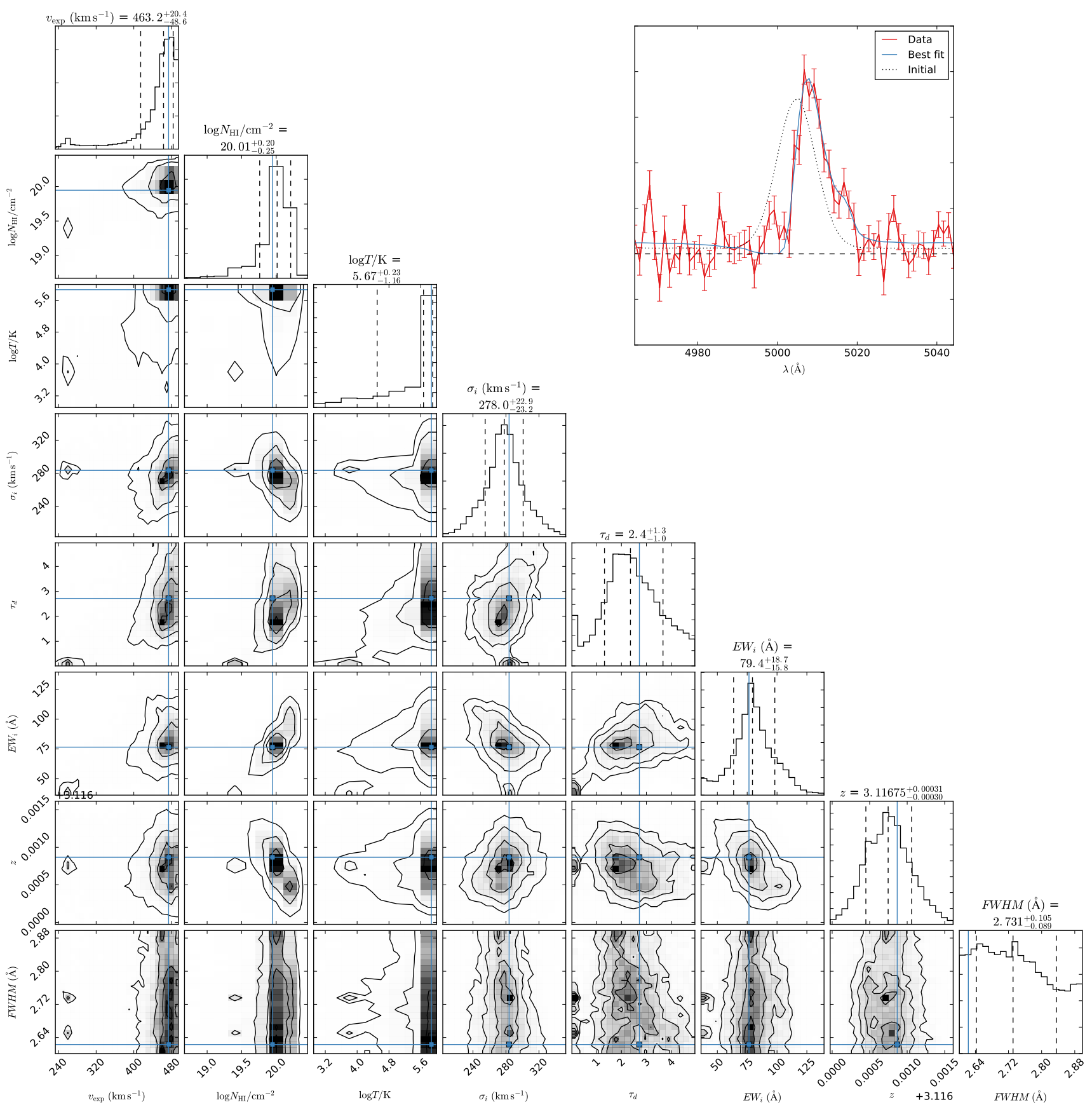

Fig. D.14. Lya modelling results for SW-68a. The lines and legend are the same as in Fig. D.1. 


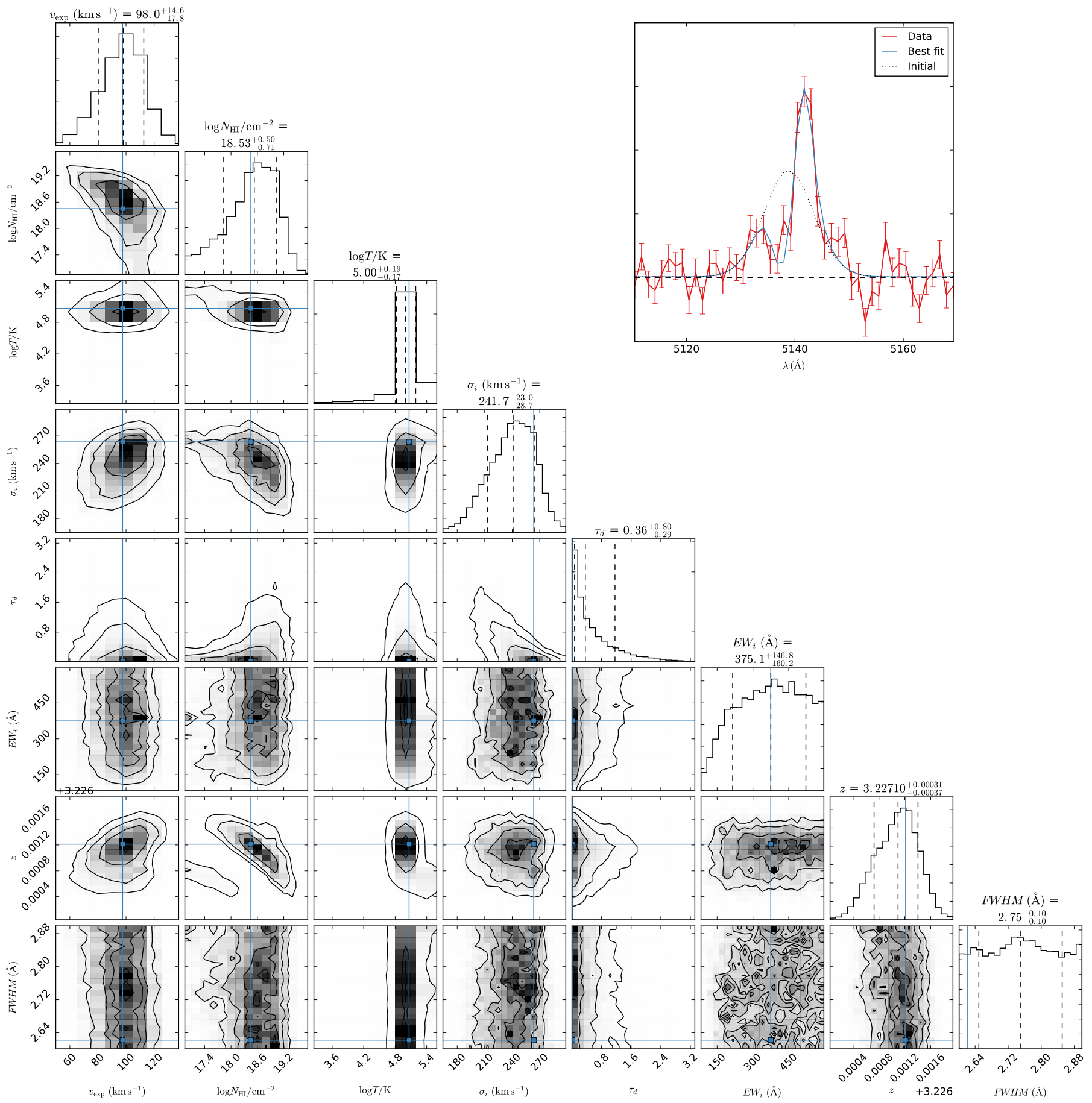

Fig. D.15. Lya modelling results for SW-51. The lines and legend are the same as in Fig. D.1. 
W. Karman et al.: Properties of low luminosity LAEs at $z>3$

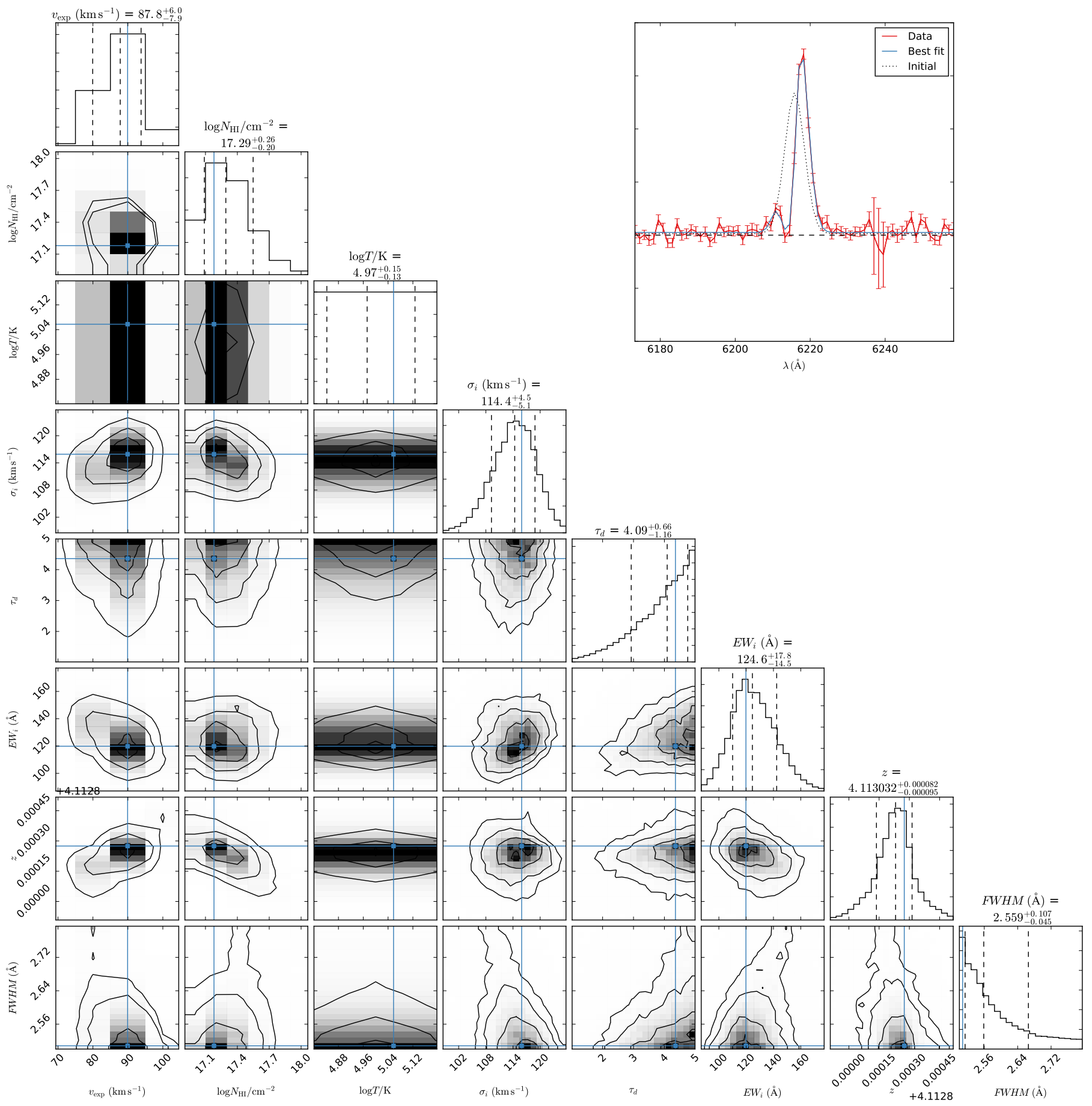

Fig. D.16. Lya modelling results for SW-52a. The lines and legend are the same as in Fig. D.1. 


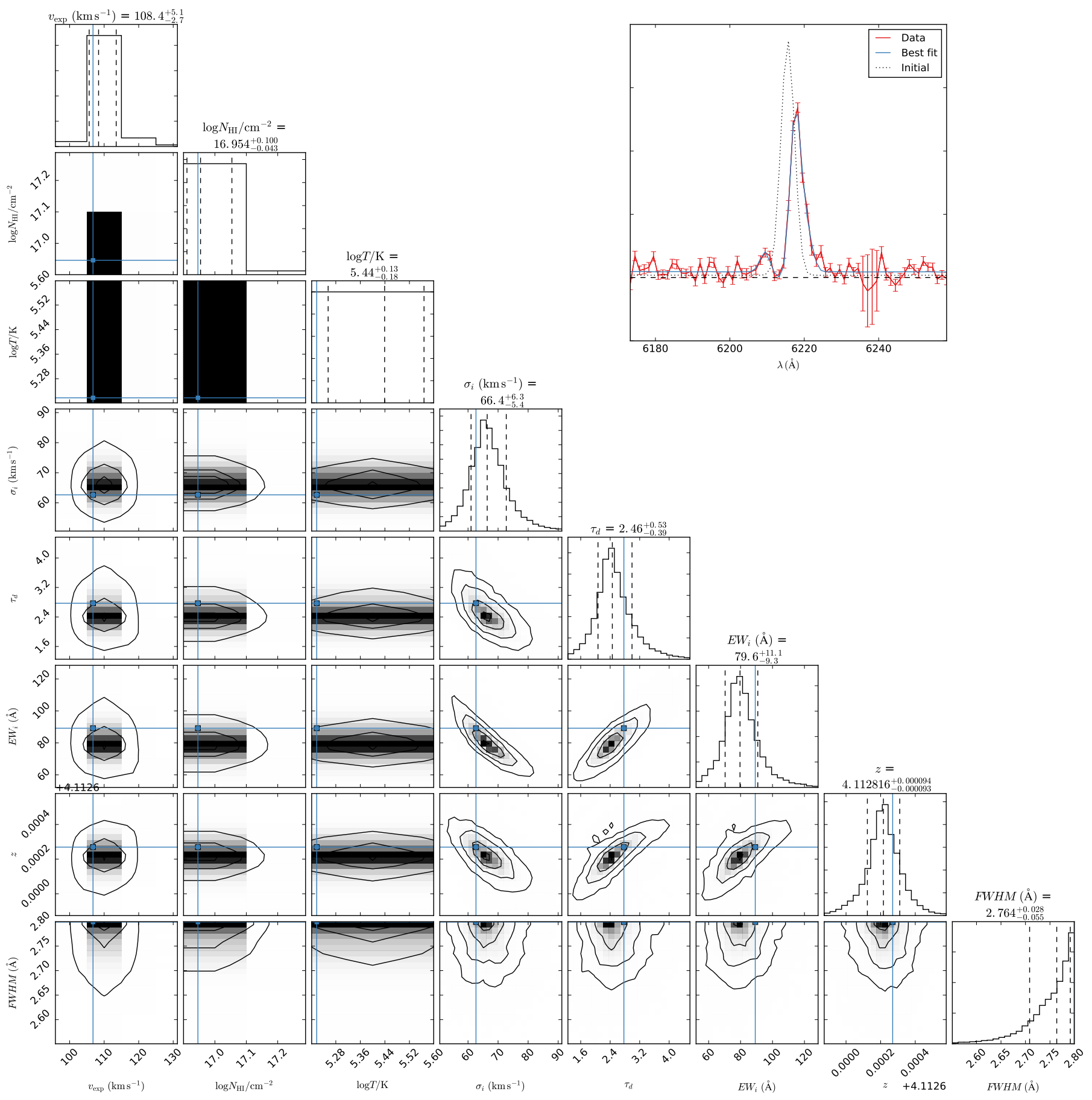

Fig. D.17. Lya modelling results for SW-52b. The lines and legend are the same as in Fig. D.1. 
W. Karman et al.: Properties of low luminosity LAEs at $z>3$

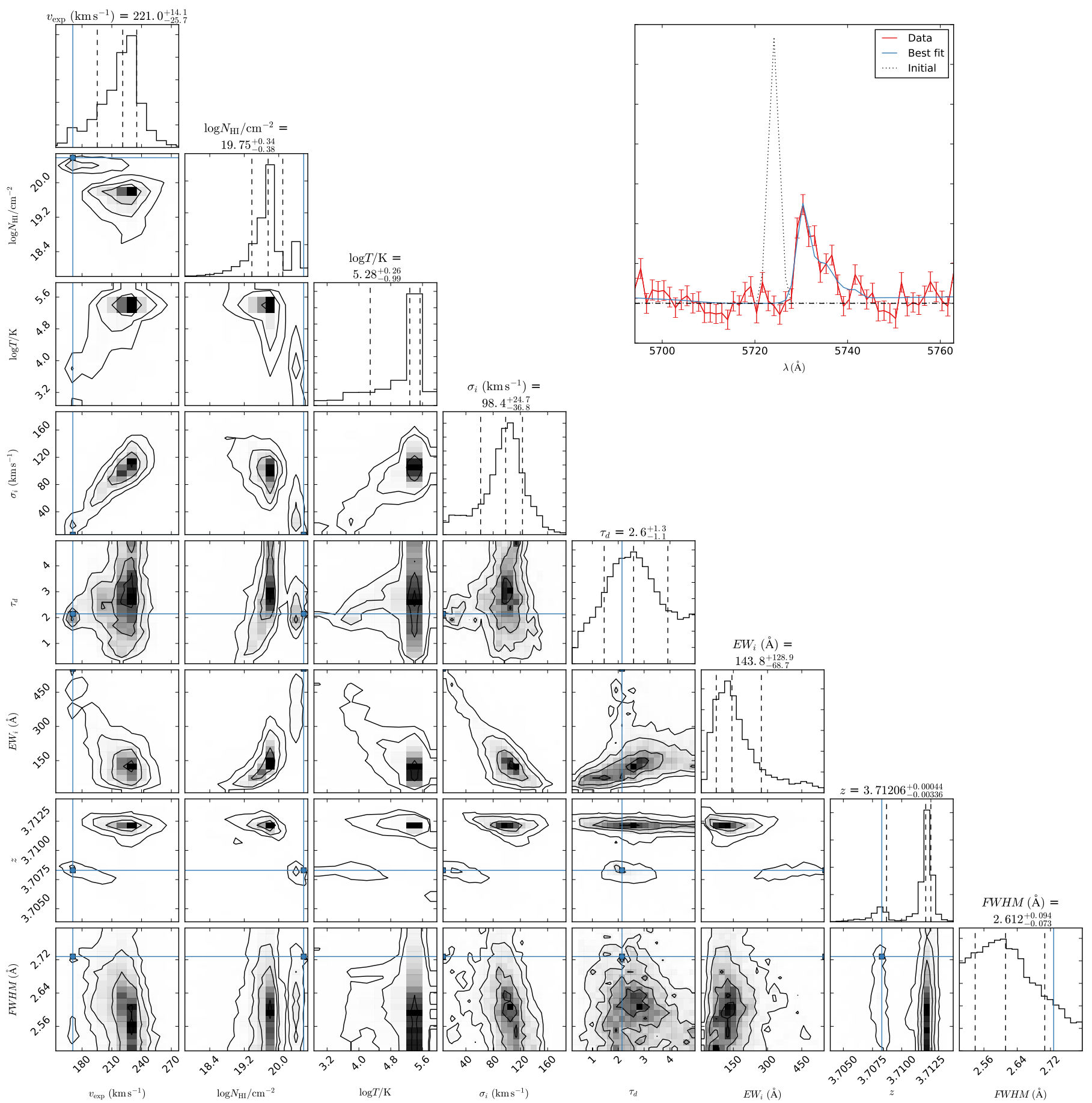

Fig. D.18. Lya modelling results for NE-97.The lines and legend are the same as in Fig. D.1. 